\title{
2 Vom Erkunden und Verirren: THE ELDER SCROLLS V: SKYRIM Und THE BANNER SAGA
}

\section{Genrepoetik versus Genreproduktion}

Die mythopoetische Welt, die Eukatastrophe, Gut und Böse als Baustoff, schließlich und vor allem die Sehnsucht nach dem ganz Anderen als das ästhetische Gefühl, das die Fantasy zu erzeugen strebt - gehen wir davon aus, diese Bestandteile einer Genrepoetik seien nicht willkürlich zusammengeklaubt, sondern nähmen ihren Platz mit einigem Recht ein. Dann stellt sich dennoch die Frage nach der Stabilität des ganzen Konstrukts. Vorhin sagte ich, meine Überlegungen würden weder auf eine normative Einhegung der Fantasy abzielen, noch den Versuch darstellen, in deskriptiver Weise das Gegebene zu erfassen. Vielmehr seien sie als Beitrag gedacht, das Potenzial des Genres aufzuschließen. Man könnte auch sagen: Sie wollen eine Idee davon skizzieren, was Fantasy sein könnte.

Wie aber ergeht es dieser Idee auf ihrem Weg in die Niederungen des Alltags? Was bleibt von ihr in den Produktionsmühlen des Kunstbetriebs? Nicht allzu viel, fürchte ich. Aus meiner Sicht gibt es kein zweites Genre, bei dem ein so weiter Abgrund klafft zwischen dem, was es ist, und dem, was es sein könnte. Das heißt natürlich nicht, dass keine großartigen Fantasyromane geschrieben (oder Fantasyfilme und -serien gedreht) werden würden. Doch merkwürdigerweise scheinen viele Freunde des Genres eine Neigung mit seinen Verächtern zu teilen - namentlich jene, die Fantasy bevorzugt von ihrer infantilen Seite zu betrachten. Die einen mögen feiern, was die anderen für albern, läppisch oder gar schädlich erachten; dem Genre jedenfalls tut diese allseitige Anspruchslosigkeit nicht gut.

Brian Attebery hat bereits zu Beginn der achtziger Jahre festgestellt, dass es ein Tolkien-Epigonentum gibt, das bestenfalls die Verballhornung des Vorbilds erzielt, häufig genug jedoch eine Art künstlerische Negation herbeiführt. Im Folgenden sei eine längere Passage aus Atteberys Polemik zitiert:

The Lord of the Rings was, as Roger Sale says, fearfully abused by those who saw it only as an avenue of escape and were blind to its strengths and weaknesses as a work of art. That is not an extraliterary experience but a subliterary one. And what kind of influence results from a source which was not adequately comprehended in the first place? Undigested meals produce nightmares, or ought to.

At any rate, undigested Tolkien has produced some literary nightmares of the first order. The worst offender to date is probably Terry Brooks's The Sword of Shannara. With all respect to the author, who may have intended homage to Tolkien, this book and others 
like it are nothing more than throwbacks to the bad old days of „The Culprit Fay“. They attempt to evoke wonder without engaging the mind or emotions, and they threaten to reduce Tolkien's artistic accomplishment to a bare formula. One finds in them nothing that was not in their model - Shannara is especially blatant in its point-for-point correspondence - but one most certainly does not find everything that was in the original. The solid homeyness of the Shire, the eerie aliveness of the forests, the brooding evil of Mordor, the despairing heroism of Frodo: all of these are lost, because they were rooted in Tolkien's own life and philosophy. His gold, like the fairies', turns to trash when it is stolen away.

To attempt to copy Tolkien is necessarily to misread, to mistake the mechanics of his tale for the substance. Roger Sale makes a good case for The Lord of the Rings as a study of "modern heroism“, very much the product of two world wars and the upheavals of English urbanization. Elves and rings and hobbits are part of Tolkien's way of confronting his life. But one finds no confrontation in the Tolkien imitations, only an engineered plot with characters that are unmotivated, unmemorable, and, God help us, cute. ${ }^{1}$

Nun geht es mir nicht um die Bewertung von Terry Brooks' Werk; und ich bin mir auch nicht sicher, ob Attebery seiner Sache einen Gefallen tut, wenn er die unnachahmliche Einmaligkeit Tolkiens in den Mittelpunkt der Argumentation stellt. Dessen ungeachtet trifft seine Kritik an der epigonalen Fantasy etwas Wesentliches. Bis heute - und das gilt selbstverständlich nicht nur für die US-amerikanische Tradition, von der Attebery spricht - gibt sich das Gros der Genreproduktionen damit zufrieden, einer Formelhaftigkeit zu frönen, die sich in der Repetition stereotyper Handlungsfigurationen und dem Ausstanzen von Figurenhülsen erschöpft. Das ist dann gewissermaßen die künstlerische Entsprechung zur taxonomischen Genretheorie: eine zwangsweise Stillstellung der Dynamik, die in der Fantasy walten könnte.

Zweifellos haben wir es hier mit einer Maschinerie zu tun, welche die kommerzielle Verwertung der Kunst überhaupt (und keineswegs nur die sogenannte Unterhaltungsliteratur) antreibt. Doch wenn es um Fantasy geht, sind die Erzeugnisse dieser Maschinerie besonders mängelbehaftet. Das hat zum einen damit zu tun, dass es zur Gestaltung einer tauglichen Sekundärschöpfung, wie Tolkien sagt, „vermutlich einiger Mühe und Überlegung“ braucht. Was ihn betrifft, nahmen das Sich-Mühen und Überlegen bekanntlich Jahrzehnte in Anspruch, und obgleich gewiss nicht jede Fantasywelt so elaboriert sein muss wie Mittelerde, kann man vermuten, dass die Schnelllebigkeit des Literaturbetriebs tendenziell der geduldigen Ausarbeitung einer Sekundärschöpfung entgegensteht.

1 Brian Attebery: The Fantasy Tradition in American Literature. From Irving to Le Guin. Bloomington 1980, S. 155-156 [Herv. i. O.]. 
Viel wichtiger aber ist, dass die Fantasy, wie bereits erwähnt, einer Idee von Gut und Böse bedarf, die die Konstruktion des jeweiligen Werks bis in kompositorische Details hinein prägt. Ein Dunkler Herrscher, der die Welt unterjochen oder vernichten will, so wie sich andere Leute aus Langeweile vor den Fernseher setzen, ist und bleibt eine Schießbudenfigur (außer natürlich, die Leere und Motivationslosigkeit des Bösen wären selbst als metaphysisches Grauen gestaltet), und ein Heroismus, der sich in besonders effektivem Schwerterschwung erschöpft, läuft bestenfalls auf peinliche Kraftmeierei, schlimmstenfalls (und da hat Arno Meteling zweifellos recht) auf protofaschistisches Herrenmenschentum hinaus. Kurzum, was immer die Überzeugungen der Autorinnen und Autoren sein mögen - ihre Werke müssen an etwas glauben. Wobei es hier nicht um ein Ideal von Erbauungsliteratur geht: Auch der Nihilismus kann ein Glaube sein. Eine Kunst, die in diesem Sinn erfüllt oder inspiriert ist, wird aber nicht aus der poetologischen Entkernung entstehen, die mit der Reproduktion von Plot-Mechaniken einhergeht, sondern verlangt im Gegenteil nach der Durchdringung des poetologischen Kerns eines Werkes, wenn selbiges zum Vorbild des eigenen Schaffens werden soll.

Man kann sich des Problems auch nicht dadurch entledigen, dass man Tolkiens Einfluss kleiner zu reden sucht, als er sehr wahrscheinlich ist. Denn zum einen stellt The Lord of the Rings ja nicht die letztgültige Enthüllung der Fantasy dar, sondern bildet eher einen Kristallisationspunkt der verschiedenen ästhetischen Strömungen, aus denen sich das Genre speist; einen Kristallisationspunkt, der insofern auch Höhepunkt ist, als seine konkrete Ausgestaltung und Wirkmacht sicherlich - so gesehen ist Attebery zuzustimmen - durch eine in dieser Form unwiederholbare historische und personale Konstellation ermöglicht wurden. Zum anderen bleibt, wenn die von mir skizzierte Genrepoetik das Entscheidende trifft, das Problem mutatis mutandis auch dann bestehen, wenn nicht Tolkien, sondern Robert E. Howard, Ursula K. Le Guin, Markus Heitz oder irgendjemand sonst für das exemplarische Werk verantwortlich zeichnet. Und noch, wenn überhaupt kein Autor, keine Autorin, sondern ein Film, eine Fernsehserie, ein Comic oder ein Rollenspielsystem die Inspirationsquelle abgibt, ist die Herausforderung dieselbe; stets gilt es, die Nachahmung und Weiterführung aus dem Innersten der jeweiligen Poetik - beziehungsweise der jeweiligen Poetiken - heraus zu entwickeln; sei es in affirmierender oder ablehnender Weise. ${ }^{2}$

2 Steven Erikson etwa, der vor allem für die zehnbändige Romanserie Malazan Book of the Fallen (1999-2011) bekannt ist, betrachtet sowohl Tolkiens Werk als auch offensichtlich auf The Lord of the Rings basierende Rollenspielsysteme wie Advanced Dungeons \& Dragons (1977-1979, entwickelt von Gary Gygax) vornehmlich als ein Set aus Regeln und Stereotypen, auf die man sich bezieht, um sie dann gegen sich selbst zu wenden oder vollständig aufzulösen. Freilich 
Ich meine, dass dieses Ansinnen so oft scheitert, weil die Fantasy ihren Leumund insofern Lügen straft, als sie im Grunde ein überaus anspruchsvolles und komplexes Genre ist. Es mag seine Logik haben, dass der Anspruch und die Komplexität in sich zusammenfallen, bis tatsächlich nur abgeschmackter Unfug zurückbleibt, wenn die ästhetische Konstruktion nicht auf dem Fundament politischer, ethischer, philosophischer oder religiöser Überzeugungen ruht, die zwar das Genre durchwalten, ohne jedoch in ihm ihren Ursprung zu haben.

\section{Fantasy und Videospiel: eine privilegierte Beziehung}

Allerdings gibt es ein Medium, in dem sich die Fantasy verblüffend häufig mit großem künstlerischen Erfolg realisiert: das Videospiel. ${ }^{3}$ Tatsächlich erstaunt es, wie viele Titel, die als Meilensteine in der Entwicklung des Mediums gelten, diesem Genre zuzurechnen sind. ${ }^{4}$ Das reicht (mindestens) zurück bis zu den Anfängen des Videospiels als kommerzielle Kunstform für den Hausgebrauch: ULTIMA (Origin Systems, 1981), DRAGON'S LAIR (Cinematronics, 1983), THE BARD'S TALE (Interplay Productions, 1985), THE LEGEND OF ZELDA (Nintendo EAD, 1986) und FINAL FANTASY (Square, 1987) sind hier einige der einflussreichsten Titel, die wohl jedem, der sich eingehender mit dem Medium beschäftigt hat, wenigstens vom Hörensagen her bekannt sind. ${ }^{5}$ Zumal sämtliche genannten Spiele

leugnet er nicht, dass diese Art von negativistischer Bezugnahme immer noch einhergeht mit der Anerkennung einer Tradition: „So, I can rail at the clichés established by AD\&D, but man, they're in my fucking blood, like it or not. I use them. All. The. Time. And lo, it's not a problem. In fact, I depend on them: as my readers know, in the Malazan series there's scant else for them to connect with at first glance. And even as readers get a handle on them, I mess them up." Vgl. Steven Erikson: The World of the Malazan Empire and Role-Playing Games. In: steven-erikson.com, http://www.steven-erikson.com/index.php/the-world-of-the-mala zan-empire-and-role-playing-games/ [letzter Zugriff: 12.05.2020].

3 Was die Verwendung dieses Begriffs betrifft, schließe ich mich Dominic Arsenault an, der da schreibt: „I use the term ,video game“ to refer to the whole of computer-processed, visually represented games, which can then further be divided in console games and computer games (a specific subset for personal computers). Others may use ,digital games" as a synonym." Dominic Arsenault: Video Game Genre, Evolution and Innovation. In: Eludamos. Journal for Computer Game Culture 3 (2009), H. 2, S. 149-176, hier: S. 175.

4 Ich werde später darauf eingehen, was „Genre“ in Hinblick auf das Videospiel bedeutet.

5 Es sei an dieser Stelle darauf hingewiesen, dass die Frage, ob Videospiele als Kunstwerke zu zählen seien, durchaus strittig ist. Daniel Martin Feige etwa ist keineswegs der Ansicht, dass alle Videospiele unter diese Kategorie fallen - und zwar unabhängig von ihrer Qualität als 
zahlreiche Fortsetzungen und Ableger hervorgebracht haben; tatsächlich setzen sich die entsprechenden Reihen - mit der Ausnahme von DRAGON'S LAIR, das sich einstweilen auf ein anderes Medium verlegt hat ${ }^{6}$ - bis in die Gegenwart hinein fort.

Mit nur leichter Übertreibung kann man von einer Umkehrung der Verhältnisse sprechen: Während die Fantasy in den Medien Film und vor allem Literatur, was die kunstkritische Rede betrifft, zu den gering geschätzten, mitunter nachgerade verachteten Genres zählt, wird sie allseitig gefeiert, sowie es um Videospiele geht. Wie lässt sich das erklären? Es greift wohl zu kurz, auf den Konservativismus des feuilletonistischen Mainstreams zu verweisen, der durch die bedauerliche Neigung gekennzeichnet ist, ästhetische Formen $\mathrm{zu}$ missachten, in denen sich eine Bedrohung der eigenen Deutungshoheit ausdrücken könnte. ${ }^{7}$ Vielmehr muss diese Umkehrung auf intrinsische Gründe zurückgeführt werden. Das heißt: An irgendeinem Punkt geht die Poetik des Genres Fantasy eine Verbindung ein mit den besonderen Eigenschaften des Mediums Videospiel. Anders gesagt: Die Genrepoetik erstrebt etwas, das sich in diesem Medium besonders effektiv - oder wenigstens mit einer gewissen Leichtigkeit - verwirklichen lässt. Noch einmal anders: Während der Film und die Literatur dem Genre Fantasy möglicherweise

Spiele. Seiner Meinung nach ist der „Kunstcharakter“ von Videospielen darin zu erkennen, „dass wir uns im Spielen dieser Spiele selbst durchspielen; dass wir uns im Lichte des Aushandlungsgeschehens, das das Spiel ist, selbst neu aushandeln. Und dass ein Computerspiel als Kunstwerk gelingt, muss heißen, dass es etwas verhandelt, was nur in und durch die Form dieser Verhandlung zu haben ist. Die Form dieser Aushandlung heißt im Bereich des Computerspiels natürlich vor allem auch: Dass wir das Spiel spielen müssen, um es zu verstehen.“ Daniel Martin Feige: Kunst. In: ders./Sebastian Ostritsch/Markus Rautzenberg (Hg.): Philosophie des Computerspiels. Theorie - Ästhetik - Praxis. Stuttgart 2018, S. 177-192, hier: S. $186 \mathrm{f}$.

Ich weiß nicht, ob Videospiele nur unter den von Feige genannten Bedingungen als Kunst gelten können, und sicherlich ist im Einzelfall diskutabel, ob ein bestimmtes Spiel diese Bedingungen erfüllt oder nicht. Dessen ungeachtet will ich festhalten, dass alle im Verlauf dieser Studie analyiserten Spiele, wenn Feige recht hat, einen „Kunstcharakter“ aufweisen.

6 Don Bluth, einer der Erfinder von DRAGON'S LAIR, hat als Regisseur, Drehbuchautor und Produzent maßgeblichen Anteil an der Animationsfilmadaption (USA 2021), die sich auf die Geschehnisse des ersten Spiels bezieht.

7 Wer diese Behauptung für unangemessen hält, möge schauen, ob es ihm gelingt, in den großen deutschsprachigen Feuilletons - wobei ich fürchte, dass es anderswo nicht viel besser aussieht - auch nur eine Rezension eines als solchen adressierten Fantasy-Romans zu finden, die sich vorwiegend mit den literarischen Qualitäten des entsprechenden Werks auseinandersetzt. Wenn überhaupt, wird Fantasy unter soziologischen Gesichtspunkten wahrgenommen; und selbst diese Wahrnehmung ist in aller Regel auf angloamerikanische Beststeller beschränkt. 
eine Art medialer Widerständigkeit entgegensetzen, ${ }^{8}$ eignet dem Videospiel ein grundlegendes Einvernehmen mit den Erfordernissen des Genres.

Insofern besteht meines Erachtens, ich sagte es bereits, eine privilegierte Beziehung zwischen der Fantasy und dem Videospiel; wobei sich das Privileg vor allem in dem Verhältnis zwischen den Spielern und der jeweiligen Sekundärwelt ausdrückt. Es hat also etwas damit zu tun, dass das Medium die Erschließung seiner fantastischen Welten in die Hände der Spielerinnen und Spieler legt: Zuvörderst gestalten sie die audiovisuelle Rhythmik und die spezifische Zeitlichkeit dieser Erschließung. ${ }^{9}$ Mitunter ist es aber auch möglich, dass sie der Welt vermittelt über die Handlungen des Avatars ein bestimmtes politisches oder ethisches Gepräge geben; und häufig liegt es an ihnen, über die Moralität oder Amoralität des Avatars selbst zu verfügen.

8 Zumindest, wenn Michael Wedel recht hat, der davon ausgeht, dass „eine hohe Affinität zwischen dem Hollywood-Blockbuster und der Fantasy als seiner am besten geeigneten generischen oder metagenerischen Form“ besteht, woraus man ja umgekehrt den Schluss ziehen muss, dass die allermeisten Filmemacherinnen und Filmemacher kaum eine Chance haben, einen anständigen Fantasy-Film zu drehen, da sie eben nicht auf die produktionstechnischen Ressourcen zurückgreifen können, die man benötigt, um kinematografische Sekundärwelten zu erschaffen. Vgl. Michael Wedel: Das audiovisuelle Oxymoron. Spezialeffekte, Dolby Surround Sound und Fantasypoetik. In: Natascha Adamowsky/Nicola Gess/Mireille Schnyder/Hugues Marchal/Johannes Bartuschat (Hg.): Archäologie der Spezialeffekte. München 2018, S. 47-60, hier: S. 48.

Was nun die Literatur betrifft, so besteht die mediale Widerständigkeit gegenüber der Fantasy, produktionsästhetisch gesprochen, vielleicht einfach darin, dass der Aufwand, eine in sich schlüssige Sekundärwelt zu entwickeln, für einen einzelnen Autor, eine einzelne Autorin häufig kaum zu leisten ist beziehungsweise in keinem Verhältnis zum erwartbaren Ertrag steht. Weshalb eben viele dieser Welten in einem Tempo ersonnen und erschrieben werden, das ihrer literarischen Überzeugungskraft und poetologischen Kohärenz nicht guttut.

9 In seinem Entwurf einer ,speläologischen Ästhetik des Computerspiels“ stellt Markus Rautzenberg fest: „Von ihren technischen Ermöglichungsbedingungen in der Softwareentwicklung bis hinauf zur Benutzerebene via Interface bestehen Computerspiele aus komplexen semiotischen Verweisungszusammenhängen, deren Logik auf der basalen, technischen Ebene einem kausalen ,Wenn/Dann'-Prinzip verpflichtet ist, welches in elektronische Systemzustände innerhalb der Prozessoren überführt wird. Auf der aisthetischen Ebene des Benutzers jedoch zeigt sich dieses Ineinander von Zeichen und Aktalisierung als ludischer Möglichkeitsraum [...], der nicht fixiert, sondern offen gestaltet und aktuell beeinflusst werden kann.“ Später führt er aus, dass die „Attraktivität von Computerspielen“ ganz wesentlich aus der „Exploration“ bestehe, wobei die „Orte“, die das Spiel bereitstellt, erst durch die „Interaktion des Spielers“ zu „Räumen transformiert werden und umgekehrt“. Markus Rautzenberg: Caves, Caverns and Dungeons. Für eine speläologische Ästhetik des Computerspiels. In: Benjamin Beil/Gundolf S. Freyermuth/Lisa Gotto (Hg.): New Game Plus. Perspektiven der Game Studies. Genres - Künste - Diskurse. Bielefeld 2015, S. 245-266, hier: S. 252 u. 254. 
Wie wir sehen, betrifft die Affinität zwischen Fantasy und Videospielen also vorwiegend eine in der Genrepoetik angelegte ästhetische Erfahrungsmodalität. Woraus folgt, dass sie sich auch auf Spiele erstreckt, die, was das Szenario betrifft, nicht unbedingt der Fantasy (ganz sicher nicht im Sinn der taxonomischen Genretheorie) zuzurechnen sind.

Um zu verstehen, was das alles bedeuten soll, mag es hilfreich sein, sich die medialen Differenzen in concreto vor Augen zu führen: Wenn mich ein Fantasy-Roman an einen wundersamen Ort führt, beispielsweise eine uralte, geheimnisvolle Ruine, muss ich mit dem Vorlieb nehmen, was mir die jeweilige Autorin, der jeweilige Autor zur Verfügung stellt. Die Beschreibung der Ruine mag für meinen Geschmack zu knapp ausfallen, weil ich die architektonischen Einzelheiten, das sinnliche Gepräge des Bauwerks gerne in größerer Detailliertheit in mich aufgesogen hätte; vielleicht ist sie mir auch zu umfänglich, insofern die gedruckten Worte meine eigene Vorstellungskraft in ihrer Entfaltung beeinträchtigen. Darüber hinaus muss ich, ganz buchstäblich, mitgehen, wenn die Figuren des Romans die Entscheidung treffen, die Ruine links liegen zu lassen, weil sie ihnen zu gefährlich erscheint oder weil sie noch am selben Tag die nächste Siedlung erreichen wollen. Hingegen stellt eine solche Ruine in einem Videospiel wie ELEX (Piranha Bytes, 2017) - das in der Gestaltung des Planeten Magalan die Zeitlichkeiten der Fantasy, der Science-Fiction und der Postapokalypse à la MAD MAX 2 (George Miller, AUS 1981) verbindet - ein Angebot an die Spielerinnen und Spieler dar, mit dem sie meistens verfahren dürfen, wie es ihnen beliebt: Es steht ihnen frei, das verfallene Gebäude aus der Ferne zu bewundern oder eine halbe Stunde damit zu verbringen, jeden seiner Winkel zu durchstöbern; je nach Laune können sie sich, obgleich sie vielleicht gerade auf dem Weg waren, etwas Dringliches zu erledigen, von der Ruine ablenken lassen; oder die Entscheidung treffen, dass sie sich die Erkundung für später aufheben; oder zu dem Schluss gelangen, dass sie, zumindest in dieser Spielwelt, mehr als genug Ruinen gesehen haben und jetzt viel lieber die Haupthandlung voranbringen wollen. ${ }^{10}$

10 In Hinblick auf die ELDER-SCROLLS-Spiele erläutert Britta Neitzel: „Neben diesen chronologischen und zielgerichteten temporalen Strategien finden sich jedoch in Spielangeboten auch nicht-gerichtete oder zirkuläre Zeitformen. Der Erfolg der ELDER SCROLLS-Reihe ist wahrscheinlich auch darauf zurückzuführen, dass sie den Spielenden Handlungsangebote macht, die unterschiedlichen Temporalitäten unterliegen. Einerseits können die Spielenden den Quests folgen - also auf ein Ende abzielen -, andererseits auch herumwandern und schauen, was sich so tut und was man noch so tun könnte in der virtuellen Welt. Man kann auch einfach die Aussicht genießen. Es ist quasi ein Urlaubsangebot, das es ermöglicht, ohne Zeitdruck zu handeln.“ Britta Neitzel: Involvement. In: Benjamin Beil/Thomas Hensel/Andreas Rauscher (Hg.): 
Um ein weiteres Beispiel zu geben: Wenn der Held in einem Fantasyfilm (oder einer Fantasy-Fernsehserie) entdeckt hat, dass er verraten worden ist, kann ich mir als Zuschauer wünschen, dass er Gnade vor Recht ergehen lässt und dem Schurken verzeiht (oder ihn umgekehrt einen Kopf kürzer macht); aus naheliegenden Gründen ist es mir aber nicht möglich, den Entschluss unseres Recken zu beeinflussen. Zumindest in jüngerer Zeit neigen Videospiele hingegen dazu, in vergleichbaren Fällen die Spielerinnen und Spieler entscheiden zu lassen: HORIZON ZERO DAWN (Guerrilla Games, 2017), das ebenfalls archaische und futuristische Zeitlichkeiten kombiniert, stellt uns beispielsweise vor die Wahl, ob wir Olin, der die Protagonistin Aloy - freilich, um seine entführte Familie zu schützen - an ihre Feinde verraten hat, töten oder verschonen wollen. Wie weitreichend solche Entscheidungen sind, steht auf einem anderen Blatt; in HORIZON ZERO DAWN jedenfalls hat Olins Wohlergehen keinen großen Einfluss auf den weiteren Handlungsverlauf. Das ändert aber nichts daran, dass die Spielerinnen und Spieler in solchen Momenten die ethische Haltung des Avatars bestimmen können; was zweifelsfrei einen Unterschied macht bezüglich der Frage, wie sie die Spielfigur wahrnehmen und wie es sich für sie anfühlt, mit dieser Figur durch die Welt zu streifen.

Oftmals sind die Entscheidungen der Spielerinnen und Spieler auch tatsächlich ein bestimmender Faktor, was, wenn man so sagen kann, die objektive Verfasstheit der Spielwelt betrifft. Die beiden zumeist dem Steampunk zugerechneten DISHONORED-Titel (Arkane Studios, 2012 und 2016) oder das im Gothic schwelgende VAMPYR (Dontnod Entertainment, 2018) sind drei von zahlreichen Spielen, bei denen die Vorgehensweise des Spielers - also die Frage, wie viele digitale Leichen er unnötigerweise produziert -, darüber entscheidet, wie hoffnungsvoll das Ende ist, das er erleben darf. Im Fall von VAMPYR betrifft dies immerhin das weitere Geschick der Hauptfigur Jonathan Reid, also die Frage, ob der Arzt auch als Vampir seine Menschlichkeit bewahrt und mit seiner Geliebten, der ebenfalls untoten Lady Ashbury, zusammen sein kann; oder ob er fortan als reißende Bestie durch die Schatten streifen wird. (Wobei sicherlich kein Mangel an Spielerinnen und Spielern herrscht, die es bevorzugen, sich Jonathan als einsamen, blutgierigen Supervampir vorzustellen.)

Hier sind nun einige Erläuterungen angebracht. Zunächst einmal will ich natürlich nicht darauf hinaus, dass die ästhetische Erfahrung, die das Videospiel bezogen auf die Fantasy realisiert, „besser“ oder „vollständiger“ wäre als jene, die andere audiovisuelle Medien oder die Literatur erlauben. Wohl aber

Game Studies. Wiesbaden 2018, S. 219-234, hier: S. 231. Vgl. zu den Zeitlichkeiten der ELDERSCROLLS-Reihe auch die SKYRIM-Analyse in diesem Kapitel. 
scheint sich die Vermutung zu bestätigen, dass es dem Videospiel aufgrund der Freiheiten, die es dem Spieler in Hinblick auf die Gestaltung seines Verhältnisses zur Spielwelt gewährt, eher möglich ist, die poetologische Zielsetzung des Genres - so wie sie sich mir darstellt - zu erfüllen.

Das betrifft wesentlich die Verheißungen der aus Geschichten gemachten Welt. Schließlich gehen die meisten kommerziellen Videospiele von der Situation aus, dass eine oder mehrere Spielfiguren in eine Welt versetzt werden und mit ihr interagieren müssen. Innerdiegetisch mag dem Avatar seine Umgebung vertraut oder fremd sein; aus Sicht der Spielerinnen und Spieler ist sie zunächst immer eine Terra incognita, die mehr oder weniger verwirrend, rätselhaft und herausfordernd sich darstellt. Es gibt dann einen Prozess, in dem die Einübung der Spielmechaniken, die Erschließung der Spielwelt und die Entfaltung der Narration sich verbinden. Fast zwangsläufig werden die Spielerinnen und Spieler im Verlauf dieses Prozesses erproben, wie umfänglich ihre Möglichkeiten sind, die Spielwelt zu beeinflussen; beziehungsweise ob es das Weltdesign gestattet, dass sie sich abseits eines vorgegebenen Pfades bewegen, dabei neue, unerwartete Räume eröffnen und erkunden, Geheimnisse finden und lüften und vielleicht auch etwas über die Hintergründe der Spielwelt erfahren. Die Gültigkeit dieses Prinzips besteht, so meine ich, nahezu unabhängig vom Szenario des jeweiligen Spiels, dem Spieltypus und dem Entwicklungsstand der Technik. ${ }^{11}$ Schon SUPER MARIO BROS. (Nintendo Research \& Development, 1985), bekanntlich nicht nur in Hinblick auf das Jump-'n'-Run-Genre eines der einflussreichsten Spiele überhaupt, lockte mit verborgenen Leveln; obzwar sich die berühmt-berüchtigte „Minus World“ oder „World -1“, die in der ersten Fassung des Spiels zu entdecken war, einem Programmfehler verdankte ... und man ihr zudem nicht zu entkommen vermochte. ${ }^{12}$

11 Marc Bonner schreibt: „Die Art und Struktur der heute meist dreidimensional ausgestalteten Welten hängt stark von den Spielmechaniken ab: Welche Handlungsoptionen erhält der Spieler? Welche Bewegungsmuster werden ihm ermöglicht? Die meisten Genres oder Spielmechaniken haben als Agens die Erkundung, Aneignung und Eroberung von Raum gemein.“ Und weiter heißt es: „Die Spielwelt muss in ihrer Gestaltung und Rhythmisierung auf multiple Wahrnehmungsmodi der Spieler eingehen - zwischen ludus und paidia, zwischen Stimmung und Chaos. Als raumzeitlich-manifestierte Spiegelung der Handlungenoptionen des Avatars sollen neben ludischen auch narrative Informationen kommuniziert werden.“ Marc Bonner: Welt. In: Beil/Hensel/Rauscher (Hg.): Game Studies, S. 129-151, hier: S. 129 u. S. 141.

12 Vgl. zu der Entwicklungsgeschichte ebenso wie zu den Mythen und Legenden, die sich um Nintendos Spielserie ranken: William Audureau: L'Histoire de Mario. 1981-1991 - L'ascension d'une icône. Houdan 2011.

Thomas Hensel erkennt in diesem Glitch-Level - beziehungsweise der Lage, in die er den Spieler bringt - eine geradezu existenziell aufgeladene „Ausweglosigkeit“. Vgl. Thomas Hen- 
Man könnte sich zu der Behauptung versteigen, dass dem Videospiel - so, wie wir es kennen - gar keine andere Wahl bleibt, als auf den ästhetischen Genuss einer aus Geschichten gebauten Welt zu setzen; und dass es darum immer Teil hat an der Genrepoetik der Fantasy. Bedenkt man den nun schon seit Jahrzehnten andauernden Welterfolg eines konsequent abstrakten Spiels wie TETRIS (Alexei Paschitnow, 1985), ist diese Behauptung sicherlich nicht haltbar. Betrachtet man das Gros der erfolgreichen Videospiele, ist sie aber auch nicht ganz falsch.

Vielleicht lässt sich die folgende These aufstellen: Spiele, die nach dem Prinzip der Open World gestaltet sind, stellen eine Apotheose der Fantasy in der zeitgenössischen Populärkultur dar, insofern sie grosso modo auf die freie Erkundung einer Welt gerichtet sind, die sich in räumlicher Weite und historischer Tiefe ausfaltet und dabei oftmals auch die ihrer Konstruktion zugrundeliegenden geschichtsphilosophischen oder metaphysischen Prinzipien enthüllt; was den Spielverlauf betrifft, so erweist es sich, dass die entsprechenden Welten auf der Mikroebene aus unzähligen kleinen Geschichten gemacht sind - die von NPCs erzählt und in Missionen oder Questen erlebt werden, sich dabei in der sinnlichen Erfahrung von zu entdeckenden und erkundenden Örtlichkeiten darbieten -, wohingegen das Spielerleben auf der Makroebene um eine große Geschichte strukturiert ist, die häufig das Schicksal der jeweiligen Welt betrifft, eine Entscheidungsschlacht zwischen guten und bösen Mächten, zumindest aber das Ende einer Epoche, den Umbruch einer historischen Zeitlichkeit. Und das alles gilt ebenso für Open-World-Titel wie die erwähnten ELEX und HORIZON ZERO DAWN, die sich an Zeitentwürfe der Science-Fiction anlehnen, und sogar für einen melancholischen Western wie RED DEAD REDEMPTION 2 (Rockstar Games, 2018). Aber auch Videospiele, die nur über enge und geschlossene Welten und mitunter nicht einmal über 3D-Grafik verfügen, zielen (insofern sie überhaupt narrativ organisiert sind) häufig auf den Genuss, der mit der Erfahrung und der Erkundung einer aus Geschichten gemachten Welt einhergeht und zwar selbst dann, wenn sie vorderhand nicht viel mit Fantasy zu tun haben mögen (im Sinn eines Sets an typischen Figuren, Handlungsfigurationen und Schauplätzen) und sich die Ergründung der Spielwelt vorwiegend in den Köpfen der Spielerinnen und Spieler vollzieht.

In diesem Sinn kann man sagen, dass das Videospiel als Medium wenigstens mit einem grundlegenden ästhetischen Konstruktionsprinzip der Fantasy sich

sel: „Know your Paradoxes!“ Das Computerspiel als multistabiles Bild. Mit einem Post Scriptum zur Genretheorie. In: ders./Britta Neitzel/Rolf F. Nohr (Hg.): „The Cake is a lie!“ Polyperspektivische Betrachtungen des Computerspiels am Beispiel von PORTAL. Münster 2015, S. 135-154, hier: S. 148. 
eng verbündet: eben der aus Geschichten gemachten, mythopoetischen Welt. Wie aber steht es um die anderen zwei Prinzipien, welche die Fantasy meines Erachtens prägen: Gut und Böse als Baustoff eines metaphysischen Weltentwurfs und natürlich die Eukatastrophe? Ich kann nicht erkennen, dass sich die privilegierte Beziehung zwischen Fantasy und Videospiel notwendigerweise auf diese beiden Prinzipien erstreckt. Wie wir sehen werden, sind Videospiele durchaus in der Lage, die Eukatastrophe mit aller Wucht und allem Zauber ins Werk zu setzen (auch im weiteren Sinne, als dynamischen Umschlag zwischen polaren affektiven Qualitäten) und ihre Welten in einer Weise zu entwerfen, dass von den Spielern eine entschiedene Bezugnahme auf die jeweiligen widerstreitenden Ideen von Gut und Böse verlangt wird.

Aus meiner Sicht verhält es sich aber nicht so, dass das Videospiel hier anderen Medien gegenüber im Vorteil wäre. Noch die Entscheidungen, die die Spielerinnen und Spieler treffen müssen, sind oft ebenso sehr auf die Gestaltung des eigenen Verhältnisses zu der Spielwelt gerichtet, und auf die Erfahrungspotenzialität derselben, wie auf die Positionierung des Avatars innerhalb eines - jeweils auszugestaltenden - Koordinatensystems von Gut und Böse. Vielleicht lässt sich sogar behaupten, dass die ethische oder metaphysische Dimension der Spielerentscheidungen bis zu einem gewissen Grad im Dienst der Welterfahrung steht, insofern etwa in HORIZON ZERO DAWN die Geheimnisse der geheimnisvollen und fluchhaften Vergangenheit (die infolge von menschlicher Hybris und Profitgier gestorbene und durch eine künstliche Intelligenz namens Gaia neu - und mitsamt mechanischen Dinosauriern - belebte Erde) nicht ganz dieselben sind, beziehungsweise nicht ganz dieselbe Bedeutung haben mögen, für eine Aloy, die Olin getötet hat, wie für eine, die eben dies nicht getan hat. Auch das trifft auf zahlreiche Spiele zu, die nicht, oder nur mit gewissen Abstrichen, der Fantasy zuzurechnen sind; Spiele also wie DISHONORED, VAMPYR oder eben HORIZON ZERO DAWN, die ich aus eben diesem Grund als Beispiele herangezogen habe.

Allerdings ist es sicherlich sinnvoll, die weltenbauende Kompetenz des Mediums an Spielen zu diskutieren, die in einem strengen, genrepoetischen Sinn der Fantasy zuzurechnen sind. $\mathrm{Zu}$ diesem Zweck habe ich zwei sehr unterschiedliche Titel ausgewählt: THE ELDER SCROLLS V: SKYRIM und THE BANNER SAGA. Während SKYRIM geradezu als Epitom des Fantasy-Videospiels gelten kann und das Prinzip der aus Geschichten gebauten Welt in schier unendlicher Vielgestaltigkeit verwirklicht, nutzt THE BANNER SAGA (was übrigens auch für seine zwei Fortsetzungen gilt) verschiedene Mechaniken und noch seine grafischen Gestaltungsmerkmale in sehr konsequenter Weise, um die Spielerinnen und Spieler in eine Welt zu versetzen, die zwar von mythopoetischem Zauber erfüllt ist, sich aber vor allem als einzige Folge ebenso folgenschwerer wie unabsehbarer Entscheidungen präsentiert. 
Ehe ich mich diesen beiden Spielen zuwende, scheint es mir jedoch nötig, einige Punkte theoretisch zu vertiefen. Das betrifft die Frage, wie sich das Verhältnis des Spielers zur Spielwelt konzipieren lässt; es betrifft außerdem die genretheoretischen Ansätze der Game Studies; schließlich betrifft es die Methodik zur Analyse von Videospielen. Man betritt hier gleich drei weite Felder, die schon einzeln zu großflächig sind, um ohne Weiteres vermessen zu werden. In diesem Sinn betrachte ich meine Überlegungen als vorläufig und bruchstückhaft; sie stellen Ansätze dar zu umfassenderen Erörterungen, die im Rahmen dieser Studie nicht zu leisten sind. Was freilich umgekehrt nicht heißen soll, dass die im Folgenden entwickelten Thesen an sich nur ein Notbehelf wären.

\section{Das ludische Oxymoron}

Wenn es um die Frage geht, welches Verhältnis die Spielerinnen und Spieler zu der Welt einnehmen, die ihnen ein Videospiel eröffnet, geht es zunächst um eine Idee von Bewegung. Die Welt darf und muss erschlossen werden; es ist ein prozesshaftes Ausloten der Grenzen und Möglichkeiten eines gegebenen Raums, das sich in physisch-sinnlicher Konkretion vollzieht. Das gilt unabhängig davon, ob wir es mit einem 3D-Open-World-Spiel oder einem Side-Scroller zu tun haben; es gilt auch unabhängig davon, ob man beispielsweise mit einer Magierin durch einen Wald läuft, oder ob man ein Ikon über eine stilisierte Karte steuert. Immer steht das Medium vor der Aufgabe, eine Welt erfahrbar zu machen, die in dem Maße lebendig wird, wie die Spielerinnen und Spieler sie sich aneignen.

Freilich sind es zunächst nicht diejenigen, die vor den Fernsehern und Monitoren sitzen, die sich bewegen, sondern die Spielfiguren. Andererseits, ganz stimmt das nicht. Denn die Spielerinnen und Spieler bewegen sich ja doch irgendwie, wenn sie ihre digitalen Abenteuer erleben. Zumindest rühren sie die Finger und Hände; und das müssen sie auch tun, wenn, im Wortsinn, etwas laufen soll in der Spielwelt. Dieser Bewegungsaufwand steht offenkundig in keinem Verhältnis zu den meist übermenschlichen, der Schwerkraft und sonstigen physikalischen Gesetzen spottenden Aktivitäten des Avatars. Aber es ist natürlich auch nicht so, dass die Spielfiguren ihre Großtaten völlig losgelöst von den Bemühungen der Spielerinnen und Spieler vollbringen würden. Vielmehr sind häufig Geschick und eine beachtliche Fingerfertigkeit vonnöten, um ein Spiel erfolgreich zu beenden; es gibt sogar Spiele - wie Hidetaka Miyazakis DARK-SOULS-Trilogie, der ich mich im vierten Kapitel dieser Studie widmen werde -, die als so schwer gelten, dass man vielerorts bereit ist, den Spielerinnen und Spielern, welche sie bemeistern, selbst einen gewissen Heroismus zuzubilligen. 
Zweifellos ist das eigenartige Ineinander von unauflöslicher Verbindung und radikaler Getrenntheit, was die Beziehung zwischen Spieler und Avatar betrifft - man könnte auch sagen: das Instrumentelle in der Bezugnahme von ersterem auf letzteren, die jedoch zugleich nach der ästhetischen Lebendigkeit des Instruments verlangt -, ein allgemeines Kennzeichen des Mediums Videospiel, und wurde auch oft als solches beschrieben. ${ }^{13}$ Allerdings stellt sich die Frage, ob in dieser grundlegenden Bestimmung etwas angelegt sein könnte, was in besonderem Maße auf Spiele zutrifft, die ihren Weltentwurf unter Bezugnahme auf die Genrepoetik der Fantasy realisieren. Immerhin haben wir es hier mit einer oxymoronischen Struktur zu tun - und wenn man Rosemary Jackson folgt, ist das Oxymoron, verstanden als ,figure of speech which holds together contradictions and sustains them in an impossible unity, without progressing towards synthesis“, die „basic trope“ der Fantasy. ${ }^{14}$

Nun verhält es sich so, dass Jacksons klassische Studie als beispielshaft gelten kann für das eingangs geschilderte Problem (also das definitorische Durcheinander, das sich unter anderem aus den Aporien der taxonomischen Genretheorie ergibt), ist es ihr doch wesentlich um die Fantastik zu tun, nicht um die Fantasy in einem erfahrungsästhetischen Sinn. Sie geht sogar so weit, die Werke der sogenannt hochliterarischen Fantastik, etwa von Kafka, Dostojewski

13 Thomas Hensel etwa spricht von der „ontologischen Uneindeutigkeit des Avatars“ und erläutert: „Wie man den Avatar im Einzelnen auch definieren mag - als Werkzeug, Sprite, Marionette oder Figur -, wesentlich zeichnet er sich durch eine paradoxale Doppelfigur aus - oder anders formuliert: durch eine Multistabilität -, nämlich einerseits als Protagonist einer Geschichte zu existieren und andererseits als Werkzeug des Spielers zu fungieren.“ Hensel: „Know your Paradoxes!“, S. 143. Bei Benjamin Beil und Andreas Rauscher heißt es: „Der Avatar wird nicht einfach zur Extension des Spielers; vielmehr stellt der Avatar einerseits die zentrale Kopplungsmöglichkeit zwischen Spieler und Spielwelt dar, andererseits akzentuiert er aber auch die Grenzen der virtuellen Welt. [...] Der Spieler wird nicht zum Avatar, sondern übernimmt lediglich Handlungsoptionen innerhalb der Spielwelt.“ Benjamin Beil/Andreas Rauscher: Avatar. In: Beil/Hensel/Rauscher (Hg.): Game Studies, S. 201-217, hier: S. 208. Und Carl Therrien schreibt: „Players control an avatar or a point of view as one would remote control an electric puppet or car, yet they get to incorporate these controls on a visceral level; they are looking at images that mimic to some extent our natural perception, but the typical screen is cluttered with arbitrary signs - such as the various assistance features highlighted earlier that represent a new form of visual narration, and complicate further the immersive posture.“ Carl Therrien: Immersion. In: Mark J. P. Wolf/Bernard Perron (Hg.): The Routledge Companion to Video Game Studies. New York/Abingdon 2014, S. 451-458, hier: S. 456.

Übrigens wäre zu untersuchen, ob und inwiefern die Entwicklung von Virtual-RealityTechnologien etwas Grundlegendes an diesem Verhältnis ändert.

14 Jackson: Fantasy, S. 21. 
oder Dickens - von ihr als fantasies bezeichnet - gegen jene der Fantasy - die sie als fairy tales adressiert - in Stellung zu bringen:

As narrative forms, fairy tales function differently from fantasies. They are neutral, impersonalized, set apart from the reader. The reader becomes a passive receiver of events, there is no demand that (s)he participates in their interpretation. Structurally, too, fairy tales discourage belief in the importance or effectiveness of action for their narratives are „closed“. Things „happen“, „are done to“ protagonists, told to the reader, from a position of omniscience and authority, making the reader unquestioningly passive. ${ }^{15}$

In dieser Perspektive erscheinen dann nicht nur Tolkien und C. S. Lewis, sondern selbst noch eine Autorin wie Ursula K. Le Guin als unpolitisch oder gar „sentimental and nostalgic“, da sie in ihren Romanen „problems of social order" unangetastet lasse. ${ }^{16}$ Auch Jackson unternimmt keinen Versuch, ihre großflächig gestreuten Anschuldigungen mittels einer genauen literaturwissenschaftlichen Analyse zu stützen, und ich meine bereits das Nötige zu dieser Art der Kritik gesagt zu haben - die sich hier übrigens auf Science-Fiction-Romane wie Le Guins The Left Hand of Darkness (1969) erstreckt. Hilfreicher scheint es mir, zu Jacksons Hauptthese zurückzukommen, der zufolge das Oxymoron als Sprachfigur „die Widersprüche verklammert und sie in unmöglicher Einheit aufrechterhält, ohne zur Synthese fortzuschreiten“. ${ }^{17}$ Bezieht man diesen Gedanken auf die Genrepoetik der Fantasy, die ich im vorigen Kapitel entwickelt habe, so ist festzustellen, dass mit den Kompositionsprinzipien der aus Geschichten gemachten „Sekundärwelt“ eine ganz eigene Ausdeutung des Oxymorons einhergeht. Schließlich ist es, wir erinnern uns, laut Tolkien von wesentlicher Bedeutung, die unmöglichen Welten der Fantasy in Erfüllung einer strengen Konstruktionslogik zu errichten, sodass noch eine grüne Sonne zugleich als Wunderlichkeit und notwendiger, nicht hinterfragbarer Bestandteil einer solchen „Sekundärschöpfung“ erscheinen mag. Das Oxymoron meint dann nicht mehr eine Sprachfigur, sondern eine ästhetische Erfahrungsmodalität, die unter Aufbietung der spezifischen Möglichkeiten des jeweiligen Mediums zu gestalten ist und auf eine Art Realismus des Irrealen zielt.

Vor diesem Hintergrund stellt sich die Frage, ob es eine Interpretation des Oxymorons gibt, die sich in besonderer Weise dazu eignet, die beschriebene Bewegungsidee des Videospiels zu erfassen, obendrein unter Bezugnahme auf die Genrepoetik der Fantasy. Mit Sicherheit lässt sich sagen, dass man Jacksons

15 Jackson: Fantasy, S. 154 [Herv. i. O.].

16 Jackson: Fantasy, S. 154-155.

17 Ich zitiere hier die Übersetzung von Michael Wedel, auf dessen Thesen ich sogleich zu sprechen kommen werde. Vgl. Wedel: Das audiovisuelle Oxymoron, S. 52. 
These sinnvoll auf audiovisuelle Medien übertragen kann. Michael Wedel hat dergleichen unternommen; auf der Grundlage von Katharine Fowkes' Überlegungen zum Fantasy-Film versucht er, das ,,audiovisuelle Oxymoron“ als ästhetisches Potenzial eines Blockbuster-Kinos $\mathrm{zu}$ definieren, das auf überaus avancierte Spezialeffekte und Soundtechnologien zurückgreifen kann, um die Zuschauerschaft in seinen Bann zu schlagen.

Auch Fowkes bestimmt Fantasy als „an umbrella category“, ${ }^{18}$ die, folgt man Wedel, Science-Fiction ebenso wie Horror und die Fantasy in der Tradition von Tolkien umfasst, meistens aber „eine Mixtur aus Elementen aller drei Genremodi“ aufweist. ${ }^{19}$ Im gegebenen Zusammenhang ist vor allem Fowkes' allgemeine Definition des Fantasy-Films von Bedeutung: „the audience must at the very least perceive an ,ontological rupture“ - a break between what the audience agrees is ,reality ' and the fantastic phenomena that define the narrative world.“ ${ }^{20}$ Und weiter: „The term ontological denotes the fact that fantastic phenomena are understood to really exist within the story-world - an existence as real as the reference world from which they break. ${ }^{\text {“21 }}$ Wedel übernimmt diese Definition, um sie im Folgenden auf „das Spiel des Genres mit Momenten der Wahrnehmungsillusion und in sich vielschichtigen Formen bildhafter Mehrdeutigkeit, seinen Rückgriff auf paradoxe Konstruktionen und oxymoronisch verfahrende Topoi des Selbst-Widerspruchs“ zu beziehen. ${ }^{22}$ Exemplarisch sind für ihn etwa jene Szenen aus HARRY POTTER AND THE DEATHLY HALLOWS: PART 1 (David Yates, GB/USA 2010), die auf die Gestaltung der Wirkungen von Zaubersprüchen abzielen. Nach Wedels Auffassung ist in solchen Szenen ein „synästhetisches Wahrnehmungsparadox“ realisiert, „das - zumal im Kontext der Fantasy - eine Form medialer ,Magie“ darstellt““. ${ }^{23}$ Es gehe hier um die „Hervorbringung von Situationen und Momenten, in denen Klänge die solide Form visueller Eindrücke annehmen und Bilder zu Figurationen sonorer Kraft und Fluidität mutieren“. ${ }^{24}$

Wedel führt aus:

Wenn solcherart, mit den Worten Jean-Luc Nancys, „Akustisches und Optisches einander gegenseitig affizieren“ und man den Eindruck hat, mit den Augen zu hören und mit den Ohren zu sehen, wenn visuelle Klänge und sonore Bilder sich einem reziproken Prozess

18 Katherine A. Fowkes: The Fantasy Film. Malden, Mass./Oxford/Chichester 2010, S. 2.

19 Wedel: Das audiovisuelle Oxymoron, S. 48.

20 Fowkes: The Fantasy Film, S. 5.

21 Fowkes: The Fantasy Film, S. 5 [Herv. i. O.].

22 Wedel: Das audiovisuelle Oxymoron, S. 52.

23 Wedel: Das audiovisuelle Oxymoron, S. 56.

24 Wedel: Das audiovisuelle Oxymoron, S. 55. 
der Metamorphose anheimgeben, ihre distinkten ontologischen Regime und sinnlichen Register zu oszillieren beginnen - in diesen Momenten erweist sich die Konstruktion der diegetischen Welt, in die wir als Zuschauer versetzt sind, schon von ihren wesentlichen filmischen Möglichkeitsbedingungen her als „different“ und andersartig. ${ }^{25}$

Die „mediale ,Magie““ des Fantasy-Films hat also etwas damit zu tun, dass den Zuschauern in der Filmerfahrung ein Wahrnehmen zuteil wird, das kategorial von der Alltagswahrnehmung differiert und insofern fantastisch ist, als es ein Sehen und Hören ermöglicht, in dem die Sinnlichkeit übersinnlich wird wobei die Erfahrungsfülle der „medialen ,Magie““ aufgrund der raumgestalterischen und technischen Erfordernisse ihrer Herstellung eigentlich allein im Kino gegeben ist. ${ }^{26}$ Es geht hier also um ein durch und durch medial organisiertes Selbsterleben, das im Vergleich zu einer Weltwahrnehmung, die von den entsprechenden Prozessen ästhetischer Erfahrung abgekoppelt ist, tatsächlich einen ontologischen Bruch inszeniert.

Vor dem Hintergrund dieser Überlegungen erscheint es nicht allzu weit hergeholt, die Existenz eines ludischen Oxymorons zu vermuten. Allgemein gesprochen korreliert es mit der grundlegenden Bewegungsidee des Mediums Videospiel, in der die Spielerinnen und Spieler einer von ihnen radikal getrennten, phantasmatischen Leiblichkeit - der des Avatars oder auch eines abstrakteren Agenten ihres spielenden Handelns, etwa einem Ikon, das eine Truppeneinheit symbolisiert - zugleich in ihrem konkreten, physisch-sinnlichen Erleben unauflöslich verbunden sind. Wenn es um die Fantasy geht, kommt noch ein Weiteres hinzu. Ich habe ja behauptet, dass der Genuss an diesem Genre sehr viel mit dem Eintritt in einen potenziell unendlich erweiterbaren Erzählraum zu tun hat, den die mythopoetische Welt eröffnet, indem sie eine Fülle an Geschichten bereitstellt; erschlossene und noch zu erschließende, ebenso wie halb verborgene oder nur erahnte Geschichten.

Indem das Videospiel den Spielerinnen und Spielern in der Erfahrung der Welt - als Prozess der räumlichen und zeitlichen Erschließung dieser Welt - eine Position zugleich inner- und außerhalb der Diegese zuweist, erlaubt es eine sehr

25 Wedel: Das audiovisuelle Oxymoron, S. 56.

26 Entsprechend geht Wedel bei seinen Darlegungen konsequent von der Rezeptionssituation im Kino aus. So schreibt er: „In der Beziehung zwischen dargestellter diegetischer Welt und Publikum impliziert das Ultrafeld des digitalen Surroundtons insofern eine radikale Veränderung, als es das Publikum nicht länger in Distanz zur auf der Leinwand sichtbaren Welt rückt, sondern immer dann danach strebt, den Zuschauer mitten in die Handlung zu versetzen, sobald das Potenzial des Surround-Sounds vollständig ausgeschöpft wird und die dazu nötigen ,Zugangspunkte“ bereitstellt.“ Wedel: Das audiovisuelle Oxymoron, S. 54. 
eigentümliche Form von Bezugnahme, die unter gewöhnlichen (sprich: nichtästhetischen) Umständen wohl in der Tat nur den Bewohnern der Faërie gewährt ist. Man ist mittendrin, bringt dieses Mittendrin erst hervor durch die eigenen Handlungen, und steht zugleich außerhalb; man erlebt die Gegenwärtigkeit, die damit sich verbindet, in jedem Moment teilzuhaben an der Auffaltung der Sekundärwelt, und ist, bezogen auf die Historizität der zu ergründenden Welt, zugleich jeder konkreten Zeitlichkeit enthoben.

Mit anderen Worten: Die Spielerinnen und Spieler, die im Medium Videospiel der Fantasy frönen, haben Anteil an dem gewissermaßen epischen Blick der Elben, die in einem Lidzucken den Aufstieg und Fall von Reichen bezeugen und denen die Jahrhunderte durch die Finger fallen wie Sandkörner - und sind zugleich dem Dasein einzelner, spielend sich verlebendigender Geschöpfe verbunden, die gar keine Wahl haben als Wurzeln zu schlagen in der Erde ihrer Geburt und ihres Sterbens und den flüchtigen, immer schon halb verronnenen Augenblick der Blüte (oder was wird aus einer Spielfigur, die niemand mehr spielt?), so gut zu nutzen, wie es ihnen eben möglich ist. Man könnte auch sagen, Thomas Hensel paraphrasierend, dass dem Avatar eine „Scharnierfunktion“ zukommt, nicht allein „zwischen Innen und Außen, zweidimensionaler Spielwelt und dreidimensionaler Spielerwelt“, sondern auch zwischen verschiedenen Zeitlichkeiten, wobei sich die Spielerinnen und Spieler, um es zugespitzt auszudrücken, spielend ihrer eigenen Zeitlichkeit entheben, indem sie sich in die Zeitlichkeit einer digitalen Figur versenken, der eben „eine fiktionale und eine faktuale Seite“ $z$ u eigen ist. ${ }^{27}$

Insofern hat es das Videospiel wirklich leichter mit der Fantasy. Nicht nur aus dem Grund, dass, anstelle des einzelnen Autors in der Schreibstube, mitunter Dutzende oder sogar Hunderte von Menschen über Jahre hinweg an der Ausgestaltung der Sekundärwelt arbeiten; sondern vor allem, weil sich das, was an dieser Sekundärwelt gut und böse sein mag, den Spielerinnen und Spielern aufgrund ihrer eigentümlichen Positionierung von Augenblick zu Augenblick im sinnlich-konkreten Welterleben erschließt; obgleich die Geschichten, die das jeweilige Spiel wirklich auserzählt, im Einzelfall an Trivialität kaum zu überbieten sein mögen.

\section{Muster, Formel, Modus}

Aber was erzählen die Spiele überhaupt? Und vor allem: Wie erzählen sie es? Kurzum, auf welche Weise(n) verwirklicht sich das Genre Fantasy im Medium

27 Hensel: „Know your Paradoxes!“, S. 143-144. 
Videospiel? Und noch allgemeiner: Was ist eigentlich der Status einzelner Genres, wenn es um Videospiele geht?

Eingangs sei an die grundlegende Einsicht von Rick Altman erinnert, der zufolge es keineswegs als gesichert gelten kann, dass der Begriff „Genre“ bezogen auf verschiedene Medien - etwa Film und Literatur - dasselbe bedeutet. ${ }^{28}$ Tatsächlich kann man, wenn es um das Videospiel geht, ohne weiteres ein mediales Spezifikum ausmachen, das auch hinsichtlich genretheoretischer Fragen von großer Bedeutung ist.

Andreas Rauscher schreibt:

Als wesentliche Grundlage für das Genreverständnis im Videospiel gilt die Kategorie des Gameplays. Sie wird als medienspezifische Besonderheit betrachtet, zu der bisher kein Äquivalent in anderen Medien existiert. Theorien, die sich um ein System für allgemeine Game Genre-Klassifikationen bemühen, konzentrieren sich gezielt auf die Bedeutung des Gameplays als Kernelement. ${ }^{29}$

Dabei versteht Rauscher - im Anschluss an Jesper Juul - unter „Gameplay“ „die Beeinflussung der Spielwelt durch den Spieler und die Weise, in der das Spiel tatsächlich gespielt wird“ und erläutert, dass Genrespiele auf dieser Grundlage „durch die Form der Interaktion der Spieler mit den Spielmechaniken und den zu erreichenden Spielzielen definiert“ werden. ${ }^{30}$

Diese Position, von Dominic Arsenault auf die Formel „gameplay comes first“" gebracht, ${ }^{31}$ ist in der Forschung zum Videospiel so weit verbreitet, dass sie beinah als common sense gelten kann; bereits seit vielen Jahren bildet sie einen unverrückbaren Fixpunkt in der genretheoretischen Diskussion zu diesem Medium. ${ }^{32}$ Ist die Sache damit erledigt? Keineswegs.

28 Rick Altman: Film/Genre [1999]. London 2006, S. 12.

29 Andreas Rauscher: Genre. In: Beil/Hensel/Rauscher (Hg.): Game Studies, S. 343-362, hier: S. 348.

30 Rauscher: Genre, S. 348; vgl. auch: Jesper Juul: Half-Real. Video Games between Real Rules and Fictional Worlds. Cambridge, Mass. 2005, S. 83.

31 Arsenault: Video Game Genre, Evolution and Innovation, S. 155.

32 So schrieb Mark J. P. Wolf bereits zu Beginn der Nullerjahre: „While the ideas of iconography and theme may be appropriate tools for analyzing Hollywood films as well as many video games, another area, interactivity, is an essential part of every game's structure and a more appropriate way of examining and defining video game genres.“ Mark J. P. Wolf: Genre and the Video Game. In: ders. (Hg.): The Medium of the Video Game. Austin 2001, S. 113-134, hier: S. 114. Und in einem Aufsatz von 2006 vertrat Thomas Apperley die These, das Hauptproblem „with conventional video games genres“ bestehe darin, dass sie weniger „a general description of the style of ergodic interaction that takes place within the game" seien, als vielmehr „loose aesthetic clusters based around video games’ aesthetic linkages to prior media forms“. 


\section{Arsenault etwa merkt an:}

The real question then does not concern the choice of criteria - as I demonstrated, everyone seems to think likewise - but rather [...] what comes after and around gameplay; for even once recognized sovereign, gameplay can still be broken down into a myriad components such as type of player skill involved, avatar abilities, progression structures, point of view, temporal unfolding, etc. ${ }^{33}$

Aus der Feststellung, dass dem Gameplay der Primat zukommt, wenn es um die Bestimmung der Genres des Videospiels geht, erwachsen also zahlreiche Schwierigkeiten.

„The problem of genre is not so much“, erläutert Arsenault,

that it is practiced with different criteria, but that these criteria are of different levels, and often a different nature. The difference between Arcade games and Console games has very little to do with the difference between Strategy and Action games, which is still very different from what separates Shooters from Fighting games, and we haven't gotten to First/Third-Person Shooters yet. And dare we point at Science-Fiction First-Person Shooters and their Historical counterparts, or to the Story-Driven and Emergent Historical FPS? The more we gaze, the deeper the abyss reveals itself. ${ }^{34}$

Wie kommen wir heraus aus diesem Abgrund? Zunächst ist es sicher hilfreich, mit Andreas Heidrich zu konzedieren, dass, wenn man bei Videospielen von Genres spricht, „verschiedene Weisen der Einteilung“ bezeichnet sein können. ${ }^{35}$ Für Benjamin Beil folgt aus dieser Erkenntnis eine zweite: jene nämlich, dass es sich bei Videospielen „um hochgradig hybride mediale Artefakte handelt“. ${ }^{36}$ In vielen Fällen ließe sich das Videospiel „nicht hinreichend über seine Spielelemente beschreiben“; vielmehr gebe es „eine Reihe anderer Kriterien - anderer medialer Ausdrucksformen -“, die ebenso berücksichtigt werden müssten, „etwa bestimmte narrativ-stilistische Elemente, die sich auch in anderen Genresystemen finden“. ${ }^{37}$ Ganz allgemein vertritt Beil die Auffassung, dass Genres

Thomas H. Apperley: Genre and Game Studies. Toward a Critical Approach to Video Game Cenres. In: Simulation \& Gaming 37 (2006), H. 1, S. 6-23, hier: S. 7.

33 Arsenault: Video Game Genre, Evolution and Innovation, S. 155-156.

34 Arsenault: Video Game Genre, Evolution and Innovation, S. 156.

35 Andreas Heidrich: Genre. In: Navigationen. Zeitschrift für Medien- und Kulturwissenschaft 11 (2011), H. 2, S. 85-87, hier: S. 85.

36 Benjamin Beil: Genrekonzepte des Computerspiels. In: GamesCoop: Theorien des Computerspiels zur Einführung. Hamburg 2012, S. 13-37, hier: S. 14.

37 Beil: Genrekonzepte des Computerspiels, S. 14. Aus diesem Grund ist Beil auch - zurecht, wie ich finde - der Ansicht, dass die Differenzen zwischen dem Videospiel und anderen (audiovisuellen) Medien stärker betont werden, als es nötig und sachgemäß ist; vor allem das Beharren auf der Interaktivität des Videospiels sei „ein häufig überstrapaziertes Argument beim intermedialen Vergleich“ mit dem Film. Beil: Genrekonzepte des Computerspiels, S. 15. 
medienübergreifend dazu dienen, „,bestimmte Erwartungshaltungen anzusprechen“ beziehungsweise als „Ausdruck von Erwartungshaltungen fungieren“ ${ }^{38}$ Auf dieser Grundlage diskutiert er dann verschiedene genretheoretische „Zuordnungskriterien“, als da wären „Spielmechanik“, „Raum/Perspektive“ sowie „Narration/Stil“, wobei er freilich einräumt, dass bei Genrebestimmungen immer gewisse „Unschärfen“ gegeben sind. ${ }^{39}$ Darüber hinaus erkennt Beil im Videospiel, wie gesagt, eine allgemeine Tendenz zu Hybridisierung, da sich die einzelnen Genres des Mediums je „aus einer Vielzahl unterschiedlicher Spielmechaniken, Darstellungsmodi und narrativ-stilistischer Elemente“ zusammensetzen ${ }^{40}$; zugleich ist er jedoch der Meinung, dass sich bestimmte Titel finden lassen, die, wie etwa SPORE (Maxis, 2008) oder BORDERLANDS (Gearbox Software, 2009), diese allgemeine Tendenz „durch die bewusste Verknüpfung etablierter Hauptgenres“ auf „eine Form der Neubildung" hin forcieren. ${ }^{41}$

Beils genretheoretische Thesen stammen aus dem Jahr 2012; betrachtet man nun die bereits zitierte Überblicksdarstellung von Andreas Rauscher, die 2018 veröffentlicht wurde, bekommt man den Eindruck, dass sich am Stand der Diskussion nicht sehr viel geändert hat. Auch Rauscher verweist auf die herausragende Bedeutung des Gameplays in Hinblick auf die Genretheorie des Videospiels, warnt aber unter Verweis auf „Hybridisierungsprozesse“ vor einer „puristischen Reduktion“422; und auch er diskutiert „Game-Genre-Typologien“433 und „Ästhetische Ansätze zur Genre-Analyse“ (wobei die Stichworte neben „Raum/Perspektive, Narration/Stil und Gameplay“ auch „Wiederholungen, Vergleiche und Diskursivierung“, den „Einsatz medienübergreifender Settings, die ästhetische und dramaturgische Erwartungen befördern, sowie die Anwendung dramaturgischer Standardsituationen“ umfassen). ${ }^{44}$ Darüber hinaus schlägt Rauscher vor, Rick Altmans pragmatisches genretheoretisches Modell auf das Videospiel zu übertragen, da die „Verknüpfung von Semantik und Syntax“ hier aufgrund „der zentralen Bedeutung des Gameplays“ noch wichtiger sei als im Film. ${ }^{45}$ In diesem Zusammenhang betont er, dass Altmans Modell

38 Beil: Genrekonzepte des Computerspiels, S. 20 u. S. 24.

39 Vgl. Beil: Genrekonzepte des Computerspiels, S. 20-28.

40 Beil: Genrekonzepte des Computerspiels, S. 31.

41 Beil: Genrekonzepte des Computerspiels, S. 31, vgl. auch S. 31-37.

42 Rauscher: Genre, S. 348.

43 Rauscher: Genre, S. 349, vgl. auch S. 349-350.

44 Rauscher: Genre, S. 350, vgl. auch S. 349-353.

45 Vgl. Rauscher: Genre, S. 353-356, hier: S. 353. Die zentrale These Altmans, auf die sich Rauscher beruft, lautet: „we can as a whole distinguish between generic definitions that depend on a list of common traits, attitudes, characters, shots, locations, set, and the like - thus stressing the semantic elements that make up the genre - and definitions that play up certain 
nicht von der „Annahme unveränderlicher Strukturen“ ausgehe, sondern postuliere, „dass jederzeit im Austausch zwischen Produzenten und Rezipienten neue Genres herausgebildet werden können“. 46

Ich will das Für und Wider der verschiedenen Ansätze, die zur Bestimmung von Genres im Videospiel herangezogen werden, nicht im Einzelnen diskutieren. Es scheint mir nicht geboten - und vielleicht auch nicht unbedingt hilfreich -, sich um eine systematisierende Perspektive zu bemühen, wenn das Verhältnis des Genres Fantasy zum Medium Videospiel in Rede steht. Sicher wäre eine kritische Überprüfung der innerhalb der Game Studies zirkulierenden genretheoretischen Postulate nötig, da viele von ihnen Gefahr laufen, in den Aporien der Taxonomie zu versacken. Für meine Fragestellung ist die Genretheorie der Game Studies aber nur insofern von Bedeutung, als sie ein Verständnis des Zusammenspiels von Fantasy-Poetik und Videospiel befördert.

Darum möchte ich nun kurz auf einige Überlegungen eingehen, die Thomas Hensel in Zusammenhang mit dem von ihm ausgemachten Genre der Escher Games angestellt hat. Aus Gründen, die ich noch darlegen werde, ist hier nämlich ein ähnliches Problem gegeben wie jenes, das sich in Hinblick auf die Beziehung zwischen Fantasy und Videospiel stellt. Das Tertium comparationis der von Hensel diskutierten Spiele bildet deren Verweis auf das Werk des Grafikers Maurits Cornelis Escher, der bekanntlich für seine Gestaltungen unmöglicher Gegenstände, Räume und Bildwelten berühmt geworden ist. Es geht hier um so unterschiedliche Spiele wie PORTAL (Valve, 2007), DANTE’S INFERNO (Visceral Games, 2010), GOD OF WAR III (SCE Santa Monica Studio, 2010), ALICE: MADNESS RETURNS (Spicy Horse, 2011) oder MONUMENT VALLEY (Ustwo, 2014) wobei es nicht von Belang ist, ob die Entwickler der jeweiligen Spiele die Reminiszenzen an Escher intendierten oder nicht.

Hensel grenzt sich zunächst von „essentialistischen Genretheorien“ ab, betont stattdessen - im Anschluss an ,poststrukturalistisch oder kognitionswissenschaftlich informierte Beiträge etwa zur filmwissenschaftlichen Genretheorie“ -, dass Genres ,,in stetem Wandel begriffen sind, ineinander übergehen, abrupt entstehen und ebenso abrupt wieder verschwinden können“; auch bezogen auf das Medium Videospiel gilt also, dass Genres „keine ahistorischen Entitäten“ darstellen, sondern vielmehr „Konzepte, die zwischen einzelnen Artefakten sowie ihren

constitutive relationships between undesignated and variable place-holders - relationships that might be called the genre's fundamental syntax." Altman: Film/Genre, S. 219.

46 Rauscher: Genre, S. 353. 
Produktions-, Distributions- und verschiedenen Rezeptionskontexten ausgemacht werden“. ${ }^{47}$ Auf der Grundlage dieser allgemeinen Feststellungen bezieht sich Hensel auf das literaturwissenschaftliche Modell von John G. Cawelti, „in dem Genres durch das Konzept der Formel ergänzt werden“"48; wobei Cawelti unter diesem Begriff „ein durch Iteration ausgebildetes Muster versteht“, also etwa „einen Figurentypus, ein narratives Motiv oder eine ästhetische Konvention“. 49 Für Hensel liegt die Bedeutung dieses Konzepts von Iteration darin, dass es greifbar macht, inwiefern sich „populäre Textstrategien nicht in einem einzelnen Text, sondern als Muster nur in mehreren Texten“ erkennen lassen. ${ }^{50}$

Er führt aus:

In diesem Horizont bezieht Cawelti Formel und Genre in einem Zwei-Phasen-Modell der (Literatur-)Analyse aufeinander: In der ersten Phase wird durch einen Vergleich möglichst vieler Texte eine Formel identifiziert; in einem zweiten Schritt wird die Formel benannt und bewertet, und erst diese Benennung und die Diskursivierung der Formel lassen sich gemäß Cawelti als ein Genre fassen. ${ }^{51}$

Im gegebenen Zusammenhang ist nun von Bedeutung, wie Hensel diese Konzeption nutzt, um die Escher Games als Genre zu fassen. Im Sinne Caweltis könne als deren Formel „das Iterations-Muster ,spielerische Herausforderung durch Puzzles, die auf multistabilen Bildern basieren“ ausgemacht werden“, wobei es sich um ein Muster handelt, das eine „Einordnung nach Spielmechanik sowie Ästhetik zum Ausdruck bringt“. ${ }^{52}$ Vor allem scheint mir interessant, dass die Formel, die Hensel beschreibt, für Spiele fruchtbar gemacht werden kann, die nach gängigen genretaxonomischen Zuteilungen in ganz verschiedenen Schubladen untergebracht werden müssten. Hensel nennt hier unter anderen GOD OF WAR III als Vertreter des Genres „Action-Adventure“, ECHOCHROME (SCE Japan Studio, 2008) als Vertreter des Genres „Puzzle Game“ und THE BRIDGE (The Quantum Astrophysicists Guild, 2013) als Vertreter des Genres „Independent Game“. Er erläutert: „Während ,Action Adventure“ eine Einordnung nach Narration ausdrückt, sagt ,Puzzle Game‘ eine Einordnung nach Spielmechanik und ,Independent Game‘ eine Einordnung nach Produktionssoziologie oder -ökonomie aus.“53

47 Hensel: „Know your Paradoxes!“, S. 145.

48 Hensel: „Know your Paradoxes!“, S. 145; vgl. John G. Cawelti: Adventure, Mystery, and Romance. Formula Stories as Art and Popular Culture. Chicago/London 1976.

49 Hensel: „Know your Paradoxes!“, S. 145.

50 Hensel: „Know your Paradoxes!“, S. 145-146.

51 Hensel: „Know your Paradoxes!“, S. 146.

52 Hensel: „Know your Paradoxes!“, S. 146 [Herv. i. O.].

53 Hensel: „Know your Paradoxes!“, S. 146 [Herv. i. O.]. 
Dem Escher Game kommt so gesehen ein zweifaches genretheoretisches Potenzial zu: Einerseits führe es „gängige essentialistische Genre-Theorien an die Grenzen ihrer Erklärungskraft“; wichtiger sei aber, dass das Escher Game

als eine Metaisierung des vorgestellten Genre-Konzepts verstanden werden darf, insofern es die Quintessenz dieses Genre-Konzepts ikonisch und ludisch pointiert - eine Quintessenz, die man ihrerseits fassen kann mit der Formel ,Multistabilität als Herausforderung ${ }^{54}$

Hensel erkennt im Escher Game also eine Möglichkeit, das Genre in selbstreflexiver Perspektive über die eigene „Kernlosigkeit“ oder - wahrscheinlich treffender - „Multistabilität“ nachdenken zu lassen. ${ }^{55}$

Für sich genommen ist das sicher ein spannendes Unterfangen; in einer allgemeineren Perspektive erlaubt es Hensels Ansatz aber auch, einer Lösung für das Problem, was das Genre Fantasy hinsichtlich des Mediums Videospiel bedeuten mag, zumindest näher zu kommen. ${ }^{56}$ Dann nämlich, wenn man Caweltis Iterations-Begriff, so wie Hensel ihn versteht, an das erfahrungsästhetische Genrekonzept rückbindet, das ich unter Bezugnahme auf die Theorien Hermann Kappelhoffs skizziert habe. Dann könnte man vielleicht sagen, dass sich „das Iterations-Muster ,spielerische Herausforderung durch Puzzles, die auf multistabilen Bildern basieren“" ebenso wohl als spezifischer Typus von ästhetischer Erfahrung fassen lässt, die mit den Escher Games verbunden ist, beziehungsweise von diesen hergestellt wird als eine Spielerfahrung, ein Spielgenuss. In dieser Perspektive findet die Faszination an einem Escher Game ihren Grund ,in Reflexionen sogenannter unmöglicher Figuren, also jener grafisch zweidimensionalen, scheinbar dreidimensionalen Konstrukte, die als Körper nicht vorkommen können“57, ist es doch ein wesentliches Merkmal von Eschers Kunst, dass sie „Oberfläche und Unterfläche [...] oder Außenfläche und Innenfläche als miteinander verschliffen, mithin als Doppelnatur ein und desselben Bildes vorführt und ins Bewusstsein hebt“. ${ }^{8}$ Der ästhetische Genuss, der hier in Rede steht, kann Spielen teilhaftig werden, die vorderhand sehr unterschiedlichen Genres angehören (etwa Action Adventure und Puzzle-Game), und es ist ihm ohne weiteres möglich, sich diverse Spielmechaniken, Szenarien, Handlungsschemata, Figurentypen und raum-zeitliche Konfigurationen anzueignen, sodass er also so-

54 Hensel: „Know your Paradoxes!“, S. 147.

55 Hensel: „Know your Paradoxes!“, S. 146.

56 Andreas Rauscher führt diesen Ansatz übrigens bei den „Ästhetischen Ansätzen zur GenreAnalyse“ unter den Stichworten „Wiederholungen, Vergleiche und Diskursivierung“. Vgl. Rauscher: Genre, S. 350.

57 Hensel: „Know your Paradoxes!“, S. 136.

58 Hensel: „Know your Paradoxes!“, S. 139. 
wohl in dem bombastischen und bluttriefenden Nihilismus von GOD OF WAR III als auch in der zauberisch-skurrilen Poesie eines MONUMENT VALLEY sich auszugestalten vermag - mal als eher randständiges Element der Spielerfahrung, mal als Zentrum und Gravitationspunkt der künstlerischen Konstruktion.

Wenn das zutrifft, lässt sich durchaus die Behauptung wagen, dass in den Escher Games ein ästhetischer Modus hervortritt, der sich definiert über die „,spielerische Herausforderung durch Puzzles, die auf multistabilen Bildern basieren“"; zumindest dann, wenn man sich Kappelhoff anschließt, der - im Rückgriff auf Christine Gledhills überaus einflussreiche Definition - Modi bestimmt als „spezifische Muster ästhetischer Organisation medialer Strukturen, in denen unterschiedliche Erfahrungsweisen von Raum und Zeit, von Körpern, Sprachspielen und sozialen Kräfteverhältnissen realisiert werden und eine gewisse Stabilität ausbilden“" ${ }^{59}$ Freilich käme man nicht umhin, wollte man die Escher Games als ästhetischen Modus genauer ergründen, die von Hensel angeführten Spiele einer sorgfältigen Analyse zu unterziehen, um zu begreifen, was der vermeintlich abstrakte Rätselgenuss beispielsweise mit Erfahrungsweisen von sozialen Kräfteverhältnissen zu tun haben könnte. Dafür ist hier nicht der Ort.

Wohl aber erhebt sich die Frage, was die Escher Games mit der Fantasy zu tun haben. Ich meine, dass hier ein analoger Fall vorliegt, wenn es darum geht, welche Stellung den jeweiligen ästhetischen Phänomenen (also den Escher Games und der Fantasy) zukommt in Hinblick auf die gängigen Videospiel-Genres und die Einordungsmerkmale, die zu ihrer Kategorisierung herangezogen werden. Nehmen wir als Beispiel WARHAMMER: END TIMES - VERMINTIDE (Fatshark, 2015); gut taxonomisch könnte man dieses Spiel wohl als Multiplayer Online First-Person Shooter bezeichnen, der über einen Nahkampf-Schwerpunkt verfügt und in einem Dark-Fantasy-Szenario angesiedelt ist. Die Spielerfahrung und der Spielgenuss, die sich mit einer Partie VERMINTIDE verbinden, unterscheiden sich also zweifellos erheblich von der Spielerfahrung und dem Spielgenuss, die ein Einzelspieler Retro-Rollenspiel wie PILLARS OF ETERNITY (Obsidian Entertainment, 2015) bereitet, das spielmechanisch unter anderem durch rundentaktische Kämpfe, ausführliche Dialoge mit Nicht-Spieler-Charakteren sowie die

59 Kappelhoff: Genre und Gemeinsinn, S. 98. Bei Gledhill selbst heißt es: „The notion of modality, like register in socio-linguistics, defines a specific mode of asthetic articulation adaptable across a range of genres, across decades, and across national cultures. It provides the genre system with a mechanism of ,double articulation', capable of generating specific and distinctively different generic formulae in particular historical conjunctures, while also providing a medium of interchange and overlap between genres.“ Gledhill: Rethinking Genre, S. 229. 
Erkundung einer isometrisch gestalteten und - was die Atmosphäre und die grundlegenden Bauprinzipien betrifft - gewissermaßen klassischen FantasyWelt geprägt ist, wobei die Steuerung der Spielfiguren über eine Point-andClick-Benutzeroberfläche erfolgt. Andererseits scheint mir die Vermutung nicht völlig abwegig, dass VERMINTIDE erfahrungsästhetisch ebenso viel mit PILLARS OF ETERNITY zu tun haben könnte wie mit einem Multiplayer Online First-Person Shooter in der Art von BATTLEFIELD v (EA DICE, 2018), das zwar demselben Genre zugerechnet wird, aber zur Zeit des Zweiten Weltkriegs spielt und seine Schauplätze, Waffen und Klassen entsprechend gestaltet - also zu den sogenannten Military-Shooters zählt.

Diese Vermutung ist wenigstens dann plausibel, wenn man die Fantasy als übergeordnete Kategorie begreift, die einem Modus im gledhillschen - beziehungsweise kappelhoffschen - Sinn entspricht. Auf dieser Grundlage könnte man wesentliche Annahmen der Genrepoetik, die ich im ersten Kapitel dargelegt habe, in eine Formel oder ein Iterations-Muster umgießen und zugleich mit den obigen Überlegungen zu der Beziehung zwischen Fantasy und Videospiel verbinden. Etwa so: Die spielerische Herausforderung der Fantasy zielt auf die Erschließung einer Welt, die sich in räumlicher Weite, historischer Tiefe und metaphysischer Regelhaftigkeit auffaltet, wobei Rhythmus, Zeitlichkeit und Moralität dieser Auffaltung durch die Spielerinnen und Spieler selbst bestimmt werden. Miteingeschlossen in eine solche Formel ist eine Idee der zentralen Spielerfahrung und des spezifischen Spielgenusses, die die Fantasy im Medium Videospiel bereithält. Man kann davon ausgehen, dass diese Formel - beziehungsweise die Spielerfahrung und der Spielgenuss, die sie beschreibt - sich unterschiedlichste Mechaniken, Narrationstypen und auch Szenarien anzuverwandeln vermag, weshalb die Tatsache, dass eine Spielwelt, wie es bei HORIZON ZERO DAWN der Fall ist, von Roboterdinosaurieren bevölkert wird, noch lange kein Ausschlusskriterium darstellt, um das entsprechende Spiel der Fantasy zuzuordnen.

In diesem Sinne also meine ich, dass Thomas Hensels Thesen zu den Escher Games ein Verständnis davon befördern, weshalb etwa VERMINTIDE und PILLARS OF ETERNITY, all ihren Differenzen zum Trotz, mit Fug und Recht als FantasySpiele bezeichnet werden können. Auch wenn das Argument theoretisch überzeugen mag - im Einzelfall müsste sich seine Stichhaltigkeit auf analytischer Ebene erweisen. Zum Abschluss dieses Kapitels will ich deshalb, wie bereits erwähnt, zwei Spiele auf ihre Weltentwürfe hin untersuchen (und auf das Verhältnis, welches die Spieler zu der jeweiligen Welt einnehmen), die vorderhand kaum mehr miteinander zu tun haben mögen als ein First Person Shooter und ein Retro-Rollenspiel. Um die Analyse von SKYRIM und THE BANNER SAGA sinnvoll perspektivieren zu können - und zugleich die nötige Transparenz bezüglich 
der Aussagekraft ihrer Ergebnisse herzustellen -, sollten jedoch noch einige methodische Fragen geklärt werden.

\section{Methodologische Vorüberlegungen}

Allgemein ist festzustellen, dass sich die Game Studies eher in Zurückhaltung üben, was die Analyse einzelner Spiele betrifft; namentlich gilt das für Untersuchungen, die nicht darauf abzielen, theoretische Überlegungen an diesem oder jenem Spiel zu exemplifizieren, sondern die Spiele selbst als Medium des Denkens begreifen. ${ }^{60}$ Zweifellos hat diese Zurückhaltung etwas damit zu tun, dass die Game Studies als recht junges Fach zunächst den Schwerpunkt darauf gelegt haben, die Möglichkeiten und Grenzen ihres Gegenstands in einer medientheoretischen Perspektive zu ergründen. Abgesehen davon weist das Videospiel eine gewisse Unverträglichkeit mit den Erfordernissen des universitären Arbeitsalltags auf, wie er sich in der heutigen Zeit darstellt. Gewiss ist es nicht unbedingt nötig, ein Spiel, das man zu analysieren gedenkt, in all seinen Facetten zu ergründen. Eine Einschränkung, die aber keineswegs zu der Annahme berechtigt, man könne ästhetisch hochstehende und komplexe Spiele verstehen, wenn man mal kurz in den Anfang hineingeschnuppert hat. Häufig genug gilt (oder gälte) denn eben doch, dass die Möglichkeit der sachgemäßen Analyse eines Videospiels nur dann gegeben ist, wenn die Wissenschaftlerinnen und Wissenschaftler, die diese Arbeit leisten möchten, zuvor eine eingehende Kenntnis des jeweiligen Spiels erworben haben, was die Funktionsweise der Mechaniken und das Kompositions-

60 In Bezug auf Videospiele, die dem Horrorgenre zuzurechnen sind, betont etwa Bernard Perron diesen Mangel. Vgl. Bernard Perron: Introduction. Gaming After Dark. In: ders. (Hg.): Horror Video Games. Essays on the Fusion of Fear and Play. Jefferson/London 2009, S. 3-13. Auch Martin Hennig erkennt ein Desiderat in der Auseinandersetzung mit einzelnen Videospielen; seines Erachtens geht es dabei jedoch um „vertiefende inhaltsanalytische Zugänge“. Martin Hennig: Spielräume als Weltentwürfe. Kultursemiotik des Videospiels. Marburg 2017, S. 32. Das von Benjamin Beil, Thomas Hensel und Andreas Rauscher herausgegebene Lehrbuch Game Studies verfügt über viele kleine Beispielanalysen, die jedoch aus meiner Sicht vorwiegend zur Illustration der in den jeweiligen Kapiteln behandelten theoretischen Ansätze dienen; das schmälert nicht ihren funktionalen Wert, markiert aber recht präzise die Grenze, die dem analytischen Engagement der Game Studies zumeist gesetzt ist. Vgl. Beil/Hensel/Rauscher (Hg.): Game Studies. Zu den wenigen Spielen, die in größerem Maßstab analytische Anstrengungen inspiriert haben, zählt übrigens SHADOW OF THE COLOSSUS, dem ich mich im folgenden Kapitel widmen werde; was daran liegen mag, dass dieses Spiel einerseits einen herausragenden Status als Kunstwerk einnimmt, andererseits recht überschaubar ist, was den Umfang betrifft. 
prinzip des Weltenbaus ebenso einschließt wie beispielsweise die Entwicklung des Schwierigkeitsgrades, die affektive Pointierung der Narration, die audiovisuellen Rhythmen, die in der interaktiven Gestaltung des Spielverlaufs sich entfalten, oder die Formen der Gemeinschaftsbildung in etwaigen Multiplayer-Spielmodi.

Es handelt sich um einen Prozess der Einsenkung in ein Spiel als ästhetischen Gegenstand, der - um ein Beispiel aus der vorliegenden Studie zu wählen - bei einem Titel wie DARK SOULS gut und gerne dutzende, wenn nicht hunderte Stunden in Anspruch nehmen kann. Um es noch einmal zu unterstreichen: Je nachdem, welche Zielsetzung die analytische Anstrengung verfolgt, was die Fragestellung und die Ausrichtung des Unterfangens ist, mag sich dieser Prozess durchaus abkürzen lassen. Das ändert aber nichts daran, dass der Aufwand, der bei der Analyse eines Videospiels betrieben werden muss, sehr hoch zu veranschlagen ist; mag sein unverhältnismäßig hoch, wenn man zum Vergleich danach fragt, wie viel Zeit die Lektüre eines (durchschnittlich langen und komplizierten) Romans oder die (gegebenenfalls auch wiederholte) Sichtung eines Films veranschlagt.

Vor allem dann wird dieser Aufwand ein Problem, wenn Unklarheit darüber herrscht, ob die Ergebnisse der Analyse ihn rechtfertigen können. Soweit ich sehe, hat bis heute niemand den Versuch unternommen, eine systematische Methodik zur Analyse von Videospielen zu entwickeln. ${ }^{61}$ Das ist vielleicht auch nicht weiter verwunderlich, insofern das Videospiel, bezüglich seiner Gestaltungsprinzipien und der Rezeptionssituation, die es herstellt, mal dem Film und mal der Literatur zu ähneln scheint, dann wieder mit keinem von beiden Medien eine nennenswerte Ähnlichkeit aufweist, dafür aber die Spielerinnen und Spieler mitunter in eine Position versetzt, die jener gleicht, die man bei der Betrachtung eines Gemäldes oder gar dem kontemplativen Hören eines Musikstücks einnimmt. $^{62}$

Die theoretischen Schwierigkeiten, die mit der Analyse von Videospielen, und der Konzeption einer entsprechenden Methodik, einhergehen, sind also beträchtlich. Es wäre recht merkwürdig, wenn ich den Anspruch erheben wollte, im Handstreich die Lösung zu präsentieren. Eine Methodik zur Analyse von

61 Clara Fernández-Vara hat eine Einführung in die Analyse von Videospielen verfasst, die aber meines Erachtens keine medienspezifische Methodik entwickelt, insofern sie dezidiert auf textuelle Analysen abhebt. Vgl. Clara Fernández-Vara: Introduction to Game Analysis. New York/London 2015.

62 Damit ereignet sich im Videospiel das, was Georg Bertram „Intermedialität“ nennt, im Sinne eines echten „Zusammenspiels der Sinne“. Georg W. Bertram: Kunst. Eine philosophische Einführung. Stuttgart 2016, S. 97. 
Videospielen bleibt ein wichtiges Desiderat der Forschung; ${ }^{63}$ meinerseits möchte ich mich auf den Versuch beschränken, eine bereits voll entwickelte und oft erprobte Methode aus dem Bereich der Filmwissenschaft in heuristischer Perspektive auf das Medium Videospiel zu übertragen. Das heißt: Ob diese Übertragung schlüssig und produktiv ist, wird sich - wenigstens im Rahmen dieser Studie - nicht auf der theoretischen Ebene entscheiden. Vielmehr sollte sich der Gewinn des Ansatzes an den Analysen selbst, ihren Ergebnissen, erweisen. Des Weiteren will ich betonen, dass sich meine methodischen Überlegungen zuvörderst auf das Genre beziehungsweise den Modus Fantasy beziehen; daraus folgt nicht, dass sie jegliche Gültigkeit einbüßen würden, wenn sie beispielsweise auf ein Escher Game wie PORTAL oder einen Military-Shooter wie BATTLEFIELD V gerichtet wären. Wohl aber, dass sich meine Argumentation an der im ersten Kapitel dargelegten Genrepoetik und den auf den vorangegangenen Seiten entwickelten Thesen zum Verhältnis von Fantasy und Videospiel orientiert.

Was aber heißt das? Wenn die Annahme zutrifft, die besondere Kompetenz des Mediums Videospiel in Hinblick auf die Fantasy hänge damit zusammen, dass die Spielerinnen und Spieler in der Erkundung der jeweiligen fantastischen Welt sowohl die audiovisuelle Rhythmik als auch die spezifische Zeitlichkeit und mitunter sogar die Moralität der Entfaltung dieser Welt bestimmen wenn dies also zutrifft, braucht es eine Methode, welche in der Lage ist, die raum-zeitlichen Konstruktionsprinzipien audiovisueller Bilder aufzuschließen. Wohlgemerkt: nicht, um sie ins Verhältnis zu einer vorgängigen oder gar vormedialen Wirklichkeit zu setzen, sondern um die künstlerische Eigengesetzlichkeit der jeweiligen Weltentwürfe zu ergründen. ${ }^{64}$ Sollte etwas an der zentralen These diese Studie dran sein, der zufolge die Fantasy darauf zielt, eine Sehnsucht nach dem ganz Anderen als ästhetisches Gefühl zu gestalten, muss die Methode zudem dazu taugen, die Herstellung einer Spielerfahrung, eines Spielgenusses beschreibbar, greifbar, nachvollziehbar zu machen. Schließlich sollte

63 Andreas Rauscher und Willem Strank haben einen Band angekündigt, der über Methoden der Videospiel-Analyse unterrichten soll. Bedauerlicherweise war es mir nicht möglich, diesen Band vor Drucklegung der vorliegenden Studie zu rezipieren; es wird sich zeigen, inwieweit er dazu angetan ist, den geschilderten Missstand zu beheben. Vgl. Andreas Rauscher/Willem Strank (Hg): Close Playing. Methoden der Videospiel-Analyse. Wiesbaden 2020.

64 Man müsste meinen, dass kein Mensch auf den Gedanken käme, die Welten von Videospielen repräsentativ zu „lesen“. Doch dem ist nicht so. Wie ich im fünften Kapitel dieser Studie zu zeigen versuche, besteht ein Problem der Diskussion um die Gewaltinszenierungen des Mediums darin, dass die ideologiekritischen Ansätze dazu neigen, die „Inhalte“ von Videospielen - also das, was sie „darstellen“ - unmittelbar als Aussagen über die alltägliche Wirklichkeit zu interpretieren. 
die Methode auch über eine Idee davon verfügen, worin die politische Relevanz der Kunst besteht; oder anders gesagt: was die genuin ästhetische Dimension einer Politik der Kunst sein mag. Dies ist unabdingbar, weil die Kritik an der Fantasy letztlich als politische Kritik sich darstellt. Wir haben bereits mehrfach gesehen, dass die Art und Weise, wie dieses Genre seine Welten, Figuren und Geschichten konstruiert, grundsätzlich im Verdacht steht, reaktionäre, totalitäre oder gar offen faschistische Neigungen zu befördern. Das Kategorische des Vorwurfs verlangt nach einer entsprechenden Antwort - und diese ist vor allem in einer methodischen Darlegung zu suchen, da es hier wesentlich um die Frage geht, inwiefern Kunst ein politisches Machen ist, und worin dieses Machen bestehen könnte.

Meines Erachtens erfüllt die von Hermann Kappelhoff entwickelte filmanalytische Methodik alle hier genannten Erfordernisse. Sie ist als Entsprechung zu der, ebenfalls aus Kappelhoffs Schriften hergeleiteten, erfahrungsästhetischen Genretheorie zu verstehen, die ich im ersten Kapitel skizziert habe. Das heißt, sie stellt einen Versuch dar - unter Bezugnahme vor allem auf die deleuzianische Filmphilosophie, die Neophänomenologie in der Tradition von Vivian Sobchack und Helmuth Plessners Konzept der „Ausdrucksbewegung“ - die audiovisuellen Bilder selbst als Modus des Denkens zu erfassen.

Ich will nun knapp erläutern, was aus meiner Sicht das Entscheidende an dieser Methodik ist, und inwiefern ihre Grundannahmen für die Arbeit mit dem Medium Videospiel von Relevanz sein könnten. Auch hier ist ein caveat angebracht. Kappelhoff hat seine Methode zur Analyse audiovisueller Bilder über viele Jahre hinweg entwickelt, dabei auch stetig weiterentwickelt - etwa als eMAEX-System (electronically based media analysis of expressional movement images) ${ }^{65}$ - und in zahlreichen Aufsätzen und mehreren Monografien diskutiert. Meine Darstellung einiger Grundzüge dieser Methodik geht also notwendig mit Simplifizierungen einher. Was dieses Vorgehen rechtfertigt, so hoffe ich, ist seine pragmatische Nutzbarkeit. Es gibt nun mal, wie gesagt,

65 Selbiges „kombiniert ein systematisiertes filmanalytisches Vorgehen mit einer webbasierten Infrastruktur, die eine multimodale Aufbereitung analytischer Studien ermöglicht“. Kappelhoff: Genre und Gemeinsinn, S. 124. Vgl. auch: Hermann Kappelhoff/Jan-Hendrik Bakels/ Hye-Jeung Chung/David Gaertner/Sarah Greifenstein/Matthias Grotkopp/Michael Lück/Christian Pischel/Cilli Pogodda/Franziska Seewald/Christina Schmitt/Anna Steininger: eMAEX. Ansätze und Potentiale einer systematisierten Methode zur Untersuchung filmischer Ausdrucksqualitäten. In: http://www.empirische-medienaesthetik.fu-berlin.de/media/emaex_me thode_deutsch/eMAEX-_-Ansaetze-und-Potentiale-einer-systematisierten-Methode-zur-Unter suchung-filmischer-Ausdrucksqualitaeten.pdf?1401464494 [letzter Zugriff: 12.05.2020]. 
keine systematische Methodik zur Analyse von Videospielen, auf die man umstandslos zurückgreifen könnte. Wenn man das ästhetische Potenzial einzelner Spiele ergründen will, hat man also nur die Wahl, entweder (mehr oder weniger ausgewiesene) Methoden, die an anderen Gegenständen entwickelt wurden, auf dieses Medium zu übertragen oder gewissermaßen im Blindflug eine Methode als work in progress $\mathrm{zu}$ entwickeln, während man die analytische Arbeit unternimmt. Das erstgenannte Vorgehen ist sicherlich sinnvoller, da es den eigenen Ansatz transparent und mithin kritikfähig macht. Zumindest dann, wenn deutlich wird, auf welche Methode man sich bezieht und was man von ihr zu übernehmen sucht.

Was also möchte ich von Kappelhoffs Theorie übernehmen? ${ }^{66}$ Beginnen wir mit dem Grundsätzlichen. Kappelhoff schlägt „einen methodischen Ansatz der Analyse audiovisueller Bilder“ vor, „der sich auf audiovisuelle Bilder als $\mathrm{Me}$ dien der Wahrnehmung bezieht“. ${ }^{67}$ Das heißt: „Medien, durch die zuallererst hergestellt wird, was wir allzu beiläufig als gegeben voraussetzen: die phänomenale Wirklichkeit, die wir als eine gemeinschaftlich geteilte Wirklichkeit erleben."68 In dieser Perspektive sind audiovisuelle Bilder nicht als „gegebene Repräsentationen“, sondern „als technische Modellierung historisch generierter Wahrnehmungsformen" zu begreifen. ${ }^{69}$ Aus diesen Annahmen ergeben sich einige weitreichende Schlussfolgerungen.

Kappelhoff erläutert:

Wir verstehen Filmgeschichte in diesem Sinne als eine Geschichte der Poetologien audiovisueller Bewegtbilder, die sich als Blaupausen für die Analysen verschiedenster medialer und institutioneller Ausdifferenzierungen audiovisueller Bewegtbilder nutzen lassen. Wir adressieren darin die Grundmuster eines Denkens in Bildräumen und Wahrnehmungsszenarien, das erst in der Aneignung audiovisueller Bewegtbilder im Prozess filmischer Rezeption hervorgebracht wird. D. h., wir begreifen die filmische Rezeption keineswegs als eine reproduzierende Aktivität, die nur wiederholend entschlüsselt, was im Film als (narratives) Sinngebilde mehr oder weniger strikt vorgegeben ist. Vielmehr gehen wir von einer Interaktion zwischen Rezipient und audiovisuellem Bewegtbild aus, einem konkreten Machen im

$6 \mathbf{6}$ Ich habe mich vor längerer Zeit an einer zusammenfassenden Darstellung der kappelhoffschen Methode versucht, die ich damals als „Bildraum-Theorie“ bezeichnet habe; obgleich diese Darstellung - soweit ich sehe - im Wesentlichen noch immer gültig ist, schien es mir geraten, unter Bezugnahme auf die aktuellen Schriften Kappelhoffs einen neuen Anlauf zu unternehmen. Vgl. Daniel Illger: Heim-Suchungen. Stadt und Geschichtlichkeit im italienischen Nachkriegskino. Berlin 2009, S. 31-43.

67 Hermann Kappelhoff: Kognition und Reflexion. Zur Theorie filmischen Denkens. Berlin/ Boston 2018, S. 6 [Herv. i. O.].

68 Kappelhoff: Kognition und Reflexion, S. 6.

69 Kappelhoff: Kognition und Reflexion, S. 9-10. 
Medienkonsum, das den sinnhaften Zusammenhang eines filmischen Bildes erst herstellt. $^{70}$

Und weiter heißt es:

Damit wird die Rezeption selbst als eine Form der Produktion, die rezeptive Aneignung audiovisueller Bewegtbilder als ein genuiner Akt des Herstellens avisiert. Wir sprechen deshalb von der Rezeption audiovisueller Bilder als einer Poiesis des Filme-Sehens. ${ }^{71}$

In der Perspektive der kappelhoffschen Methodik ist mit der „Poiesis des FilmeSehens“ eine Aktivität der Zuschauerinnen und Zuschauer bezeichnet, die erst herstellt, was man gemeinhin unter einem „Film“ versteht. Die Poiesis des FilmeSehens nämlich „produziert Bild-Räume, in denen perzeptive Schemata, affektive Dynamiken, kognitive Konzepte und semiotische Prozesse in veränderlichen Konfigurationen aufeinander bezogen werden". ${ }^{72}$ Von wesentlicher Bedeutung ist nun zweierlei. Einmal die Feststellung, dass sich die Poiesis des Filme-Sehens weder „auf die Reproduktion der in audiovisuellen Bildern vorgegebenen perzeptiven oder kognitiven Schemata der Alltagswahrnehmung“ noch auch ,auf einen bloßen Nachvollzug der semiotischen oder narrativen Logiken kulturindustrieller, journalistischer, künstlerischer oder genrepoetischer Konzepte und Inszenierungsweisen“ reduzieren lasse. ${ }^{73}$ Zum anderen die These, dass in der Poiesis des Filme-Sehens „zwei Wahrnehmungsakte [...] konstitutiv miteinander verzahnt sind“. 74

\section{Nämlich:}

ein Sehen und Hören, das die technische Bildproduktion bestimmt und von dieser modelliert oder perpetuiert wird, sowie ein Sehen und Hören, das von Zuschauern als eigenes körperliches Erleben hervorgebracht werden muss, um die Sinnesdaten des audiovisuellen Materials in filmische Bilder zu transformieren. ${ }^{75}$

Kappelhoff führt aus, dass beide Wahrnehmungsakte stets ,in ein komplexes Gefüge sozio-ökonomischer, kultureller und politischer Bedingungen von Techniken, Konventionen, Vorbildern und kognitiven Operationen“, mithin „in eine gemeinschaftlich geteilte Weltsicht eingebettet und auf diese bezogen seien“,

70 Kappelhoff: Kognition und Reflexion, S. 10. Das „Wir“, das Kappelhoff hier anführt, bezieht sich auf die Kolleg-Forschungsgruppe „Cinepoetics - Poetologien audiovisueller Bilder“, die er zusammen mit Michael Wedel leitet.

71 Kappelhoff: Kognition und Reflexion, S. 10 [Herv. i. O.].

72 Kappelhoff: Kognition und Reflexion, S. 14 [Herv. i. O.].

73 Kappelhoff: Kognition und Reflexion, S. 14.

74 Kappelhoff: Kognition und Reflexion, S. 14.

75 Kappelhoff: Kognition und Reflexion, S. 14. 
und zwar gleichviel, ob man die audiovisuellen Bilder „als dokumentarisch, fiktional oder künstlerisch experimentell“ klassifizieren möchte. ${ }^{76}$

Auf dieser Grundlage kann er seine zentrale These formulieren:

Nicht die Filmproduktion per se, sondern der Kulturkonsum audiovisueller Bilder, die Poiesis des Filme-Sehens bringt den Diskurs filmischer Bilder als ein sozial, kulturell und historisch situiertes ,Denken der filmischen Bilder hervor. $^{77}$

Anders gesagt: „Der Diskurs filmischer Bilder ist als Produkt der Poiesis des Filme-Sehens zu analysieren und zu rekonstruieren.“78 Oder noch pointierter: „Das Werk, in dem die Poiesis des Filme-Sehens ihr Ziel hat, ist das Denken filmischer Bilder.“79

Wie nun können diese Überlegungen auf das Medium Videospiel übertragen werden? Zunächst das vermeintlich Selbstverständliche: Selbst wenn die Entwicklung der Hardware dafür sorgt, dass die grafischen Darstellungen aufwendig produzierter Videospiele mitunter eine fotorealistische Qualität annehmen - die Welten des Videospiel bleiben durch und durch künstliche und künstlerische Räume, die aus der Logik ihrer ästhetischen Konstruktionsprinzipien heraus begriffen werden müssen. Dann, und nur dann, lässt sich erfassen, inwieweit auch die technische Bildproduktion dieses Mediums ein Sehen und Hören modelliert und perpetuiert, das in dieser Modellierung und Perpetuierung eine Form der gleichermaßen affektiven wie reflexiven Selbst- und Weltwahrnehmung eröffnet, die so nur in der spezifischen ästhetischen Erfahrung, die mit dem jeweiligen Spiel einhergeht, zu haben ist. Das heißt: Videospiele erzeugen audiovisuelle Konfigurationen und Rhythmiken, die eine „gemeinschaftlich geteilte Weltsicht“ hervorbringen; zugleich sind es diese audiovisuellen Konfigurationen und Rhythmiken, die den Spielerinnen und Spielern erlauben, sich in der Spielerfahrung auf die solcherart gestaltete Weltsicht zu beziehen.

So gesehen sind Videospiele, nicht anders als Filme, „Medien der Wahrnehmung“, welche „die phänomenale Wirklichkeit, die wir als eine gemeinschaftlich geteilte Wirklichkeit erleben“ und gerne als gegeben voraussetzen, zuallererst herstellen. Und so gesehen kann man auch von einer „Poiesis des Videospiele-Spielens“ sprechen. Was das bedeutet, mag in der Tat deutlicher werden, wenn wir uns an die Thesen zum Verhältnis von Videospiel und Fantasy erinnern, die ich auf den vorangegangenen Seiten zu entwickeln versucht

76 Kappelhoff: Kognition und Reflexion, S. 14.

77 Kappelhoff: Kognition und Reflexion, S. 14.

78 Kappelhoff: Kognition und Reflexion, S. 14.

79 Kappelhoff: Kognition und Reflexion, S. 14. 
habe. Vor dem Hintergrund der Überlegungen Kappelhoffs scheint es sinnvoll, dieses Verhältnis noch einmal anders zu perspektivieren. Vielleicht lässt sich sagen, dass die Welt des Videospiels, was ihre grundlegenden Koordinaten und allgemeinen Konstruktionsprinzipien betrifft, etwas Gesetztes ist, prima facie eine stabile Referenz der Spielerfahrung. Aber zugleich gestalten die Spielerinnen und Spieler diese Welt; mehr noch: In gewisser Weise bringen sie die Spielwelt erst hervor, indem sie ihr, vermittelt über die Handlungen des Avatars, eine Zeitlichkeit und mitunter eine Moralität verleihen. So können sie die audiovisuelle Rhythmik des Spiels in der Rhythmik ihres Spielens einer je spezifischen Modulation zuführen - und in diesem Sinne auch die eigene Spielerfahrung, den eigenen Spielgenuss bestimmen.

Das wäre eine Möglichkeit, sicher nicht die einzige, das Konzept einer Poiesis des Videospiele-Spielens annäherungsweise zu erfassen. Es mag sein, dass es etwas Wesentliches am Medium Videospiel, und der Praxis des Umgangs mit ihm, überhaupt beschreibt; jedoch realisieren sich in ihm gleichermaßen die Grundzüge einer Genrepoetik der Fantasy.

So weit, so gut. Doch wie steht es um die Politik? Wenn man die basalen Annahmen der skizzierten Methode ernstnimmt, hat das unmittelbar Konsequenzen bezüglich der Frage, was das Politische an der Kunst ausmacht.

Kappelhoff schreibt:

Die Bildräume audiovisueller Bewegtbilder werden von den Rezipienten körperlich erschlossen, sie werden verkörpert als seien es Wahrnehmungsfigurationen der Alltagsrealität - ohne mit der Alltagswahrnehmung identisch zu sein. [...] Die medial generierten Wahrnehmungsprozesse des audiovisuellen Bewegtbildes sind zuerst ein reales, physisch-sinnliches Erleben und werden dann in imaginäre Vorstellungen transformiert, denen man Bedeutung zuschreiben und Sinn abgewinnen kann. ${ }^{80}$

Wie bereits ausgeführt, fasst die Poiesis des Filme-Sehens „die Interaktion der Rezipienten mit den audiovisuellen Bildern“ also als „Herstellungsprozess“; und die „repräsentationale Relation, der Wirklichkeitsbezug, ist ein genuin in diesem Prozess hergestelltes Produkt. Er bezieht sich stets auf eine fingierte Wirklichkeit. “81 Das heißt, in der Poiesis des Filme-Sehens - und dasselbe gilt wohl für die Poiesis des Videospiele-Spielens - vollzieht sich ein Denken

welches die Wahrnehmungsverhältnisse, d.i. die historisch-politischen, medialen und ästhetischen Bedingungen der Erfahrungswirklichkeit selbst reflektiert und manipuliert. Somit bringt die Poiesis des Filme-Sehens eine filmische Wahrnehmungswelt hervor, die

80 Kappelhoff: Kognition und Reflexion, S. 54.

81 Kappelhoff: Kognition und Reflexion, S. 68. 
sich als eine fingierte Wirklichkeit auf die alltägliche Erfahrungswirklichkeit bezieht, indem sie zu der gemeinschaftlich geteilten Alltagswelt in ein Verhältnis von Differenz und Vergleichbarkeit tritt. ${ }^{82}$

In dieser Perspektive erhält die Frage nach der politischen Dimension audiovisueller Bilder eine spezifische Bedeutung. Sie meint dann nicht mehr den Versuch, die kinematografischen Bilder daraufhin zu untersuchen, inwieweit ihre Konstruktionen mit dem übereinstimmen, was die Straße, das Haus, der Baum, der Mann und die Frau in unserer Alltagswahrnehmung und für unser Alltagsbewusstsein bedeuten, oder, anders ausgedrückt, ob und inwiefern sie einer der ästhetischen Erfahrung vorgängigen Idee oder Fantasie von Wirklichkeit oder eben dieser oder jener politischen Haltung gegenüber der Wirklichkeit entsprechen. Vielmehr zielt sie nun darauf, in der Analyse der Abweichung des Bildes der Straße, des Hauses, des Baumes, des Mannes und der Frau von dem vermeintlich Bekannten das aufzudecken, worin die audiovisuellen Bilder etwas zur Anschauung bringen, was sonst nicht gesehen werden kann, und dem Denken damit neue Positionen, neue Räume und also Potenziale eröffnet:

Erst die ästhetische Transformation der äußeren Wirklichkeit hebt an den alltäglichen Erscheinungsformen eine spezifische Phantasmatik, eine unvermutete Bildlichkeit hervor. Sie lässt ein Bild entstehen, das nicht auf etwas zurückweist, was ohnehin bereits da ist. Vielmehr rückt dieses Bild die sinnlich-physische Realität ins Sichtbare, die den historischen Grund unserer Subjektivität ausmacht, die gesellschaftliche Form, als welche unsere subjektive Wahrnehmungs- und Empfindungsweise überhaupt erst erkennbar ist. ${ }^{83}$

Wenn Kappelhoff recht hat, geht es also nicht an, die politische Analyse audiovisueller Bilder als Interpretation gegebener Sachverhalte oder repräsentierter Inhalte zu vollziehen. In Hinblick auf das Videospiel mag diese Einsicht besonders wichtig sein, da man in sehr vielen Spielen (und nahezu allen FantasySpielen) nicht zuletzt damit beschäftigt ist, mit mehr oder weniger real gestalteten Waffen eine Unmenge an mehr oder weniger real gestalteten Feinden ums digitale Leben zu bringen. Etwas formelhaft könnte man sagen, dass sich die politische Dimension audiovisueller Analyse vielmehr dann erschließt, wenn man die jeweiligen Welten in ihrer Abweichung von der Alltagswirklichkeit der raum-zeitlichen Eigengesetzlichkeit ihrer Konstruktion - zu bestimmen sucht und das so erfasste ästhetische Potenzial in einem nächsten Schritt auf soziale Konflikte, die Möglichkeiten und Grenzen spezifischer Gemeinschaftsentwürfe oder die Historizität der eigenen Gegenwart bezieht.

82 Kappelhoff: Kognition und Reflexion, S. 70.

83 Hermann Kappelhoff: Realismus. Das Kino und die Politik des Ästhetischen. Berlin 2008, S. 63. 
Aus Kappelhoffs Sicht, das sollte deutlich geworden sein, sind die $\mathrm{Zu}$ schauerinnen und Zuschauer der Fixpunkt dieser Bezugnahme: ihr leiblichsinnliches Wahrnehmen; ihr Denken und Fühlen; die ästhetische Erfahrung, die sie in der Poiesis des Filme-Sehens in einem Wechselverhältnis mit dem jeweiligen audiovisuellen Medium für sich selbst gestalten oder machen. Letztlich ist es diese Zuschauerkonzeption, die den Anspruch begründet, dass Kunst, wie Kappelhoff schreibt, nicht verstanden werden darf „as an epiphenomenon of social reality, but as an aesthetic practice that is genuinely related to the political“. 84

Was genau bedeutet das? Im Rückgriff auf Rancières Konzepte der „Aufteilung des Sinnlichen“85 und des „Unvernehmens“86 führt Kappelhoff aus:

In the separate spaces of art Rancière sees a necessary prerequisite to giving language to the contingence of our world of the senses which allows for certain positions and relations without excluding others. In the spaces of separated sensibilities, art creates the possibility of such a dissensus without itself articulating it. It creates relations from which possibilities can emerge to speak from positions that are not provided for within the structure of sensuality in a given commonly shared world. ${ }^{87}$

Auch diese Überlegungen lassen sich auf eine Idee vom Zuschauer (und vom Zuschauen) beziehen. Zumindest dann, wenn man Rancière folgt, der - in einem, durchaus programmatisch, Der emanzipierte Zuschauer betitelten Essay - das Verhältnis von Theateraufführung und Theaterzuschauer mit jener von Lehrmeister und Schüler vergleicht, zugleich fordert, das pädagogische Verhältnis neu zu denken oder gar umzukehren:

Zuschauer zu sein ist nicht der passive Zustand, den wir in Aktivität umwandeln müssten. Es ist unsere normale Situation. Wir lernen und wir lehren, wir handeln und wir wissen

84 Hermann Kappelhoff: Poetics and Politics. In: ders.: The Politics and Poetics of Cinematic Realism. New York/Chichester 2015, S. 1-25, hier: S. 2. Bei der zitierten Monografie handelt es sich um eine Übersetzung von Kappelhoffs „Realismus“-Buch. Das Kapitel „Poetics and Politics“ ist jedoch nur in dieser Ausgabe zu finden, weshalb ich es in englischer Sprache zitiere.

85 „Aufteilung des Sinnlichen“ nenne ich jenes System sinnlicher Evidenzen, das zugleich die Existenz eines Gemeinsamen aufzeigt wie auch die Unterteilungen, durch die innerhalb dieses Gemeinsamen die jeweiligen Orte und Anteile bestimmt werden. Eine Aufteilung des Sinnlichen legt sowohl ein Gemeinsames, das geteilt wird, fest als auch Teile, die exklusiv bleiben. Diese Verteilung der Anteile und Orte beruht auf einer Aufteilung der Räume, Zeiten und Tätigkeiten, die die Art und Weise bestimmt, wie ein Gemeinsames sich der Teilhabe öffnet, und wie die einen und die anderen daran teilhaben." Vgl. Rancière: Die Aufteilung des Sinnlichen, S. 25-26.

86 Vgl. Jacques Rancière: Das Unvernehmen. Politik und Philosophie [1995]. Frankfurt a. M. 2002. 87 Kappelhoff: Poetics and Politics, S. 22. 
auch als Zuschauer, die in jedem Augenblick das, was sie sehen, mit dem verbinden, was sie gesehen und gesagt, gemacht und geträumt haben. Es gibt überall Ausgangspunkte, Kreuzungen und Knoten, die uns etwas Neues zu lernen erlauben, wenn wir erstens die radikale Distanz, zweitens die Verteilung der Rollen und drittens die Grenzen zwischen den Gebieten ablehnen. Wir müssen nicht die Zuschauer in Schauspieler/Akteure verwandeln und Unwissende in Gelehrte. Wir müssen das Wissen anerkennen, das im Unwissenden am Werk ist und die Aktivität, die dem Zuschauer eigen ist. Jeder Zuschauer ist bereits Akteur seiner Geschichte, jeder Schauspieler, jeder Mann der Tat ist der Zuschauer derselben Geschichte. ${ }^{88}$

Es geht Rancière also darum, „die etablierten Beziehungen zwischen Sehen, Machen und Sprechen neu zu formulieren“ “ ${ }^{89}$ Für ihn ist ebendies die Bedeutung des Wortes „Emanzipation“: „Das Verwischen der Grenzen zwischen denen, die handeln, und denen, die zusehen, zwischen Individuen und Gliedern eines Kollektivkörpers.“90

Ich denke, dass Kappelhoff einen sinnverwandten Begriff vom Zuschauer vertritt. In diesem Zusammenhang nimmt seine Vergleichsanalyse zwischen Leni Riefenstahls TAG DER FREIHEIT - UNSERE WEHRMACHT (D 1935) und Frank Capras PRELUDE TO WAR (USA 1942) eine paradigmatische Stellung ein. Sie ist auf die Einsicht gegründet, dass das, was wir Film nennen, letztlich nur „im Prozess des Verkoppelns von Bewegungsbild und Zuschauerkörper“ vorhanden ist; denn Film „ist nicht von dem Ereignis seines in Zuschauern sich verkörpernden Wahrnehmens, Fühlens und Denkens zu trennen"“. ${ }^{91}$ Darum also realisiert sich Film nur als Zuschauererfahrung; und darum kann Kappelhoff sagen, dass sich die Frage nach der Politik eines Films „auf der Ebene der ästhetischen Verfahren und Inszenierungsweisen entscheidet" ${ }^{92}$

In der Flucht dieses Gedankens will ich zwei, zugegebenermaßen recht pointierte, Thesen formulieren. Zum einen: So, wie der Film den Zuschauer denkt, so denkt er Politik. Zum anderen: In der ästhetischen Erfahrung gibt der Film den Zuschauerinnen und Zuschauern die Mittel an die Hand, die Verfasstheit des Gemeinwesens, dessen Teil sie sind, zu reflektieren, und ebenso ihren Platz innerhalb dieses Gemeinwesens. Audiovisuelle Bilder machen also historisch-politische Weltverhältnisse greifbar. Das heißt: Die Zuschauerinnen und

88 Jacques Rancière: Der emanzipierte Zuschauer [2008]. Wien 2009, S. 28.

89 Rancière: Der emanzipierte Zuschauer, S. 30 [Herv. i. O.].

90 Rancière: Der emanzipierte Zuschauer, S. 30.

91 Kappelhoff: Genre und Gemeinsinn, S. 75.

92 Kappelhoff: Genre und Gemeinsinn, S. 54. Es geht Kappelhoff hier um seine Deutung von Rortys „Sense of Commonality“; meines Erachtens ist es aber legitim, die zitierte Aussage auf den Politik-Begriff hin zu verallgemeinern. 
Zuschauer machen Politik, indem sie zuschauen - da das Schauen vom Handeln nämlich nicht zu trennen ist.

Jedenfalls steht fest, dass aus Kappelhoffs Perspektive die Rezeption audiovisueller Bilder immer interaktiv ist; immer geht es um eine Poiesis aufseiten der Rezipierenden, die als Herstellung jener Bilder begriffen werden muss. Das gilt für den Hollywood-Blockbuster, den obskuren Experimentalfilm, den abendlichen Fernsehkrimi, die Talkshow und das Musikvideo - nicht anders wie für Videospiele; auch für solche, wohlgemerkt, die, wie etwa DETROIT: BECOME HUMAN (Quantic Dream, 2018), einen hohen kinematografisch Anteil haben oder die Bedeutung des Gameplays zugunsten der Narration herunterschrauben, wie die Spiele des Entwicklers Telltale Games. So gesehen ist der Unterschied zwischen „Zuschauen“ und „Spielen“ vielleicht nicht derart schwerwiegend, dass man von kategorial unüberbrückbaren Divergenzen der Rezeptionssituation ausgehen müsste. ${ }^{93}$ Wenigstens ist das die Annahme, die den folgenden Analysen von Videospielen zugrunde liegt, welche dem Modus der Fantasy folgen.

\section{Zum Status der Analysen und zur Spielauswahl}

Die Politik eines Filmes findet also, mit Kappelhoff gesprochen, im verkörpernden Wahrnehmungsprozess der Zuschauerinnen und Zuschauer statt. ${ }^{94}$ Daraus folgt, dass die entscheidende Frage, wenn es um die politische Einschätzung audiovisueller Medien geht, darin besteht, welche Zuschauerposition der Film,

93 In diesem Zusammenhang sei noch einmal daran erinnert, dass auch Benjamin Beil die These vertritt, es sei durchaus angemessen die audiovisuellen Bilder von Film und Videospiel sowohl in theoretischer als auch in analytischer Perspektive vergleichend $\mathrm{zu}$ betrachten. Vgl. Beil: Genrekonzepte des Computerspiels, S. 15; vgl. auch: ders.: First Person Perspectives. Point of View und figurenzentrierte Erzählformen im Film und im Computerspiel. Münster 2010.

94 Mir scheint, dass Serjoscha Wiemer einen ähnlichen Ansatz verfolgt; „Videotechnologien“ stellen aus seiner Perspektive in erster Linie „Zeit- und Wahrnehmungstechnologien“ dar - es gehe hier um die „spezifische Abstimmung zeitlicher Relationen zwischen technischen Bildmedien und menschlicher Wahrnehmung“, wobei Wiemer die Zeit als Rohstoff versteht, „der moduliert und synthetisiert wird, um zeitliche Prozesse in Wahrnehmungsprozesse zu transformieren“. Denn: „Es ist nicht allein der Rezipient, der die Wahrnehmungsprozesse vollzieht, sondern die Videoapparatur selbst leistet Synthesen und Kontraktionen von Zeit." Serjoscha Wiemer: Videospiele als Zeitkristallisationsmaschinen. Aspekte einer temporalen Bildtheorie. In: Lars Grabbe/Patrick Rupert-Kruse/Norbert Schmitz (Hg.): Bildverstehen. Spielarten und Ausprägungen der Verarbeitung multimodaler Bildmedien. Marburg 2018, S. 136-161, hier: S. 137, S. 139 u. S. 140-141. 
die Fernsehserie, der YouTube-Clip, die Nachrichtensendung in der ästhetischen Erfahrung konstruiert. Oder eben: welche Spielerposition ein Videospiel hervorbringt. ${ }^{95}$

Wichtig ist freilich, dass dieser Ansatz nicht im Sinn eines Maßnahmenkatalogs oder einer Liste von Vorschriften verstanden werden darf. Was die Analyse leitet, ist zum einen die Poetik des jeweiligen Werks; zum anderen natürlich die konkrete Fragestellung. Es geht also weder um das Abhaken von Stichworten noch um die Wiederholung von theoretischen Formeln. Ganz im Gegenteil zielt die kappelhoffsche Methode darauf, die audiovisuellen Gestaltungsformen selbst als Modus des Denkens zu erfassen; darauf also, raumzeitliche Entwürfe zu erschließen, um im Nachvollzug ihrer Bauprinzipien die eigene Film- oder Spielerfahrung als interpersonell wirksames affektpoetisches Kalkül zu rekonstruieren, das wiederum genuin ästhetische Welt- und Selbstverhältnisse greifbar macht. Meines Erachtens erwächst der Film- oder Spielgenuss nicht zuletzt aus dem häufig unbewussten und immer spannungsreichen Prozess, der damit einhergeht, die in der ästhetischen Erfahrung projektierten Welt- und Selbstverhältnisse auf die im „Ich“ angelegten - und zumindest potenziell steter Wandelbarkeit unterworfenen - Welt- und Selbstverhältnisse zu beziehen. Wobei die so entstehende Dynamik wohl sehr viel mit dem Gefühl von Lebendigkeit zu tun hat, das die gelungene Kunsterfahrung bereitet.

Ich hoffe, dass die folgenden Analysen diesen grundlegenden Annahmen entsprechen. Jedenfalls ist es keineswegs meine Absicht, die Spiele als Anschauungsmaterial für vorgefertigte Theoreme $\mathrm{zu}$ verwenden. Das betrifft sowohl die Genrepoetik als auch die Analysemethode. Zwar bemühe ich mich, immer wieder deutlich zu machen - mal mehr, mal weniger explizit -, inwiefern die einzelnen Spiele grundlegende Bestandteile einer Poetik der Fantasy, so wie sie sich mir darstellt, in je eigenen poetologischen Entwürfen aufgreifen und verwirklichen. Doch sind die Analysen von den Spielen her gedacht, nicht von der Genrepoetik. Ähnliches lässt sich in Bezug auf die verwendete Methode sagen: Ich werde die Analysen nicht (oder nur ausnahmsweise) an Kappelhoffs Theorien oder Begrifflichkeit rückbinden. In der Art ihre Durchführung sollten die Analysen selbst deutlich machen, inwieweit sich ihre Ergebnisse einer bestimmten Vorgehensweise verdanken

\footnotetext{
95 Es stellt sich die Frage, wie eine solche Spielerkonzeption auf gängige Begrifflichkeiten der Game Studies bezogen werden kann, die das Verhältnis zwischen Spieler und Spielwelt theoretisch erfassen wollen, also etwa „Immersion“ oder „Involvement“. Diese Diskussion ist im Rahmen der vorliegenden Studie nicht zu leisten, weshalb ich auf eine systematische Nutzung der genannten Begriffe verzichte. Britta Neitzel bietet einen aktuellen Überblick zur „Immersions“- beziehungsweise „Involvement“-Diskussion; vgl. Neitzel: Involvement.
} 
(was umgekehrt nicht heißen soll, dass die Methodik für Fehlschlüsse meinerseits haftbar wäre).

Auch der Status der Analysen ist von Fall zu Fall verschieden. Bei SHADOw OF THE COLOSSUS und DARK SOULS habe ich mich an umfassenden poetologischen Rekonstruktionen versucht. Dass beide Analysen recht lang geraten sind, muss den Erfordernissen eines solchen Unterfangens zugeschrieben werden. Die Ausführlichkeit der Betrachtungen zu SHADOW OF THE COLOSSUS und DARK SOULS ist aber auch einem gewissen explorativen Ehrgeiz geschuldet: Ich wollte zum einen überprüfen, ob es überhaupt möglich ist, Videospiele in einer poetologischen Perspektive zu analysieren; zum anderen wollte ich herausfinden, auch in Hinblick auf den Stand der Game Studies, ob der Aufwand derartiger Analysen durch ihre Ergebnisse gerechtfertigt ist. Aus meiner Sicht sind beide Fragen $\mathrm{zu}$ bejahen.

Die restlichen Spielbetrachtungen der vorliegenden Studie würde ich eher als analytische Skizzen bezeichnen. Sie sind auf ein je spezifisches Erkenntnisinteresse hin durchgeführt und nehmen sich die Freiheit, selbst wesentliche Aspekte zu ignorieren, sofern ihr Verständnis nicht unbedingt vonnöten ist, um die Leitfrage(n) der entsprechenden Analyse zu beantworten; die Poetik der jeweiligen Spiele soll also nicht ignoriert oder instrumentell überformt, wohl aber in einem bestimmten, thetisch vorgeprägten Ausschnitt beleuchtet werden.

Im Fall von SKYRIM und THE BANNER SAGA geht es mir, wie bereits erläutert, um das Verhältnis zwischen Spieler und Spielwelt; die Analysen von THE LAST GUARDIAN, PYRE und HELLBLADE: SENUA'S SACRIFICE fokussieren die Frage, welche Funktion der agonalen bis martialischen Auseinandersetzung bei der Konstruktion einer Spielerposition zukommen kann; meine Überlegungen zu CONAN EXILES schließlich wollen überprüfen, ob und wie die „Poetik der Barbarei“, die so meine These - im Zentrum des Werkes von Robert E. Howard steht, sich im Medium des Videospiels zu verwirklichen vermag.

Vielleicht noch ein Wort zu der Spielauswahl. Zunächst habe ich mich auf Titel beschränkt, die in jüngerer Zeit erschienen sind. Dies aus mehreren Gründen: In Anbetracht der Tatsache, dass die ästhetische Wertigkeit des Genres Fantasy durchaus in Frage steht, schien es mir sinnvoll, Spiele zu analysieren, die vielleicht nicht repräsentativ, aber doch einigermaßen beispielhaft sind für das Entwicklungspotenzial dieses Genres im Medium Videospiel; Spiele also, die als ludische Kunstwerke gelten (vor allem SHADOW OF THE COLOSSUS), unverrückbare Bezugspunkte der Diskussion abgeben (vor allem SKYRIM und DARK SOULS) oder einfach sehr erfolgreich sind in ihrem jeweiligen Feld (CONAN EXILES als Multiplayer Online Survival-Game). Darüber hinaus wollte ich, im Rahmen des Tunlichen, auch eine gewisse Bandbreite von Spielentwürfen abdecken, 
nicht zuletzt, was die industrielle Stellung der jeweiligen Titel betrifft, weshalb ein Triple-A-Blockbuster wie SKYRIM ebenso vertreten ist wie PYRE und HELLBLADE, also verhältnismäßig unbekannte Independent-Titel. Schließlich habe ich mich bemüht, Spiele auszuwählen, die auf verschiedenen Plattformen verfügbar sind, um eine möglichst leichte Überprüfbarkeit meiner Ergebnisse zu gewährleisten - eine Ausnahme stellen hier die PlayStation-Exklusivtitel SHADOW OF THE COLOSSUS und THE LAST GUARDIAN dar, auf die ich allerdings aufgrund ihrer herausragenden künstlerischen Bedeutung nicht verzichten wollte.

Es versteht sich von selbst, dass meine Auswahl lückenhaft ist. Man hätte zweifellos obskurere Fantasy-Spiele finden können als PYRE, und es gibt ganze Spieltypen oder Spielgenres, die ich nicht berücksichtigt habe, etwa die JRPG (also die Japanese role-playing games, die sich unter anderem durch eine bestimmte, vom Manga beziehungsweise dem Anime inspirierte Ästhetik auszeichnen). Vor allem finde ich bedauerlich, dass es mir nicht möglich war, Spiele wie ELEX und HORIZON ZERO DAWN ausführlicher zu berücksichtigen, da sie in ihren Weltentwürfen archaische und futuristische Zeitlichkeiten kombinieren und somit einen ganz eigenen, noch weitestgehend unverstandenen Modus der Fantasy gestalten.

Aber es ist wohl wenig sinnvoll, eine lange Liste der Versäumnisse zu erstellen; vielmehr hoffe ich, dass die Analysen, welche die vorliegende Studie strukturieren, in ihrem Zusammenwirken greifbar machen, was die besonderen Möglichkeiten des Mediums Videospiel sind, wenn es um die Fantasy geht.

\section{Die Freiheit der „Abenteuerzeit“: THE ELDER SCROLLS V: SKYRIM}

Die ELDER-SCROLLS-Spiele zielen darauf ab, wie Markus Engelns feststellt, „dem Spieler größtmögliche Freiheiten in der Gestaltung des Spielprozesses“ zu gewähren. ${ }^{96}$ Das gilt bereits für THE ELDER SCROLLS II: DAGGERFALL (Bethesda Softworks, 1996); es gilt ebenso für die Nachfolger THE ELDER SCROLLS III: MORROWIND (Bethesda Game Studios, 2002), THE ELDER SCROLLS IV: OBLIVION (Bethesda Game Studios, 2006) und THE ELDER SCROLLS V: SKYRIM. Vielleicht könnte man sagen, dass dieses Ansinnen den Kern der Poetik jener Spielereihe bildet. Wenn dem so ist, muss die Freiheit der Spielerinnen und Spieler sich unmittelbar

96 Markus Engelns: Vom Helden zum Mörder? Zur Herstellung einer „Atmosphäre des Mordens“ in THE ELDER SCROLLS V - SKYRIM. In: Christian Huberts/Sebastian Standke (Hg.): Zwischen|Welten. Glückstadt 2014, S. 117-152, hier: S. 133. 
niederschlagen in der Ausgestaltung der Spielmechaniken und dem Gepräge der Spielwelt. ${ }^{97}$ Und da die Freiheit des Spielers letztlich eine Freiheit gegenüber dem Spiel ist, muss sich, wenn Engelns recht hat, in den ELDER-SCROLLSSpielen auf exemplarische Weise eine bestimmte Idee der ludischen Fantasy realisieren. Jene nämlich, dass die Poiesis des Videospiele-Spielens, sofern es um dieses Genre geht, zunächst und vor allem eine Poiesis des Welt-Erlebens, des Welt-Genießens und sogar des Welt-Gestaltens ist. Die Spielwelt mag, wenn man so sagen kann, als digitales Reservoir vorhanden sein, aber sie ist nicht zuhanden; indessen erhält sie ihre Einheitlichkeit - als je einmalige und unwiederholbare Realisierung der in ihr angelegten ästhetischen Gesetzmäßigkeiten - erst in der Spielerfahrung, die eben kein passives Widerfahrnis ist, sondern etwas überaus Aktives; im kappelhoffschen Sinn ein Machen der Spielerinnen und Spieler.

Wir wollen sehen, ob sich diese These aufrechterhalten lässt, und was sie hinsichtlich des fünften Teils der ELDER-SCROLLS-Reihe bedeuten mag. Da trifft es sich, dass SKYRIM zu jenen Spielen zählt, die in der Forschung gerne herangezogen werden, wenn das Genre Rollenspiel, die ludische Fantasy oder die weltenbauenden Kompetenzen des Mediums Videospiel in Rede stehen. „A key function of ludic agency is the player's ability to have embodied interactions in the game space“, ${ }^{98}$ erklärt Lindsay Joyce - und offenbar ist SKYRIM ein Spiel,

97 Im Folgenden konzentriere ich mich, wie gesagt, auf das Verhältnis von Spieler und Spielwelt. Allerdings könnte man ebenso gut andere Aspekte des Spieldesigns heranziehen, um zu verdeutlichen, inwiefern SKYRIM nach Maßgabe der größtmöglichen Spielerfreiheit gestaltet ist. Franziska Ascher merkt etwa an, dass SKYRIM keine festgesetzten Klassen mit bestimmten Vorteilen und Nachteilen zur Auswahl stellt (was ihrer Meinung nach auf „einen Bruch mit herkömmlichen Rollenspielkonzepten“ hinausläuft), sondern den Spielerinnen und Spielern die Möglichkeit gibt, den Avatar in einer überaus vielfältigen Kombinatorik zu entwickeln. Franziska Ascher: Heldenkarrieren. Der SKYRIM-Avatar und sein narratives Potential. In: Paidia. Zeitschrift für Computerspielforschung, 07. Mai 2012, http://www.paidia.de/heldenkarrieren/ [letzter Zugriff: 12.05.2020].

Hier ist ein Verweis auf die Forschung von Casey Hart am Platz, der mit empirischen Methoden untersucht hat, welches Verhältnis Spielerinnen und Spieler zu einem von ihnen gestalteten Avatar einnehmen. Harts Studie basiert auf SKYRIM, und wenn er zu dem Schluss kommt, „that players may be more likely to experiment with alternate self constructs in most aspects of personality, than to build and play avatars according to either actual self or ideal self constructs“, so erhebt sich die Frage, inwiefern die Freiheiten, die die ELDER-SCROLLS-Reihe gewährt, eine solche Spielerhaltung zumindest begünstigen - obgleich Harts Resultate auch in einer verallgemeinernden Perspektive plausibel erscheinen. Casey Hart: Getting into the Game. An Examination of Player Personality Projection in Videogame Avatars. In: Game Studies 17 (2017), H. 2, http://gamestudies.org/1702/articles/hart.

98 Lindsey Joyce: KENTUCKY ROUTE ZERO. Or, How Not to Get Lost in the Branching Narrative System. In: Matthew Wilhelm Kapell (Hg.): The Play Versus Story Divide in Game Studies. Critical Essays. Jefferson 2016, S. 17-27, hier: S. 20. 
das diesen Umstand evident macht. Der Konnex zwischen Spielwelt und Avatar scheint dabei tatsächlich in einem Entwurf spielerischer Freiheit oder freiheitlichem Spielgenuss zu bestehen. Ebenso schlicht wie treffend heißt es bei Jonne Arjoranta: „Walking around the game world is an adventure, and the game encourages bold exploration. “99 Bezogen auf Bethesdas Fantasy-Rollenspiele im Allgemeinen und SKYRIM im Speziellen formuliert Dan Whitehead einen ähnlichen Gedanken: „A huge part of the appeal of the ELDER SCROLLS series is the knowledge that you can set out in any direction and find something of interest.“100 Oder noch einmal anders: „Bethesda RPGs are more about place than plot. They're about roaming, poking around and seeing what happens, and the various quest lines are there to tug you in the direction of new places. “101

Die Spielerfreiheit erweist sich in SKYRIM (beziehungsweise der ELDERSCROLLS-Reihe) also zunächst als eine Freiheit der Bewegung. Die Bewegungsfreiheit ist dabei an ein Versprechen geknüpft. Es ist das Versprechen an die Spielerinnen und Spieler, um Whitehead zu paraphrasieren, dass sie in jeder Himmelsrichtung, bei Sonnenschein und Schneesturm, im Wald und am Hügelhang, auf den höchsten Gipfeln und tief unter der Erde, in Ansammlungen ärmlicher Hütten ebenso wie in stolzen Städten, eine Geschichte, ein Abenteuer, kurzum: „something of interest“ finden werden.

Einmal mehr begegnen wir hier einer Reformulierung der Verheißung mythopoetischer Welten. Was für eine Welt aber ist es, in der wir uns bewegen, wenn wir SKYRIM spielen? Robert Baumgartner erläutert, welches Konzept der Welt des Spiels zugrunde liegt, indem er die Unterschiede zwischen DAGGERFALL, dem zweiten Teil der ELDER-SCROLLS-Reihe, und seinen Nachfolgern - MORROWIND, OBLIVION und SKYRIM - herausarbeitet:

Wie ihr Vorgänger DAGGERFALL bieten sie umfangreiche dreidimensionale Spielwelten, die mit Städten, Dörfern, Verliesen, Festungen, Ruinen, Bürgern, Monstern und umfangreicher Flora und Fauna ausgestattet sind. Der Vergleich mit DAGGERFALL eröffnet jedoch zugleich die erste Eigenheit: Im Gegensatz zum gemeinsamen Vorgänger sind die ,world maps' trotz ihrer augenscheinlichen Größe erstaunlich klein: Die in MORROwIND geschaf-

99 Jonne Arjoranta: Narrative Tools for Games. Focalization, Granularity, and the Mode of Narration in Games. In: Games and Culture 12 (2017), H. 7-8, S. 696-717, hier: S. 712.

100 Dan Whitehead: THE ELDER SCROLLS V: SKYRIM - DRAGONBORN review. We're off to see the lizard. In: www.eurogamer.net, 07. Dezember 2012, https://www.eurogamer.net/articles/201212-05-the-elder-scrolls-5-skyrim-dragonborn-review [letzter Zugriff: 12.05.2020; Herv. i. 0.].

101 Dan Whitehead: THE ELDER SCROLLS V: SKYRIM - DAWNGUARD review. Morning glory? In: www.eurogamer.net, 27. Juni 2012, https://www.eurogamer.net/articles/2012-06-27-the-elderscrolls-5-skyrim-dawnguard-review [letzter Zugriff: 12.05.2020]. 
fene Provinz von Vvardenfell besitzt (in reale Flächeneinheiten umgerechnet) eine Fläche von 24 Quadratkilometern, die Provinz Cyrodiil aus oBLIvion eine Fläche von ca. 41 Quadratkilometern, während die reine Oberflächenwelt von SKYRIM auf eine Fläche von ca. 37 Quadratkilometer [sic] kommt - dazu muss noch berücksichtigt werden, dass davon bis zu einem Viertel als Berggipfel oder Levelgrenzen nicht von Spielern betreten werden können. ${ }^{102}$

Baumgartner stellt weiterhin fest, dass diese Maße verglichen mit jenen der Spielwelt von DAGGERFALL, die „fast 500.000 Quadratkilometer“ umfasst, „freilich winzig“ wirken, betont aber im selben Moment, dass die „reine Ausdehnung kein Ausschlusskriterium für eine atmosphärisch, gut' oder ,schlecht' arbeitende Spielwelt“ sein könne. ${ }^{103}$

DAGGERFALL kann jene „räumliche Expansion“ nämlich nur um den Preis „der Austauschbarkeit der meisten Orte“ erzielen: „Aufgrund des nicht anders zu bewältigenden Arbeitsaufwandes sind fast alle Dungeons, Städte und Bewohner von DAGGERFALL aus einem kleinen Inventar an Schablonen zufallsgeneriert und unterscheiden sich oft nur in wenigen Details voneinander.“104 Bezogen auf die Welterfahrung hat diese „Austauschbarkeit“ den unerfreulichen Effekt, dass man „nur scheinbar“ von Ort zu Ort reist, da den einzelnen Lokalitäten eben das unverwechselbare Gepräge, oder, wie Baumgartner mit Gernot Böhme sagt, die „Atmosphäre“ fehlt. ${ }^{105}$ Die Entwickler von Bethesda haben die latente Beliebigkeit der Spielwelt von DAGGERFALL offenbar als Problem erkannt; jedenfalls sind die drei Folgespiele der ELDER-SCROLLS-Reihe, was die Weltgestaltung betrifft, dadurch gekennzeichnet, dass sie „die expansive Raumstrategie ihres Vorgängers massiv abgewandelt haben“. ${ }^{106}$

102 Robert Baumgartner: Ankunftsmomente. Die Vermittlung von Atmosphäre in Rollenspielen am Beispiel der ELDER SCROLLs-Reihe. In: Huberts/Standke (Hg.): Zwischen/Welten, S. 71113, hier: S. 84. Übrigens geht Baumgartner von einer ähnlichen These aus wie ich, betont er doch, dass die Räume des Videospiels „nicht nur als narratologisch vermittelte Zeichenkonstrukte“ existieren, „sondern (besonders nach der Einführung leistungsfähiger Hardware) auch als interaktive dreidimensionale Raumsimulationen, die von Spielern - vermittelt durch den Avatar - erkundet und beeinflusst werden können“. Baumgartner: Ankunftsmomente, S. 72.

103 Baumgartner: Ankunftsmomente, S. 84.

104 Baumgartner: Ankunftsmomente, S. 79-80.

105 Vgl. Baumgartner: Ankunftsmomente, S. 81. In dem zitierten Aufsatz legt Baumgartner eine kritische Lesart von Böhmes Atmosphärenbegriff vor, den er durchaus produktiv auf das Medium Videospiel bezieht. Vgl. Gernot Böhme: Aisthetik. Vorlesungen über Ästhetik als allgemeine Wahrnehmungslehre. München 2001. Vgl. auch: ders.: Atmosphäre. Essays zur neuen Ästhetik. Berlin 2013.

106 Baumgartner: Ankunftsmomente, S. 84. 
Baumgartner fährt fort zu erläutern, dass es im Design von DAGGERFALL die „erkennbare Anstrengung“ gegeben habe, „eine fiktionale Fantasywelt primär durch das Faktum ihrer im Vergleich zur Realität maßstabsgetreuen Abbildung als ,Territorium‘, als Raumeinheit mit einer ,realistischen“ Ausdehnung und Bevölkerung, als glaubwürdig darzustellen“. ${ }^{107}$ Im Gegensatz dazu streben MORROWIND, OBLIVION und SKYRIM danach, ihre vergleichsweise kleinen, handgestalteten Welten mit möglichst vielen Örtlichkeiten zu füllen, die ein individuelles, unverwechselbares Gepräge aufweisen, ${ }^{108}$ sodass die entsprechenden Provinzen - Vvardenfell, Cyrodiil oder eben Skyrim -, sich anfühlen wie über viele Jahrzehnte oder gar Jahrhunderte aus der Tiefe einer halb in mythischem Nebel verborgenen Geschichtlichkeit erwachsene Topografien, die Krieg und Frieden, Epochen des Wohlstands ebenso wie bittere Not und Seuchen erlebt haben. Und die, in ihrer historischen, klimatischen und geologischen Bedingtheit, eine Heimat für verschiedene Rassen und Spezies, natürliche und übernatürliche Geschöpfe darstellen, die sehr verschiedenen Lebensweisen anhängen, unterschiedlichste Kulte und Bräuche pflegen - so die Entwicklungsgesetze jener Welten sie in den Stand gebracht haben, dergleichen zu tun -, und denen gar keine Wahl bleibt, als eine oftmals prekäre Nachbarschaft zu pflegen.

In THE ELDER SCROLLS III-V ist diese Nachbarschaft insofern besonders prekär, als die Welten der drei Spiele „bis zur Grenze der Glaubwürdigkeit mit Monumenten und erkundbaren Orten“" gefüllt sind. ${ }^{109}$

Baumgartner führt aus:

Jede der Welten beherbergt Dutzende von (Klein-)Städten und Dörfern und zahlreiche erforschbare Dungeons in Form von verlassenen Minen und Festungen, Schiffswracks, Ruinen, verfallenen Schreinen, Mausoleen und Höhlen. SKYRIM allein enthält 186 solcher Dungeons, ergo mindestens fünf Festungen/Minen/Banditenlager etc. pro Quadratkilometer. Zusammen mit Städten, Gehöften, Armeelagern und anderen ,zivilisierten“ Orten sowie natürlichen Raumkonstellationen wie Seen, Wasserfällen oder Bergen erscheinen die Welten der drei Titel damit bis zum Bersten gefüllt mit Orten und Objekten. ${ }^{110}$

Die Spielerinnen und Spieler wandern also „durch eine Landschaft, die scheinbar aus sonst in ihrer Seltenheit einmaligen Naturwundern und historischen Artefakten zusammengesetzt ist“; und dasselbe Prinzip, so Baumgartner weiter, liege „der klimatischen und biologischen Diversität der extrem kleinen Flächen“ zugrunde:

107 Baumgartner: Ankunftsmomente, S. 84.

108 Vgl. Baumgartner: Ankunftsmomente, S. 85.

109 Baumgartner: Ankunftsmomente, S. 85.

110 Baumgartner: Ankunftsmomente, S. 85-86. 
so wird in SKYRIM Taiga nach wenigen Kilometern von dunklen Nadelwäldern ersetzt, welche wiederum bald darauf herbstlichen Birkenhainen und sanften Hügeln weichen. In der Realität jedoch sind diese Übergangszonen meist hunderte von Kilometern voneinander entfernt. ${ }^{111}$

Zusammengenommen erlauben es Baumgartners Anmerkungen, die der Weltkonstruktion von SKYRIM (wie wohl auch von MORROWIND und OBLIVION) zugrundeliegende Idee von Spielerfreiheit zu bestimmen. Sie rührt daher, dass die Spielerinnen und Spieler keine Entscheidung treffen müssen; oder, vielleicht richtiger gesagt, daher, dass die unendlich vielen Entscheidungen, die sie im Lauf der Zeit treffen, sich nicht zu einer unwiderruflichen Grundentscheidung summieren: Wer nach Süden geht, kann jederzeit umdrehen, um nach Norden zu gehen; wem ein Dungeon zu schwer erscheint, kann ihn jederzeit verlassen, um später wieder zurückzukehren und sich einstweilen dem Banditenlager im angrenzenden Sumpf oder dem verfallenen Turm auf der nahen Hügelkuppe zuwenden. Für gewöhnlich ändert sich weder etwas an der Zugänglichkeit der Orte noch an dem, was sie für die Spielerinnen und Spieler bereithalten. ${ }^{112}$

Ein ähnliches Prinzip bestimmt die Zeitlichkeit der Erkundung: Auch abgesehen von der Schnellreisefunktion, die es ermöglicht, einmal entdeckte Orte von jedem (im Freien gelegenen) Punkt der Karte in Sekundenschnelle (beziehungsweise der Dauer eines Ladebildschirms) zu erreichen, sind die Entfernungen in der Provinz Skyrim - sozusagen dem skandinavischen Zipfel des Fantasy-Kontinents Tamriel - nicht solcherart gehalten, dass die Entscheidung, beispielsweise das Gebiet um die an der Nordküste gelegene Stadt Dawnstar zu bereisen, nicht jederzeit wieder rückgängig gemacht werden könnte, wenn man plötzlich zu dem Schluss kommt, dass man doch lieber zuerst Riften in der südöstlichen Ecke der Karte besuchen möchte.

Ganz allgemein kann es - aufgrund der von Baumgartner beschriebenen Verdichtung in der Weltgestaltung - leicht geschehen, dass die Spielerinnen und Spieler ihren Abenteuern eine Zeitlichkeit angedeihen lassen, die im permanenten Treffen und Revidieren von Entscheidungen besteht: Unterwegs zu einem Grabmal, in dem ein wertvolles Schwert verborgen sein soll, stoßen sie auf eine einsame Waldeshütte, die sie unwiderstehlich anzieht. Dann mag es sich so ergeben, dass sie in besagter Hütte den Durchgang zu einem weitläufigen, höhlenartigen Diebesversteck entdecken. Und wenn sie schließlich

111 Baumgartner: Ankunftsmomente, S. 86.

112 Freilich gibt es Orte, die im Lauf der Handlung zerstört werden können, wie etwa das Versteck der Dark Brotherhood in der Nähe von Falkreath, oder nur im Rahmen von bestimmten Questen erreichbar sind, etwa die hoch in den Velothi Mountains gelegenen Ruinen des Drachentempels von Skuldafn. 
sämtliche Diebe ins Jenseits befördert und alle Winkel des Verstecks erkundet haben, sind sie vielleicht derart schwer beladen mit Waffen, Rüstungsteilen, Tränken, Büchern, Schriftrollen und Schmuckstücken, dass sie sich entschließen, erstmal die nächste Stadt aufzusuchen, um einen Teil der Beute zu verkaufen. In der örtlichen Schenke geraten sie dann zufällig in ein Gespräch mit einem Fremden, der sie zu einem Trinkwettbewerb auffordert und plötzlich verlieren sie das Bewusstsein und finden sich viele Meilen entfernt an einem Ort wieder, den sie nie zuvor gesehen haben ... Möglicherweise wird sich ein Spieler, dem dergleichen geschieht, erst ein Dutzend Stunden später an dieses wertvolle und mächtige Schwert erinnern, das er doch längst in seinen Besitz hatte bringen wollen.

Mir scheint, dass die Zeitlichkeit, die SKYRIM gestaltet - beziehungsweise der Modus von Zeitlichkeit, den das Spiel den Spielerinnen und Spielern zur Gestaltung anbietet -, dem Chronotopos der „Abenteuerzeit“ ähnelt. Zuvörderst am antiken Roman, etwa Leukippe und Kleitophon von Achilleus Tatios (2. Jhd. n. Chr.), entwickelt Bachtin die „Abenteuerzeit“, und „in ihrem Inneren“ ist sie seines Erachtens wie folgt beschaffen:

\begin{abstract}
„Plötzlich“ und „gerade“ - diese Wörter charakterisieren am besten diese ganze Abenteuerzeit, die ja allgemein dort beginnt und in ihre Rechte tritt, wo der normale und pragmatisch oder kausal begriffene Gang der Ereignisse abreißt und der reinen Zufälligkeit mit ihrer spezifischen Logik Platz macht. Diese Logik besteht in einer zufälligen Kongruenz, d. h. in einer zufälligen Gleichzeitigkeit, und in einer zufälligen Inkongruenz, d. h. einer zufälligen Ungleichzeitigkeit. Hierbei ist auch das „Früher“ oder „Später“ dieser zufälligen Gleichzeitigkeit und Ungleichzeitigkeit von grundlegender und entscheidender Bedeutung. Geschähe irgendetwas nur eine Minute früher oder später, d.h., wäre eine bestimmte zufällige Gleichzeitigkeit oder Ungleichzeitigkeit nicht vorhanden, so ergäbe sich auch keinerlei Sujet, und der Roman wäre gegenstandslos. ${ }^{113}$
\end{abstract}

In SKYRIM lässt sich namentlich das Verhältnis zwischen Spieler und Spielwelt, was die Dynamik der Erkundung betrifft, als „Abenteuerzeit“ beschreiben. Um bei dem skizzierten Beispiel zu bleiben: Vielleicht hätten wir uns gar nicht für die Hütte im Wald interessiert, wenn wir nicht des Abends an ihr vorbeigekommen wären, sie also im idyllischen und zugleich geheimnisvollen Dämmerlicht der sinkenden Sonne erblickt hätten. Und vielleicht wären wir nie auf den Gedanken gekommen, uns auf die Zecherei einzulassen, wenn wir nicht den Eindruck gehabt hätten, dass unser Held oder unsere Heldin nach erfolgreichem Verkauf einiger Beutestücke in Feierlaune sein könnte.

113 Michail M. Bachtin: Chronotopos [1975]. Frankfurt a. M. 1986, S. 15 [Herv. i. O.]. 
Weil man in SKYRIM stets die Freiheit hat, zwischen Dutzenden verschiedener Handlungsoptionen auszuwählen, gibt es eine Willkür und Zufälligkeit in allem, was die Spielerinnen und Spieler tun. Zugleich brauchen diese Willkür und Zufälligkeit eine bestimmte Taktung, müssen im Rhythmus des „Plötzlich!“ und „Gerade!“ sich ereignen, damit Geschichten, also Verwicklungen und Verstrebungen von Ereignissen, zustande kommen. Es ist der Zufälligkeit willkürlicher Entscheidungen geschuldet, dass dieser Spieler oder jene Spielerin die Welt von SKYRIM in ihrem Erleben so und nicht anders ausgestaltet, doch bedarf diese Zufälligkeit der Präzision des Ungewissen und Unplanbaren, die im Einschwingen auf einen audiovisuellen Rhythmus hervortritt, welcher wiederum im Zusammenwirken zwischen Spielwelt und Spieler sich herausbildet. Versteht man den Chronotopos, nach Bachtins sicher etwas unbefriedigender Definition, als den „grundlegenden wechselseitigen Zusammenhang“ von „künstlerisch erfaßten Zeit-und-Raum-Beziehungen“, ${ }^{114}$ so kann man sagen, dass die „Abenteuerzeit“ eine Potenzialität der Spielerfahrung von SKYRIM bezeichnet, die zu ihrer Realisierung freilich auf die Bereitschaft der Spielerinnen und Spieler angewiesen ist, die Welt im beschriebenen Sinn zu gestalten.

Das wäre also eine Art, die Freiheit zu beschreiben, welche damit einhergeht, dass sich die Verheißung der mythopoetischen Welt im Medium Videospiel erfüllt: Es ist eine Freiheit, die bestimmte Konzepte, eine Welt zu gestalten, einen Avatar zu entwerfen, eine Geschichte zu erzählen, in einer Idee von Bewegung zusammenschließt.

Zugleich jedoch gibt uns die ELDER-SCROLLS-Reihe auch die Freiheit, jene Potenzialität ungenutzt zu lassen. Mir scheint, dass sich namentlich die Schnellreisefunktion als falscher Freund erweisen kann. Selbstredend wird sie fast jeder Spieler früher oder später nutzen, um Zeit zu sparen. Nutzt man sie aber zu häufig, verlässt sich gar ausschließlich auf die Möglichkeit, innerhalb weniger Augenblicke von einem Ende der Karte ans andere zu gelangen, so löst sich die Spielwelt in Abstraktionen auf, und der Spielprozess selbst wird zu einer Folge von Erledigungen und abzuhakenden Aufgaben. Dann gibt es kein Zusammenwirken einer „zufälligen Kongruenz“ mit einer „zufälligen Inkongruenz“ mehr, sondern nur noch die Linearität einer mechanistischen Erfüllungslogik, welche die Unberechenbarkeit der paradoxen und aufregenden „Abenteuerzeit“ in eine Art neoliberales Selbstoptimierungsprogramm für Helden und angehende Weltretter verkehrt, die immer noch eine Queste mehr erledigen, noch eine Stufe höher steigen wollen, und eine derartige Progression als Letztbegründung ihres Spielens begreifen.

114 Bachtin: Chronotopos, S. 7. 
Ich will nicht sagen, dass ein solches Spielen ,verfehlt‘ ist. In seiner Anlage hält SKYRIM eine Vielzahl konkurriender Möglichkeiten bereit, die Spielerfahrung zu gestalten. Allerdings scheint mir, dass das Spiel seine Poetik nur dann vollgültig erfüllen kann, wenn die Spielerinnen und Spieler ganz bestimmte und sehr schlichte - „embodied interactions in the game space“ vollziehen: Es gilt, die Spielwelt zu durchwandern (oder zu durchreiten) und dabei, immer wieder aufs Neue, dem Rhythmus der „Abenteuerzeit“ als einem Modus der Welterschließung den Takt zu geben.

Was aber sind die Grenzen der Welt, die man sich solcherart erschließt? Anders gesagt: Nutzen sich die Möglichkeiten, in einer digitalen Provinz von weniger als 40 Quadratkilometern die Freiheit der „Abenteuerzeit“ zu realisieren, nicht allzu schnell ab? Tatsächlich erkennt Paul Martin das Problem der ELDER-SCROLLSReihe darin, dass die Spiele, in den grundlegenden Bauprinzipien ihrer Welten, ein Versprechen machen, das sie letztlich nicht erfüllen können.

Er schreibt:

Once we have put in enough hours to have reached the game's final cut-scene we have encountered the invisible walls of the oceans and mountains - Tamriel is bounded and we have seen the boundaries. We have noted the repetitions involved in the design of dungeons. The minor cities have been comprehended as uniform content - guilds, shops, residences, cathedral and castle - with variations in arrangement and architecture. The plains of OBLIVION have been understood as a set of half a dozen repeating worlds. In short, the awesome breadth of Tamriel has been transformed over the course of the game to a set of discrete, manageable spaces. Unlike in comparable epic films, in which each landscape shot presents as the sublime, once the player is allowed to walk the fields and outside the sublimely framed shot - the sense of grandeur can no longer be sustained, or at least it can only be sustained fitfully. ${ }^{115}$

Zwar beziehen sich diese Ausführungen zuvörderst auf OBLIVION, den vierten Teil der Reihe, doch lässt sich Martins Kritik ohne weiteres auf MORROWIND oder SKYRIM übertragen. In dieser Perspektive kann man also sagen: Schneller, als es uns lieb ist, entpuppt sich die vermeintliche Grenzenlosigkeit der Spielwelt als Illusion, und die erhabenen Landschaften von Vvardenfell, Cyrodiil oder Skryim schrumpfen auf die Maße eines wohlgepflegten Schrebergartens zusammen - und erscheinen dann auch ebenso aufregend und gefährlich wie ein solches Gärtchen. ${ }^{116}$

115 Paul Martin: The Pastoral and the Sublime in ELDER SCROLLS IV: OBLIVION. In: Game Studies 11 (2011), H. 3, http://gamestudies.org/1103/articles/martin.

116 Tatsächlich greift Martin selbst zu diesem Bild. Über die Spielwelt von oBLIVION schreibt er: „It does not extend beyond the horizon but is bounded on all sides. It is not as you find it, but carefully arranged and ordered. It is not, like the wilderness, a sublime chaos, but, like the garden, a picturesque design." Martin: The Pastoral and the Sublime in ELDER SCROLLS IV: OBLIVION. 
Diese Kritik hat sicherlich etwas für sich. Zugleich jedoch ist sie auf Voraussetzungen gegründet, die man nicht unbedingt teilen muss. Und das in mehrfacher Hinsicht. So scheint mir keineswegs ausgemacht, dass der Genuss einer Fantasy-Spielwelt notwendigerweise an die Grandezza der Überwältigungsästhetik gebunden ist. Als Modus der Spielerfahrung betrachtet, liegt etwas durchaus Gewöhnliches in der „Abenteuerzeit“; es ist die Alltäglichkeit des Heldendaseins, die darauf hofft, dass sich in der Fülle beliebiger Entscheidungen die Wirkmacht des „Jetzt!“ und des „Gerade!“ realisiert. Dass dergleichen sich zuträgt, ist eben weniger an bestimmte Raum-Qualitäten gebunden (etwa die endlose Weite einer unerforschten Ebene), als vielmehr an Zeit-Qualitäten, in denen sich der Raum auf eine bestimmte Weise erschließt, weshalb die Verheißung der Mythopoesie in (beispielsweise) dem fünfzigsten verfallenen Turm eine Aktualisierung erfahren kann. ${ }^{117}$ Der Turm mag von Dieben oder Nekromanten bewohnt sein; vielleicht ist er auch einfach leer - der Zauber besteht nicht in der Großartigkeit der zu enthüllenden Geschichte oder dem Spektakulären der Erkundung, sondern darin, dass diese oder jene Handlung, in aller zufälligen Notwendigkeit, an diesen oder jenen zufällig-notwendigen Ort geführt hat. Das Versprechen der „Abenteuerzeit“ besteht also im Rhythmus der Welterkundung selbst. Vielleicht kann die Erkundung dieses von ihr selbst gegebene Versprechen nicht immer einlösen; es wird aber eben auch nicht gebrochen, sondern erneuert sich gleichsam auf Schritt und Tritt.

Abgesehen davon betrachtet Martin die Welten der ELDER-SCROLLS-Reihe in einer sehr eingeschränkten Perspektive: nämlich als das, was sie faktisch darstellen. Die quantifizierbaren Ausmaße einer Spielwelt sind indessen, es sei noch einmal unterstrichen, nicht mit ihren Erfahrungspotenzialen gleichzusetzen. Zweifellos rührt die Sehnsucht nach dem Horizont daher, dass er, ebenso wie das Ende des Regenbogens, weder erreicht noch berührt noch erschlossen werden kann. Aber das heißt doch auch, dass diese Sehnsucht gerade aus der Erfahrung einer ebenso unsichtbaren wie unüberschreitbaren Grenze erwächst;

117 Marc Bonner bringt ein vergleichbares Argument vor, wenn er - unter Bezugnahme auf die von Deleuze und Guattari entwickelten Kategorien des „glatten“ und des „gekerbten“ Raumes - schreibt: „Dabei ist die Modularität und Variabilität der gekerbten Wildnis, ihr Rhythmus in Form der linearen und zyklischen Wiederholungen ausschlaggebend für die Illusion von Weite, Wildheit und Freiheit.“ Marc Bonner: Die gekerbte Wildnis. Inszenierungen vermeintlich unberührter Umwelt in digitalen Spielwelten. In: Paidia. Zeitschrift für Computerspielforschung, 28. Februar 2018, http://www.paidia.de/die-gekerbte-wildnis-inszenierungen-ver meintlich-unberuehrter-umwelt-in-digitalen-spielwelten/ [letzter Zugriff: 12.05.2020]. Vgl. auch: Gilles Deleuze/Félix Guattari: 1440. Das Glatte und das Gekerbte [1980]. In: Jörg Dünne/Stephan Günzel (Hg.): Raumtheorie. Grundlagentexte aus Philosophie und Kulturwissenschaft. Frankfurt a. M. 2006, S. 434-445. 
was jenseits dieser Grenze liegt, ist und bleibt unverfügbar. Anders gesagt: Die Limitationen der Spielwelt selbst können ihr Gegenteil, die Unermesslichkeit, in sich schließen und ahnungsweise erfahrbar machen.

Franziska Ascher gibt ein schönes Beispiel dafür, was das bezogen auf SKYRIM bedeutet:

Während ihres Umherstreifens loten die Spieler gerne auch einmal die Grenzen der Spielwelt aus, was typisch für Paidia ist. Was aber wollen sie da, wo laut Karte die Welt zu Ende ist? Geht es ihnen darum, das graue Nichts jenseits der Spielwelt zu erblicken und damit einen End-of-the-World-Schock zu provozieren? Keineswegs - sie versuchen einen Blick auf die Länder Morrowind, Cyrodiil oder Hammerfell zu erhaschen, die Schauplätze frühere [sic] ELDER SCROLLS-Titel waren. Und die Entwickler geben sich keine Blöße: Man sieht ihn tatsächlich, wenn man von der richtigen Stelle aus nach Osten schaut - den großen Vulkan Vvardenfell, der im roten Jahr Morrowind verwüstete und tausende Dunkelelfen zur Flucht nach Himmelsrand zwang. Hier geht es ganz offensichtlich nicht darum, ein paar schlecht aufgelöste graue Zacken zu sehen - hier geht es um die Intertexte und die Narration von SKYRIM. Genannte Zacken sind nämlich derart narrativ aufgeladen, dass ihr konkretes Aussehen vollkommen nebensächlich wird. ${ }^{118}$

Wobei nicht nur die grafische Güte der grauen Zacken „vollkommen nebensächlich“ ist; vielleicht kommt es nicht einmal darauf an, ob man tatsächlich andere ELDER-SCROLLS-Titel gespielt hat und also weiß, was der Vulkan Vvardenfell intertextuell oder narrativ zu bedeuten haben mag. Die schiere Tatsache, dass da etwas jenseits der Spielwelt aufscheint, was zugleich als Teil oder Verlängerung der Spielwelt sich darbietet, mag die Grundidee der mythopoetischen Welt - Geschichten bringen neue Geschichten hervor, wie auch jede Geschichte in sich potenziell unbegrenzt viele weitere Geschichten birgt - als Spielerfahrung greifbar machen. Außerdem: Ist denn wirklich gewiss, dass der Vulkan unerreichbar bleiben muss? Schließlich erlaubt es uns die SKYRIM-Erweiterung DRAGONBORN (Bethesda Game Studios, 2012), mit der (eigentlich Morrowind zugehörigen) Insel Solstheim einen außerhalb der Weltkarte des Hauptspiels gelegenen Ort zu bereisen. Und wer kann schon mit Sicherheit sagen, welche Geheimnisse sich in einer Welt verbergen, die allein 186 (gestalterisch zugegebenermaßen repetitive) Dungeons bereithält?

Und dann gibt es in SKYRIM ja noch eine schwindelerregende Zahl von Büchern zu entdecken: politische, historische oder militärische Abhandlungen, philosophische, alchimistische und religiöse Traktate, Lyrikbände, Sagen, Märchen, Erzählungen ... hunderte von Seiten, die die Spielerinnen und Spieler, so

118 Franziska Ascher: Immersion. Die Faszinationskraft virtueller Welten. In: Paidia. Zeitschrift für Computerspielforschung, 27. August 2012, http://www.paidia.de/immersion/ [letzter Zugriff: 12.05.2020]. 
sie genügend Muße mitbringen, allesamt lesen können. Nicht nur, dass diese Bücher beinah überall herumliegen (was immer man sonst über Skyrims Bauern, Händler, Gastwirte, Soldaten, Stadtwachen, Banditen, Schwarzmagier oder Vampire denken mag: Anscheinend haben wir es mit fleißigen Lesern zu tun), sie enthalten tatsächlich eine Menge Wissenswertes über die Spielwelt. Zum einen tragen die Bücher also dazu bei, wie Anton Dietrich sagt, „eine lebendige und glaubwürdige Welt zu schaffen, an deren Mehrdimensionalität und Tiefe nur wenige Spiele herankommen“. ${ }^{119}$ Meines Erachtens erfüllen die Bücher in SKYRIM allerdings noch eine weitere wichtige Funktion, insofern sie die Spielwelt dabei unterstützen, die eigenen Grenzen zu verwischen. Diese zweite Funktion zielt darauf, ein Gefühl der Unklarheit $\mathrm{zu}$ erzeugen, was unsere Möglichkeiten betrifft, die (topografischen, geschichtlichen, metaphysischen) Tiefen der Welt von SKYRIM auszuloten. Beispielsweise kann man eine Reihe von Werken aus der Feder des Gelehrten Thelwe Ghelein finden, die sich mit einem geheimnisvollen, technologisch überaus avancierten, „Dwemer“ genannten Zwergenvolk befassen, das längst schon aus Skyrim verschwunden ist. ${ }^{120}$ In Dwemer Inquiries Vol III liest man die Sätze:

I have found in a small number of ruins reference to a geological anomaly or place known as „Fal'Zhardum Din“. This is intriguing because the term not only appears in a few tablet fragments, but very specifically on ornate metal frames in the deepest reaches

119 Anton Dietrich: Welten schaffen. Das Buch als Konstruktionselement des Computerspiels Teil 1. In: Paidia. Zeitschrift für Computerspielforschung, 31. Mai 2017, http://www.paidia.de/ welten-schaffen-das-buch-als-konstruktionselement-des-computerspiels-teil-1/ [letzter Zugriff: 12.05.2020].

120 In der Konstruktion des Dwemer-Reiches verbindet SKYRIM mittelalterliche und viktorianische Phantasmen. Kevin und Brent Moberly erläutern: „While this sort of bricolage typifies the way that the ELDER SCROLLS franchise differentiates races and cultures, the suggestion here is that the Dwemer represent something else altogether: a high-tech, steam-driven strand of medievalism, one that in its conspicuous aesthetic primitivism constitutes as much of an origin myth as the more traditional, heroic, and anti-technological strands of medievalism that players encounter ingame. Understood in this sense, it is perhaps best to describe the Dwemer aesthetic as steampunk.“ Kevin Moberly/Brent Moberly: Swords, Scorcery, and Steam. The Industrial Dark Ages in Contemporary Medievalism. In: Karl Fugelso/Vincent Ferré (Hg.): Medievalism on the Margins. Cambridge 2015, S. 193-216, hier: S. 200. In ihrer politischen Interpretation der so entstehenden ästhetischen Zeitlichkeit kommen die beiden zu dem Schluss: „This ideology, in other words, interpellates us as medieval subjects - the backward remnants of a passing order whose inability to look beyond the traumas and inequities of the present are symptomatic of a larger failure to embrace the promise of a digital renaissance that is always only a microprocessor away. Medievalism, as such, is ideally suited to interrogate the way that this and any number of other fantastic futures are sold to the public through the expedient of reimagining the present as a version of the medieval past.“ Moberly/Moberly: Sword, Scorcery, and Steam, S. 215. 
of the Strongholds Alftand, Irkgnthand [sic] and Mzinchaleft of Skryim. I have yet to decipher the meaning of these elaborate carvings, but consider it highly strange that they occur in the deepest part of each of these ruins. ${ }^{121}$

Und weiter heißt es: „The most reasonable translation of ,Fal'Zhardum Din“ I have managed to decipher is ,Blackest Kingdom Reaches‘, but I cannot imagine what that means.“122 Wer auf Gheleins Bücher stößt, wird sich gewiss fragen, was es mit diesen rätselhaften „Blackest Kingdom Reaches“, meist kurz „Blackreach“ genannt, auf sich haben mag. Wahrscheinlich werden viele Spielerinnen und Spieler, die Thelwe Gheleins Schriften durchblättern, auch den Wunsch verspüren, selbst in die mysteriösen Tiefen des Dwemer-Reiches vorzustoßen - ohne freilich aus dem Text selbst erraten zu können, ob die Entwickler hier einfach Hintergrundinformationen zur Verfügung stellen, die der Welt von SKYRIM eben „Mehrdimensionalität und Tiefe“ verleihen, oder ob die Forschung von Thelwe Ghelein in irgendeiner Form für das erlebbare, gestaltbare, spielbare Spiel von Relevanz ist.

Tatsächlich wird man spätestens im Rahmen der zur Hauptgeschichte zählenden Queste „Elder Knowledge“ ins „Blackreach“ vorstoßen. Und hier nicht einfach einen weiteren, mehr oder weniger beliebigen Dungeon erkunden - sondern eine gewaltige, unterirdische Höhlenstadt, die von riesigen, leuchtenden Pilzen in ein bläuliches Licht getaucht wird, dabei zugleich andersweltliche Schönheit und eine Stimmung schwermütiger Verlorenheit sowie allgegenwärtiger Bedrohung ausstrahlt. Die horrible Anmutung des Ortes ist nicht zuletzt dem Umstand geschuldet, dass hier die Falmer hausen: Ehemals Schneeelfen, wurden sie von den Dwemer versklavt und grausam misshandelt, sodass sie nun als blutgierige, hasserfüllte und - infolge des jahrhundertelangen unterirdischen Daseins - erblindete Monstren durch die Schwärze streifen, mithin wohl nicht zufällig an den „Little People“-Mythos gemahnen, der Autoren wie Arthur Machen, H. P. Lovecraft und Robert E. Howard $\mathrm{zu}$ bedeutenden Werken inspiriert hat und noch in zeitgenössischen Horrorfilmen, wie etwa THE DESCENT (Neil Marshall, GB 2005), ABSENTIA (Mike Flanagan, USA 2011) oder THE HALLOw (Corin Hardy, GB/USA/IRL 2015), ein Echo findet. ${ }^{123}$

121 Zitiert nach: The ELDER SCROLLS-Wiki, https://elderscrolls.fandom.com/wiki/Dwemer_Inqui ries_Vol_III_(Skyrim).

122 Zitiert nach: The ELDER SCROLLS-Wiki, https://elderscrolls.fandom.com/wiki/Dwemer_Inqui ries_Vol_III_(Skyrim).

$123 \mathrm{Vgl}$. zu den ideologischen Grundlagen und dem literaturhistorischen Einfluss des „Little People“-Mythos: Jeffrey H. Shanks: Evolutionary Otherness. Anthropological Anxiety in Robert E. Howards „Worms of the Earth“. In: ders./Justin Everett (Hg.): The Unique Legacy of Weird 
Jedenfalls kann man davon ausgehen, dass ein Spieler, der zuerst in einem Buch vom „Blackreach“ gelesen und sehr viel später dann den Ort selbst entdeckt hat (oder umgekehrt: vermittels der Lektüre herausfand, dass die unterirdische Stadt, in welche er auf der Suche nach einer „Elder Scroll“ gelangte, in Skyrim zu den großen Rätseln der Wissenschaft zählt), die Spielwelt fortan als Ort erleben wird, dessen topografische Ausdehnung um ein Vielfaches überschritten wird von seiner erfahrungsästhetischen Weite. Hier kommt die Strategie einer poetologischen Extension der Spielwelt zum Einsatz, deren Zielpunkt tatsächlich jenseits des Horizonts liegt - die die Möglichkeit einer endlosen Abenteuerstraße, wie Tolkien sie besingt, indessen in der Konstruktion einer „Abenteuerzeit“ momentweise glaubhaft macht.

Doch nicht nur Ausblicke über den Rand der Weltkarte und die Auslotung ungeahnter Erkundungspotenziale im Zusammenspiel von innerdiegetischer Lektüre und dem Eintritt in verborgene - sozusagen apokryphe - Räume sind in diesem Zusammenhang von Bedeutung; auch die Musik spielt eine wichtige Rolle. ${ }^{124}$ Darum will ich mir erlauben, meine analytische Skizze mit einigen

Tales. The Evolution of Modern Fantasy and Horror. Lanham/Boulder/New York/London 2015, S. 119-130, v. a. S. 121-123.

124 Und nicht sie allein. Meine Ausführungen beziehen sich ja ausschließlich auf das, um im Jargon zu sprechen, Vanilla-SKYRIM. Der anhaltende Erfolg des Spiels verdankt sich aber maßgeblich einer überaus eifrigen Community, die vor allem die PC-Fassung von SKYRIM fortwährend mit einer Unzahl an kleinen, größeren und riesigen Mods versorgt und dabei zum Teil auf allerhöchstem Niveau arbeitet. Der Gedanke scheint mir naheliegend, dass die Modder die Verheißung der mythopoetischen Welt in immer neuen Abänderungen des Programmcodes gleichsam auf Materialebene zu verwirklichen suchen. Sun-ha Hong erklärt: „This role of the player is most explicit in their production of paratexts. In its original context of literary interpretation, paratext denotes supplementary material that accompanies the main text of a published book, including front matter, cover art, and so forth. Games accrue an even wider variety of paratextual material, some of it user created. For instance, modding involves an external manipulation of the game's code and other technical assets by the players. Some mods apply specific changes, such as adjusting the color of the sky in SKYRIM. Others rework the [...] [game] wholesale, perhaps transforming SKYRIM’s ,Nordic ' tundras into swampland or presenting an entirely different plot." Und er fährt fort zu erläutern, dass die Modder mitunter so weit gehen, die Art und Weise, wie sich SKYRIM eine phantasmatische Vergangenheit in seinem Weltentwurf aneignet, neu zu interpretieren: „The very extensional nature of this work means that it is not reducible to the standards of historical accuracy alone or even to the authorial intentions of the game developers. Rather, it reflects the players' acquired understanding of and taste for the ,real enough' these games provide. A ,realism' mod thus aspires not to factual accuracy over all other concerns but to factual accuracy in order to more effectively tap into the grand tapestry of heroism - a history defined not by ,what actually happened" but a place and time where ,things of note happen“." Sun-ha 
möglicherweise idiosynkratischen, ganz sicher aber spekulativen Überlegungen zum Score von SKYRIM zu beschließen. Spekulativ sind die folgenden Gedanken allein schon deshalb, weil mir die musikwissenschaftliche Fachkompetenz fehlt. Fest steht, dass sich Jeremy Soules Kompositionen zu der ELDER-SCROLLS-Reihe, er zeichnet auch für den Score von MORROWIND und OBLIVION verantwortlich, größter Beliebtheit erfreuen. Zahlreiche YouTube-Videos, die Musik aus den Spielen in allerlei Kompilationen und Bearbeitungen verfügbar machen und es zusammengenommen auf zig Millionen Klicks bringen, zeugen davon. Zweifelsohne versteht Soule sein Handwerk. Das allein ist aber keine sonderlich befriedigende Erklärung für den Erfolg seiner Musik. Mir scheint vielmehr, dass vor allem der Score von SKYRIM - und insbesondere vielleicht das Stück „From Past to Present“ - das (idealiter sich einstellende) Spielgefühl gleichsam als Klangerfahrung verwirklicht; oder vielleicht gar dafür Sorge trägt, dass ein solches Spielgefühl überhaupt als distinkte Erfahrungsqualität hervortreten kann.

Wie ist das zu verstehen?

Melanie Fritsch benennt „eine ganze Reihe von Ansprüchen“, welchen die Musik im Videospiel zu genügen habe; im Einzelnen soll sie

1. als sinnvolles musikalisches Ganzes funktionieren und insgesamt überzeugend und zufriedenstellend klingen,

2. an dramaturgisch wichtigen Punkten unterstützend wirken,

3. dem Spieler akustisches Feedback auf seine Handlungen und die Spielereignisse geben,

4. nicht repetitiv sein und bei jedem Spieldurchgang ein neues Klangerlebnis bieten,

5. nicht zu viel Speicherplatz benötigen,

6. technisch und musikalisch sinnvolle und flüssige Übergänge bieten,

7. das Setting beziehungsweise die Grundstimmung des Spiels berücksichtigen,

8. mit vertretbarem Kostenaufwand produzierbar sein. ${ }^{125}$

Das Phänomen, das ich zu erfassen suche, verbindet sich wohl am ehesten mit den Punkten zwei, drei und sieben dieser Liste. Aber die Erklärungsansätze, die mit derartigen „Ansprüchen“ einhergehen, überzeugen im gegebenen Fall nur sehr eingeschränkt. Viele der beliebtesten Kompositionen, unter anderem auch „From Past to Present“, begleiten die Spielerinnen und Spieler

Hong: When Life Mattered. The Politics of the Real in Video Games' Reappropriation of History, Myth, and Ritual. In: Games and Culture 10 (2015), H. 1, S. 35-56, hier: S. 48.

Um dem Modding von SKYRIM theoretisch und analytisch beizukommen, wäre freilich eine eigene Untersuchung notwendig.

125 Melanie Fritsch: Musik. In: Beil/Hensel/Rauscher (Hg.): Game Studies, S. 87-107, hier: S. 98-99. 
ja bei ihren Erkundungen der nördlichen Landschaften. Das heißt also, Soules elegische Streicher und Bläser erklingen häufig genug, während der Spieler durch einen sonnenbeschienenen Wald spaziert und ein einsamer Fuchs vor ihm über den Pfad schnürt. In solchen Situationen ist die Musik weit davon entfernt, dem Spieler ,akustisches Feedback auf seine Handlungen und die Spielereignisse“ zu geben; ebenso wenig wirkt sie dramaturgisch unterstützend. Im Gegenteil könnte man sagen, dass hier ein eklatantes Missverhältnis zwischen dem Pathos der Komposition, in ihrer Modulation des romantischen Idioms, und dem ziemlich trivialen, jedenfalls unspektakulären Spielgeschehen entsteht. Eigentlich müsste die solcherart sich vollziehende affektive Überladung sehr gewöhnlicher Spielmomente läppisch, vielleicht gar lachhaft wirken. Doch dem ist nicht so. Und das wiederum kann ich mir nur so erklären, dass die Musik Setting und Grundstimmung des Spiels weniger berücksichtigt, als vielmehr erst herstellt, oder doch miterzeugt.

In seiner Studie über audiovisuelle Rhythmik im Medium Film zitiert JanHendrik Bakels eine Passage, in der Theodor W. Adorno und Hanns Eisler die Funktionsweise des Leitmotivs in Wagners Opern zu bestimmen suchen.

Die beiden schreiben:

Das Wagnersche Leitmotiv ist untrennbar verbunden mit der Vorstellung vom symbolischen Wesen des Musikdramas. Das Leitmotiv soll nicht einfach Personen, Emotionen oder Dinge charakterisieren - obwohl es weithin immer so aufgefasst worden ist -, sondern es soll im Sinn der eigentlichen Wagnerschen Konzeption die szenischen Vorgänge in die Sphäre des metaphysischen Bedeutenden erheben. Wenn im Ring das Walhallmotiv in den Tuben ertönt, so soll es nicht Wotans residence anmelden, sondern Wagner wollte damit die Sphäre des Erhabenen, des Weltwillens, des Urprinzips ausdrücken. ${ }^{126}$

Freilich ist es Adorno und Eisler hier um eine Kritik an Filmkomponisten zu tun, die bei ihren Arbeiten die Leitmotiv-Technik in der Tradition Wagners verwenden. Wie Bakels ausführt, zielt diese Kritik auf den „Vorwurf der schlichten ,Verdopplung“ visueller Repräsentationen“, da „jene höhere Symbolik“ nach der Wagner strebte, „dem Film qua seiner medialen Spezifik als vermeintlich realitätsabbildendes Medium verstellt" bliebe. ${ }^{127}$

Die Betonung liegt wohlgemerkt auf vermeintlich; denn Bakels weist die Kritik von Adorno und Eisler ebenso zurück wie die Vorstellung, dass der Film als repräsentationales Medium zu verstehen sei. Im Gegenzug entwickelt

126 Theodor W. Adorno/Hanns Eisler: Kompositionen für den Film. Frankfurt a. M. 2006, S. 13. Vgl.: Jan-Hendrik Bakels: Audiovisuelle Rhythmen. Filmmusik, Bewegungskomposition und die dynamische Affizierung des Zuschauers. Berlin/Boston 2017, S. 15.

127 Bakels: Audiovisuelle Rhythmen, S. 15. 
er folgende These zum Leitmotiv im Film: „Das musikalische Leitmotiv bildet ein kompositorisches Muster aus, welches innerhalb der linearzeitlichen Erfahrung des Films zeitlich nicht in einem Kontinuum verbundene Szenen über ein sinnlich-körperlich erfahrenes Moment der Gemeinsamkeit verbindet.“128 Und weiter heißt es: „Das Leitmotiv transzendiert als expressive Form Szenen unterschiedlicher Gefühlsqualität - und wird so zu einer sinnlich-körperlich erfahrenen Verbindung, die unterschiedliche audiovisuelle Kompositionen aufeinander bezieht, moduliert und vertieft." 129 Eine von Bakels' Schlussfolgerungen lautet, dass die „nicht-sprachliche, mythische Qualität des Leitmotivs vor diesem Hintergrund auch abseits jedweder Symbolik als eine Gefühlsqualität denkbar“ sei. ${ }^{130}$ Allgemeiner formuliert: „Über das Leitmotiv eröffnet sich eine affektdramaturgische, d.h. die Entfaltung von Zuschauergefühlen ästhetisch grundierende, Struktur.“131

Nun weiß ich nicht, inwieweit Jeremy Soules Arbeit von Wagner beeinflusst ist; Leitmotive verwendet er jedenfalls oft und gerne. So will ich nun den Versuch unternehmen, Bakels' Thesen kurzerhand auf das Videospiel zu übertragen und die Vermutung äußern, dass Soules Kompositionen dem „Plötzlich!“ und dem „Gerade!“ der „Abenteurerzeit“ eine mythische Gefühlsqualität gegenüberstellen, beziehungsweise jene mit dieser zusammenschließen. Es mag sein, dass solcherart „eine affektdramaturgische, d. h. die Entfaltung von Zuschauergefühlen ästhetisch grundierende, Struktur" entsteht, die idyllische Waldspaziergänge mit epischen Schlachten und Explorationen am Rande der Wirklichkeit verbindet; und all das einfasst in die melancholische Poesie einer zauberischen Welt, die gleichwohl von Bürgerkrieg, Rassismus und der Wiederkehr eines uralten Bösen, des seelenverschlingenden Drachen Aluin, verheert ist. Sodass Soules Musik wesentlich dazu beiträgt, dass in der Tat eine Sehnsucht nach dem ganz Anderen als „Grundgefühl“, oder grundlegendes Spielgefühl, von SKYRIM entsteht; vielleicht auch und gerade dann, wenn gar nichts Besonderes geschieht in der Spielwelt, denn „zeitlich synchrone Wahrnehmungen von Bild und Ton“ können - wie Bakels unter Bezug auf Michel Chions Konzept der „Synchrese“ feststellt $^{132}$ - ,in einer gemeinsamen Wahrnehmungsqualität, die über die Qualitäten der einzelnen modalen Eindrücke hinausgeht, aufgehen“. ${ }^{133}$

128 Bakels: Audiovisuelle Rhythmen, S. 17-18.

129 Bakels: Audiovisuelle Rhythmen, S. 18.

130 Bakels: Audiovisuelle Rhythmen, S. 19.

131 Bakels: Audiovisuelle Rhythmen, S. 19.

132 Vgl. Michel Chion: Audio-Vision. Sound on Screen. New York 1994, S. 63-65.

133 Bakels: Audiovisuelle Rhythmen, S. 36. 
Schon in einem letztlich recht sorglosen Fantasy-Blockbuster wie SKYRIM ${ }^{134}$ verbindet die Sehnsucht nach dem ganz Anderen die Spielerinnen und Spieler mit einer großen Zahl an Spielfiguren: als die, nur zu oft vergebliche, Hoffnung, dass die Welt in ihrer Immanenz sich selbst transzendieren möge. THE BANNER SAGA nimmt dieser gespielten und erspielten Sehnsucht, wie wir sogleich sehen werden, viel von ihrem Spielerischen; hier wird sie zu einem Gefühl existenzieller und mitunter verzweifelter Not, das die Spielwelt ebenso wie das Spielgefühl ganz durchwirkt.

134 Die ELDER-SCROLLS-Reihe, und vielleicht insbesondere OBLIVION und SKYRIM, sind durch eine innere Konsequenzlosigkeit gekennzeichnet, die die Spiele recht herausfordernd macht, wenn es um das Konzept von Gut und Böse als metaphysischem Baustoff und die Eukatastrophe geht. In SKYRIM etwa ist für den Avatar eine, wie Ascher sagen würde, „Heldenkarriere“ vorgesehen, die ihn in gerader Linie zum Weltenretter macht. Zugleich jedoch darf er ein reißender Werwolf (oder ein blutgieriger Vampir), der Anführer der Diebesgilde und der Oberassassine der Dark Brotherhood sein - alles auf einmal, alles zugleich.

Anhand der Questen der Mörderbruderschaft ist Markus Engelns der Frage nachgegangen, wie der Held in SKYRIM zum Schurken werden kann, und was das für die Moralität des Spiels bedeutet. Dabei greift er - eine Parallele zu Baumgartners Ansatz - auf Gernot Böhmes Atmosphärebegriff zurück, um sich einen analytischen Zugang zu erschließen. Engelns Conclusio liest sich wie folgt: „Anders als in den anderen Gilden, inszeniert das Spiel aber eine gewichtige und allseits vorhandene ,Atmosphäre der Entlastung', bei der nicht jede moralisch fragwürdige Handlung einzig auf den Spieler allein zurückgespiegelt werden kann. Insbesondere die Anwendung von Böhmes Kategorien zeigt doch in der gesamten Analyse, dass der Spieler ein Stück weit von seiner eigenen Spielfreiheit entkoppelt ist und sich in einem Machtgefüge wiederfindet, durch das er sich wie vom Spiel gewünscht verhält; entweder er vernichtet die Dunkle Bruderschaft, was das Spiel explizit vorsieht und entlohnt, oder er folgt ihr und begibt sich damit in die archaische Nebenwelt, die von Gruppenzugehörigkeiten und dem Druck von ,oben' geprägt ist. Der atmosphärische Aufwand, den das Spiel dazu betreibt, lässt allerdings hinsichtlich der gesellschaftlichen Problematik des Akts des Mordens in Computerspielen aufatmen - immerhin müsste das Spiel keinen solchen Aufwand betreiben, wenn es Mord zweifelsfrei und ohne jeden gesellschaftlichen Diskurs als probate Problemlösungsstrategie anerkennen würde. Der eigentliche Reiz der Gildenquest besteht eben darin, die verschiedenen Ebenen überhaupt erst diskursfähig zu machen und das durch die Kollision von Spielprozess, Atmosphäre und Moralvorstellungen entstandene Spannungspotenzial auszunutzen, um daraus eine besondere Spielerfahrung zu realisieren. "Markus Engelns: Vom Helden zum Mörder?, S. 150-151.

Ich bin mir unsicher, ob es angeht, die Spielerinnen und Spieler, beziehungsweise den Weltentwurf von SKYRIM, solcherart zu „entlasten“. Vielleicht verhält es sich so, dass die Spielwelt nur dann „gut“ sein, also in Entsprechung zu ihren poetologischen Prinzipien funktionieren kann, wenn das Gute und das Böse in ihr mehr oder weniger gleichberechtigt bestehen dürfen. Jedenfalls wäre auch diese Frage eine eigene Untersuchung wert. 


\section{Eine Welt nach ihrem Ende: THE BANNER SAGA}

Von Anfang an wird deutlich, dass THE BANNER SAGA ernst macht mit dem Pathos der Fantasy. ${ }^{135}$ „The gods are dead“ - dies ist er erste Satz, den die Spielerinnen und Spieler zu lesen bekommen. Er erscheint auf einer verwitterten, halb verdüsterten und mit stilisierten Schriftzeichen bedeckten Karte. Dort stehen Namen wie „Hraun“, „Frostvellr“ oder „Grofheim“, die nordisch oder altnordisch anmuten, wozu sicherlich auch beiträgt, dass die Schriftzeichen, in denen sie gehalten sind, an germanische Runen erinnern - was wiederum den Eindruck der Fremdheit verstärkt, da einige der Ortsbezeichnungen auf den ersten Blick kaum zu entziffern sind.

THE BANNER SAGA belässt es nicht bei dem ebenso nüchternen wie rätselhaften - und je nach Perspektive der Spielerinnen und Spieler durchaus grausigen - Hinweis, dass die Götter gestorben seien. „In their wake, man and giant survived through a tenuous alliance, driving black destroyers called dredge deep into the northern wastes“, so der zweite Satz, den wir zu lesen bekommen. Vom unteren Bildschirmrand her schieben sich die Buchstaben in unser Sichtfeld, als müsste da etwas aus großer Tiefe herbeigeschafft werden: Die Zeitenferne einer anderen Epoche öffnet sich, die Schatten historischen Vergessens rücken näher. Als nächstes heißt es: „Now is an era of growth and trade. Life goes on.“ Schließlich der letzte Satz: „Only one thing has stopped. The sun.“

Was für eine kühne Exposition! Kühn ist sie nicht vorwiegend deshalb, weil sie sehr viel mehr Fragen aufwirft, als sie beantwortet; dergleichen sind genreaffine Spielerinnen und Spieler gewohnt vom Einstieg in Fantasy-Geschichten. Ihre Radikalität rührt vielmehr daher, dass hier in wenigen Worten eine Welt vorgestellt wird, die zwischen Katastrophe und Eukatastrophe, verstanden als geschichtsbildende Prinzipien, zerrissen und mit sich selbst aufs Äußerste zerfallen ist. Die Götter sind tot, aber ein Zeitalter des Wachstums und des Handels ist heraufgezogen; die Menschen und Riesen haben die Dredge zurückgeschlagen - in einem Krieg, dessen Grausamkeit durch das Bild der schwarzen Zerstörer evoziert wird -, aber ihre Allianz scheint brüchig; das Leben geht weiter, aber die Sonne steht still.

135 Eine Variante dieser Analyse, die sich auf die kapitalismuskritischen Thesen von JeanPierre Le Goff bezieht, ist erschienen unter: Daniel Illger: Der ohnmächtige Held. Unmögliche Entscheidungen in THE BANNER SAGA. In: Martin Hennig/Marcel Schellong (Hg.): Überwachung und Kontrolle im Computerspiel. Glückstadt 2020, S. 75-104. Vgl. auch Jean-Pierre Le Goff: La barbarie douce. La modernization aveugle des enterprises et de l'école [1999]. Paris 2003. 
Wir haben es also mit einer buchstäblich post-apokalyptischen Welt zu tun. Einer Welt, die ihr eigenes Ende überlebt hat. Einer Welt, in der die Sonne selbst aufgegeben zu haben scheint. Auf der einen Seite metaphysische Trauer; auf der anderen Seite der Hunger nach dem schieren Dasein. Es ist der ebenso hoffnungslose wie unbedingte Wunsch, dass es weitergehen möge. Irgendwie. Wohin und wozu auch immer.

Das ist, kurz gesagt, das Weltgefühl, welches THE BANNER SAGA erzeugt. ${ }^{136}$ Es bestimmt das Spiel, durchdringt das Erleben der Spielerinnen und Spieler. Die Entwickler der Stoic Studios haben die grundlegenden Gesetzmäßigkeiten ihres Weltentwurfs dabei auf konkretistische Weise in Spielmechaniken und -abläufe übertragen: „Life goes on“ - diese Feststellung oder Forderung herrscht, gleichsam als unerbittlicher erfahrungsästhetischer Imperativ, über einem Spielprinzip, welches stets aufs Neue in der Erkenntnis sich aktualisiert, dass es, im Wortsinn, immer weiter gehen muss; wobei diejenigen, die immer weiter gehen, lange Züge von Kriegern und Flüchtlingen, Menschen und Riesen sind, deren Marsch zwar Ziel-, jedoch keine Endpunkte kennt. Die Sonne steht still - der Stasis des erstarrten, gefrorenen Winterlichts entspricht ein Spielverlauf, der, dem unablässigen Vorwärtsdrang des Marsches zum Trotz, bis zur Bewegungslosigkeit in zyklischer Repetition sich ergeht, getaktet durch die Notwendigkeiten des Gehens (der Marsch der Karawanen, die der Spieler verwaltet), des Redens (die unzähligen, folgenschweren Gespräche, die er führen muss) und des Kämpfens (vor allem gegen die Dredge, aber auch gegen vermeintliche Verbündete; solche die auf derselben Seite stehen sollten oder hätten stehen können). ${ }^{137}$ Schließlich gibt es die Drohung der „black destroyers“ aus den Einöden des höchsten Nordens ${ }^{138}$ - die existenzielle Unentrinnbarkeit dieser Drohung schlägt sich darin nieder, dass man in einem fort gezwungen ist, Entscheidungen zu treffen; mitunter sind dies sehr schwere

136 Meine Anmerkungen beziehen sich vorwiegend auf den ersten Teil der Trilogie; im Wesentlichen gelten sie aber gleichfalls für THE BANNER SAGA 2 (Stoic Studio, 2016) und THE BANNER SAGA 3 (Stoic Studio, 2018); die Spiele erzählen eine fortlaufende Geschichte, sind im selben Grafikstil gehalten, und auch das Fundament der Spielmechaniken bleibt sich gleich.

137 Im zweiten und dritten Teil der BANNER SAGA ist der Spielverlauf insofern weniger einförmig, als die Kämpfe selbst abwechslungsreicher gestaltet sind und das Geschehen öfter als zuvor von kleinen filmischen Einlagen oder dramaturgisch fixierten und ausgestalteten Sequenzen aufgelockert wird. Allerdings bin ich mir nicht sicher, ob die Intensität des Spielgefühls, in einer verfallenden Welt ums nackte Überleben zu kämpfen, nicht ein Stück weit der Gleichförmigkeit des Trilogie-Auftakts geschuldet (beziehungsweise zu verdanken) sein könnte.

138 Und als ob das nicht schon schlimm genug wäre, kommen im Verlauf der Trilogie noch zwei weitere Drohungen hinzu: Sie haben die Gestalt einer weltenverschlingenden Riesenschlange und einer metapysischen Finsternis, die unaufhaltsam näherrückt. 
und bittere Entscheidungen, sodass sich THE BANNER SAGA, wenn man Pech hat, bald darstellt als Folge von Abschieden und Totenklagen. ${ }^{139}$

Es ist vor allem dieser letzte Aspekt, der sowohl Spielverlauf als auch Spielerfahrung strukturiert. THE BANNER SAGA besteht aus nichts anderem als Entscheidungen. In gewisser Weise gilt das natürlich für jedes Spiel. Welchen Gegner attackiert man zuerst? Attackiert man überhaupt, oder sucht man lieber das Weite? Geht man die Treppe hinauf oder die Treppe hinunter? Erledigt man zuerst diese oder jene Mission? In den meisten Spielen sind unablässig derartige Entscheidungen zu treffen - und natürlich nicht nur, wenn es um Fantasy geht.

Sebastian Domsch erläutert:

No other medium provides its users as consistently with nodal situations that involve choice as do video games. All video games are rule-bound systems, and these rules constantly define the range of options that a player has in a specific situation (that is: whether the player has a choice or not, and which choice or choices) as well as the consequences of actualizing each of these options. Choice is what video games are all about, even though the reach of agency is not always as extensive as it might be perceived by the player. ${ }^{140}$

139 In der Art, wie THE BANNER SAGA einerseits die Notwendigkeit, Entscheidungen zu treffen, in den Mittelpunkt der Spielerfahrung stellt, es den Spielern und Spielerinnen andererseits nahezu unmöglich macht, zufriedenstellende Entscheidungen zu treffen, kann das Spiel von Stoic Studio durchaus als beispielhaft für das gelten, was die Paidia-Redaktion als „Decision Turn“ bezeichnet. Franziska Ascher, Mireya Schlegel und Tobias Unterhuber führen aus: „In guter kulturwissenschaftlicher Tradition entschieden wir uns für die Bezeichnung dieser zu beobachtenden Entwicklung als Decision Turn - ein Begriff, der in aller gebotenen Kürze fassen soll, dass sich eine Art von Computerspielen herauskristallisiert hat, die erstens Entscheidungen in den Vordergrund stellt und zweitens bisher übliche Lösungsstrategien, die Problemstellungen mit einer optimalen Lösung favorisierten, unterminiert. [...] Und selbst dann noch [in Kenntnis aller Konsequenzen, DI] kann die beste Entscheidung einen bitteren Beigeschmack haben oder die Erkenntnis beinhalten, dass die verfügbaren Optionen nicht mehr der Dichotomie gut oder böse entsprechen.“ Franziska Ascher/Mireya Schlegel/Tobias Unterhuber: Vorwort. Der Prinz oder der Frosch? In: Redaktion Paidia (Hg.): „I’ll remember this“. Funktion, Inszenierung und Wandel von Entscheidung im Computerspiel. Glückstadt 2016, S. 9-14, hier: S. 10.

Vgl. zu der Frage, inwieweit Videospiele mit „katastrophischem“ oder „(post-)apokalyptischem Setting“ besonders dafür geeignet sind, die Spielerinnen und Spieler mit harten und hoffnungslosen Entscheidungen zu konfrontieren: Maria Kutscherow: Moral in der Apokalypse? Entscheidungen und Entscheidungssysteme in katastrophischen und (post-)apokalyptischen Computerspielen. In: Redaktion Paidia (Hg.): „I'll remember this“, S. 215-233, v. a. S. 221 u. 229.

140 Sebastian Domsch: Storyplaying. Agency and Narrative in Video Games. Berlin/Boston 2013, S. 112. Ich beziehe mich im Weiteren vorwiegend auf Domsch' Klassifikation von Entscheidungen im Videospiel, da sie mir - auch aufgrund einer gewissen Einfachheit und Ein- 
Man hat im Videospiel also die Wahl; ein Videospiel spielen heißt: zwischen verschiedenen Optionen auswählen. Und natürlich geht eine Wahl stets mit einer Entscheidung einher. Wenn ich die Wahl treffe, einen riesigen, feuerspeienden Drachen anzugreifen, entscheide ich mich dafür, das Leben meiner Spielfigur im Kampf gegen dieses Ungetüm zu riskieren (unabhängig davon, wie bedeutsam der Verlust des Figurenlebens im jeweiligen Spiel sein mag). Laufe ich vor dem Drachen davon, treffe ich hingegen die Entscheidung, einstweilen auf die Vorteile zu verzichten, die mir ein Sieg über das Monster einbringen könnte (Erfahrungspunkte, bessere Ausrüstung, einen Sack Gold, die Bewunderung und Unterstützung der Bewohner des nahen Dorfes).

Aber Entscheidung ist ja nicht gleich Entscheidung; und Entscheidungen können auf sehr verschiedene Weisen in ein Spiel implementiert sein. „Choice situations differ in the amount of information that is given about the consequences of the different options“", schreibt Domsch. ${ }^{141}$

Und er führt aus:

A choice situation can contain

- no information: the agent has no reasonable knowledge about anything that might result as a consequence of the options

- incomplete information: the agent is provided with some knowledge about possible outcomes, but no certainty in relation to the probability of the outcomes, and/or the completeness of information about outcomes

- complete information: the agent is provided with certain information about all consequences of all options. ${ }^{142}$

Nach Domschs Dafürhalten sorgen am ehesten die „choice situations“ des zweiten Typus für ein erfüllendes Spielerlebnis. Denn wenn die Spielerinnen und Spieler über „incomplete information“ verfügen, heißt das, „that there are con-

gängigkeit - erlaubt, recht umstandslos zu zeigen, worauf es mir bei THE BANNER SAGA ankommt. Das soll allerdings nicht heißen, dass mit Domsch' Thesen sozusagen das letztgültige Wort der Game Studies bezüglich dieses Gegenstands gesprochen wäre. Beispielsweise legen Martin Hennig und Marcel Schellong differenzierte semiologische Theorien zur Funktionsweise von Entscheidungen im Videospiel vor. Vgl. Martin Hennig: „This game series adapts to the choices you make.“ Eine raumsemantische Typologie von Entscheidungssituationen und die Funktionen seriellen Erzählens in aktuellen Episodenspielen. In: Redaktion Paidia (Hg.): „I'll remember this“, S. 145-165. Vgl. auch Marcel Schellong: „Sorry, but you're in my story now“. Zankende Entscheidende und rivalisierende Entscheidungsdispositive in THE STANLEY PARABLE. In: Redaktion Paidia (Hg.): „'lll remember this“, S. 311-334.

141 Domsch: Storyplaying, S. 114.

142 Domsch: Storyplaying, S. 114. 
flicting arguments for and against each choice that might have probabilities, but no certainties attached to them.“ Und weiter: „This is experienced as a meaningful choice, where the player either has to act according to probability (uncertainty) or has to hierarchise incompatibles." ${ }^{143}$

Wie nun verhält es sich in THE BANNER SAGA? Versuchen wir, uns an einem Beispiel zu vergegenwärtigen, welcher Art die Entscheidungen sind, mit denen sich die Spielerinnen und Spieler hier konfrontiert sehen. In einem von zwei Handlungssträngen schlüpft man in die Rolle des menschlichen Jägers Rook, der gegen seinen Willen zum Anführer eines Zuges von Flüchtlingen wird, die verzweifelt versuchen, sich vor den neuerlich vorrückenden Dredge in Sicherheit $\mathrm{zu}$ bringen (wobei sich später herausstellt, dass die grausigen Steinmenschen selbst auf der Flucht - und auch gar nicht unbedingt so grausig - sind). Ganz zu Beginn dieses Handlungsstrangs, noch in Rooks Heimatdorf Skogr, kommt es zu einem ebenso dramatischen wie unerwarteten Zwischenfall, der den Jäger vor eine Entscheidung stellt, bei der es um Leben und Tod seiner Tochter Alette geht.

In immersiver Rollenprosa erläutert Jörg Luibl, was hier geschieht:

Was mache ich nur? Da taucht ein Koloss aus dem Schatten auf und droht meine Tochter mit einer Keule zu zermalmen. Ich könnte ihr schnell eine Warnung zurufen, damit sie hoffentlich noch ausweicht. Ich könnte das Ungetüm mit einem Pfeilschuss auf den Kopf ablenken. Oder ich stürze mich mit Gebrüll und gezückter Axt in den Kampf. ${ }^{144}$

Dieser Moment ist von wesentlicher Bedeutung für THE BANNER SAGA. Da er sich bereits am Anfang des zweiten von sieben Kapiteln ereignet, gibt er sozusagen den Ton vor, nach dem fortan die Musik spielt. Anders gesagt: Er bestimmt das affektpoetische Gepräge, konturiert den Möglichkeitsraum, in dem sich die Spielerinnen und Spieler wiederfinden. Und wer die BANNER-SAGA-Trilogie mit der Erwartung beginnt, dass schon irgendwie alles gut gehen werde, sieht sich hier unverhofft eines Besseren (oder Schlimmeren) belehrt. Denn wenn Rook (beziehungsweise der Spieler) die falsche Entscheidung trifft, stirbt zwar nicht Alette selbst, wohl aber der junge Krieger Egil, der offenbar in die Tochter des Jägers verliebt ist. Dieser Tod - und das gilt für alle Tode in

143 Domsch: Storyplaying, S. 115.

144 Jörg Luibl: Test. THE BANNER SAGA. In: www.4players.de, 14. Januar 2014, https://www.4play ers.de/4players.php/dispbericht/PC-CDROM/Test/30117/80232/0/The_Banner_Saga.html [letzter Zugriff: 12.05.2020]. 
dem Spiel - ist unwiderruflich; zumindest lässt er sich nicht innerhalb des Erzählkontinuums revidieren, das THE BANNER SAGA entwickelt. ${ }^{145}$

Man muss sich klarmachen, dass das Spiel den Spielerinnen und Spielern eine Freiheit nimmt: jene nämlich, keine Entscheidung zu treffen. Wie gesagt, das gilt in gewisser Weise für jedes Spiel. Doch in The Banner Saga sind die Momente der Entscheidung immer als solche markiert und zugleich mit einem existenziellen Gewicht beschwert: Spielend weiß man, dass das eigene Tun unmittelbar spürbare, häufig schmerzliche und unwiderrufliche Konsequenzen haben wird. Zwar verzichtet THE BANNER SAGA darauf, eine Zeitbegrenzung zu verhängen: Wenn sie wollen, können die Spielerinnen und Spieler stundenlang - auch dies in Entsprechung zu der erstarrten Zeitlichkeit einer Welt, die unter einer gelähmten Sonne ausharren muss - vor dem Bildschirm oder dem Fernseher hocken und an ihren Fingernägeln knabbern. Aber früher oder später müssen sie sich entscheiden, wenn sie vorankommen möchten. Mehr noch: Wir dürfen auch nicht irgendeine Entscheidung treffen. Sondern exakt nur die, welche uns THE BANNER SAGA vorgibt. Wiederum gilt: Das trifft vorderhand auf alle Spiele zu. Doch The Banner Saga trägt stets Sorge, die Spielerinnen und Spieler ihre Unfreiheit spüren zu lassen. Man muss Entscheidungen treffen, hat aber keine Entscheidungsfreiheit - das Spieldesign verschleiert diesen Umstand nicht, sondern offenbart ihn vielmehr, legt ihn stets und allerorten bloß. In den Gesprächen ist es beispielsweise so, dass uns, in der Art eines Spielbuchs, Texttafeln gezeigt werden, auf denen eine literarische Beschreibung der jeweiligen Situation zu lesen steht, verbunden mit der Frage, was wir zu tun gedenken. In dem geschilderten Fall gibt es also ausschließlich die von Luibl genannten Möglichkeiten: 1.) Alette warnen; 2.) einen Pfeil auf den Dredge abschießen; 3.) den Dredge mit gezogener Axt angreifen. Wählt man die erste oder die dritte Option, so stirbt Egil.

So verfährt THE BANNER SAGA in allen vergleichbaren Momenten. Luibl benennt eine Reihe von weiteren Entscheidungen, die den Spieler erwarten:

Ziehe ich mich nach der Sichtung von Qualm und einer Übermacht an Feinden lieber zurück oder forsche ich nach? Locke ich Feinde in einen Wald und zünde ihn an oder warte ich ab? Schlichte ich den Streit in einem Dorf oder ergreife ich Partei? Statuiere ich an

145 Freilich ist es in THE BANNER SAGA, wie in den meisten zeitgenössischen Videospielen, möglich, zu einem früheren Spielstand zurückzukehren. Dies jedoch nicht ohne Weiteres, denn: „a well-judged auto-save-only system means that, while reloading a previous save to undo a poor decision is possible, it will usually result in losing several hours of play. "Stace Harman: THE BANNER SAGA review. Trooping the Colors. In: www.eurogamer.net, 9. Februar 2015 [14. Januar 2014], https://www.eurogamer.net/articles/2014-01-14-the-banner-saga-review [letzter Zugriff: 12.05.2020]. 
einem Alkoholiker ein Exempel oder spiele ich die Sache runter? Lass ich einen alten Kauz Gedichte vortragen oder soll er sich zurückhalten? ${ }^{146}$

Und er merkt an: „Manchmal gibt es nur banale, manchmal aber auch fatale Folgen.“" ${ }^{147}$ Also noch einmal: Mit welchem Typus von "choice situation“ haben wir es in THE BANNER SAGA zu tun haben? Vielleicht ist man geneigt, sie kurzerhand der „incomplete information“-Variante zuzuordnen. Es ist ja nicht so, dass das Spiel die wesentlichen Informationen samt und sonders zurückhalten würde. Wir wissen zum Beispiel, dass der Dredge Alette töten wird, wenn niemand ihn daran hindert; wir wissen auch, dass Rook ziemlich gut mit Pfeil und Bogen umgehen kann; wir wissen darüber hinaus, dass sich noch andere Figuren in Alettes Nähe aufhalten: etwa der verrückte Speerkämpfer Tryggvi oder eben Egil; vielleicht ahnen wir sogar, dass Egil versuchen wird, Alette zu beschützen, wenn wir bei dem Versuch, den Dredge zu töten, scheitern sollten.

Allerdings erlaubt uns nichts von all dem, die Konsequenzen der Entscheidung „Warnung“, „Pfeil“ oder „Axt“ abzusehen. Das liegt daran, dass die Informationen, die uns THE BANNER SAGA zur Verfügung stellt, auf einer völlig anderen Ebene angesiedelt sind als jene, die wir benötigen würden, um zu diesem frühen Zeitpunkt, da wir gerade erst begonnen haben, die Spielwelt kennenzulernen - die Wahrscheinlichkeit zu kalkulieren, ob unser Tun fatale Folgen herbeiführen könnte. Letztlich ist die Frage nämlich nicht: Kann Rook gut genug mit dem Bogen schießen? Sondern: Haben wir es mit einem Spiel zu tun, das wichtige Figuren in der Beiläufigkeit eines Klicks sterben lässt? Und vor allem: Bürdet THE BANNER SAGA die Verantwortung für den Tod der Figuren den Spielerinnen und Spielern auf, oder vollziehen sich die Unglücksschläge hier mit schicksalhafter Unausweichlichkeit, sind mithin unserer Einflussnahme entzogen?

Es geht also weniger um die Fülle oder Spärlichkeit der Informationen als um eine Einschätzung der Gesetzmäßigkeiten, denen die Spielwelt unterliegt. Die Prinzipien, nach denen eine Welt gebaut ist, bestimmen gewissermaßen den Radius dessen, was in der entsprechenden Welt geschehen kann; nur wer sie erschlossen hat, verfügt über die Interpretationsmatrix, die es ihm erlaubt, diese oder jene Information richtig zu deuten. Je komplexer und dynamischer das Weltgesetz sich gestaltet, desto schwerer ist es freilich, eindeutige Handlungsanweisungen aus den als solchen erkannten Bauprinzipien abzuleiten. In der geschilderten Szene müssen die Spielerinnen und Spieler erleben, dass Figuren sehr schnell sterben können, wenn sie eine falsche Entscheidung treffen;

146 Luibl: THE BANNER SAGA Test.

147 Luibl: THE BANNER SAGA Test. 
es gibt aber auch Situationen, in denen unverhofft etwas Gutes geschieht, wenn sich beispielsweise herausstellt, dass es möglich ist, ohne Blutvergießen in die Stadt Frostvellr zu gelangen, deren Tore den Flüchtlingen verschlossen sind (was dann freilich, getreu dem Grundprinzip des Spiels, auf verschiedene Weise wieder in eine katastrophische Situation umschlagen kann).

Was man im Fall von THE BANNER SAGA begreifen muss, ist eben, dass die Welt des Spiels - wie bereits ausgeführt - in einem Kampf zwischen Katastrophe und Eukatastrophe begriffen ist, dessen Ausgang darüber bestimmen wird, welche Geschichtlichkeit dieser Welt eignet. Nun ist aber der Ausgang des Kampfes ungewiss; mehr noch: Das Spiel ist in gewisser Weise dieser Kampf. Das heißt also, als affektive Pole kennt die BANNER-SAGA-Trilogie die finsterste, hoffnungsloseste Schwärze ebenso wie das jäh aufflackernde Licht, welches durchs Dunkel schneidet. Allzeit ist beides möglich.

Vor diesem Hintergrund lässt sich Folgendes sagen: Was Domsch „gameplay rationality“ nennt, ist bei THE BANNER SAGA keineswegs gegeben, da die Spielerinnen und Spieler - von den rundentaktischen Kämpfen abgesehen kaum je in der Lage sind, die Konsequenzen ihrer Entscheidungen solcherart abzuschätzen, dass sie das eigene Spiel zuverlässig auf die von ihnen gewünschten Ergebnisse abstimmen könnten. ${ }^{148}$ Was ihnen bleibt, sind mithin „semantic choices“, also Entscheidungen, die sich letztlich stützen auf das Verständnis, oder das mutmaßliche Verständnis, der, wie Domsch sagt, „properties

148 Vgl. Domsch: Storyplaying, S. 124. Auch hierin kann THE BANNER SAGA als beispielhaft für den „Decision Turn“ gelten. Wenn Tobias Unterhuber und Marcel Schellong recht haben, hat sich etwa seit THE WITCHER 2: ASSASSINATION OF KINGS (CD Project Red, 2011) eine deutliche Verschiebung in der Logik ergeben, wie Videospiele ihre Entscheidungssysteme implementieren. Folgt man den Autoren, war es lange Zeit so, dass die Entscheidungen, die die Spielerinnen und Spieler zu treffen hatten, „zwar in ein moralisches Framing eingebettet [waren], dennoch handelte es sich dabei weder um moralische Entscheidungen noch Dilemmata, da andere Überlegungen diese überlagerten: Belohnungen für die,richtige 'Entscheidung oder gar die einzige Möglichkeit des Weiterkommens machten aus den oberflächlich moralischen Entscheidungen eigentlich ökonomische. Damit folgten sie der konventionellen Strategie des Computerspiels, die eben einer ökonomischen Norm der Gewinnmaximierung und Effizienz oder sogar einer neoliberalen Ideologie verpflichtet war.“ Im Zuge des „Decision Turn“ ergab sich u. a. die folgende Veränderung: „Der moralische und handlungsbestimmende Rahmen dieser Entscheidungen veränderte sich merklich. Eine primär ökonomistische Handlungsstrategie allein scheint nicht mehr zielführend zu sein. Dominante Strategien werden insgesamt in ihrer Validität infrage gestellt.“ Tobias Unterhuber/Marcel Schellong: Wovon wir sprechen, wenn wir vom Decision Turn sprechen. In: Redaktion Paidia (Hg.): „I'll remember this“, S. 15-31, hier: S. 18 u. S. 19. 
of the fictional world that the game creates“. ${ }^{149}$ Domsch nennt diese „semantic choices“ auch „narrative choices“ und erläutert:

One example of such narrative choices are all those that are character-based. As has been already discussed, some player choices are also motivated by the player's conception of the character. That is, the player bases the decision on the consideration how well it fits into the pattern of behaviour assumed to be the right one for the character. ${ }^{150}$

Bezogen auf THE BANNER SAGA heißt das: In dem Maße, wie sich die Spielwelt als unkalkulierbar erweist, in dem Maße also, wie sie Gesetzmäßigkeiten enthüllt, die gerade auf Unberechenbarkeit und Unvorhersehbarkeit abzielen, müssen die Spielerinnen und Spieler einen anderen Boden suchen, der fest genug ist, um ihre Entscheidungen zu tragen, als jenen, der mit der Hoffnung auf möglichst erfolgreiches oder effektives Spiel geben ist. ${ }^{151}$ Die Frage ist dann nicht mehr: Welche Handlungsoption bringt mir die größtmöglichen Vorteile ein, oder führt wenigstens keine gravierenden Nachteile herbei? Sondern: Was würde Rook in einer solchen Lage tun? Was würde er für richtig halten? Da Rook sein Leben aber in der Wahrnehmung der Spielerinnen und Spieler findet, in ihrer Einfühlung in die phantasierte Innerlichkeit der Figur, die letztlich ja eine Einfühlung in das Eigene ist - in möglicherweise ungenutzte oder durch die Figur erst greifbar und erlebbar gewordene Seinspotenziale -, geht es nun plötzlich um einen Selbstbezug, der bei einem Spiel wie THE BANNER SAGA durchaus existenzielles Gewicht bekommen kann. Mit einem Mal steht in Frage, welche Idee von Gut und Böse man in der Spielwelt erkennt; und welche Idee von

149 Domsch: Storyplaying, S. 125.

150 Domsch: Storyplaying, S. 125-126. Domsch gibt dazu folgendes Beispiel: „Character-based choices can overlap with and sometimes overrule gameplay rationality. Thus, the tactical choice for a specific weapon can be overruled by the player's idea that ,my character would never use an axe, she is more the bow-and-arrow type', even though choosing the axe might have given a real gameplay advantage.“ Domsch: Storyplaying, S. 126.

151 Britta Neitzel stellt folgende These auf: „Im Gegensatz zur sozialen Interaktion, die unsicher ist, bietet die Interaktivität Sicherheit an. Als Souverän kann sich das spielende Subjekt nicht nur insofern fühlen, als es eine einfache Handlung übersieht und ausführt, sondern auch weil es das System durchschauen kann. Gerade die Möglichkeit, sich im Laufe des Spielens Regel-, Verhaltens- und Interaktionssysteme zu erschließen, unterscheidet Computerspiele von vielen anderen Spielen, in denen ein Regelsystem von vornherein gegeben ist und das Set von möglichen Handlungen vorgibt." Neitzel: Involvement, S. 231. In Hinblick auf das Gros der Spiele, die in der vorliegenden Studie analysiert werden, kommt mir diese Aussage eher zweifelhaft vor; fest steht, dass sich die Spielerinnen und Spieler weder in THE BANNER SAGA noch in SHADOW OF THE COLOSSUS, DARK SOULS oder HELLBLADE - SENUA'S SACRIFICE als „SOuverän“ fühlen können oder sollen. 
Gut und Böse man selbst unter den Bedingungen der Spielwelt zu realisieren bestrebt ist. ${ }^{152}$

Die Vermutung scheint also naheliegend, dass gar nicht unbedingt die Vollständigkeit der Informationen den Ausschlag gibt, wenn die Erfahrungsqualität der Spielerentscheidungen zu bestimmen ist. Damit eine Entscheidung im Sinne von Domsch als „meaningful“ erlebt wird, muss sie vielmehr in ihrer Anlage und ihren Konsequenzen den Gesetzmäßigkeiten jener Welt entsprechen, sodass sich das Weltgesetz gleichsam in der Spielerfahrung erfüllt. Um es etwas schlichter auszudrücken: In einer Welt, wie sie uns THE BANNER SAGA vorstellt, wäre es enttäuschend oder gar empörend, wenn falsche Entscheidungen nicht immer wieder zum Tod wichtiger Figuren führen würden. Denn dann wäre die schmerzliche Bedeutsamkeit, welche das Spiel den Entscheidungssituationen beizugeben sucht, nur eine Vorspiegelung; es läge dann gewissermaßen ein Betrug vor - sowohl am Spieler als auch an der eigenen Poetik.

Da es sich aber nicht so verhält, kann Malindy Hetfeld bezogen THE BANNER SAGA von „the joy of meaningful death“ sprechen. ${ }^{153}$

Sie führt aus:

From a mechanical point of view, it's almost scary how many of your friends you can lose in quick succession. Yet it is important to commit to those deaths, because they are what gives THE BANNER SAGA it's [sic] emotional depth. You're going through war and famine and large-scale destruction, so as frustrating as it may be, there is often no sense to what does or doesn't cause someone's death. It's so impactful because it doesn't always happen in a battle, as a heroic sacrifice. It just does.

[...] It's a great equaliser, in a gaming genre that is so often about having the bigger crew and the better preparation, that you mostly don't know what you're getting into in THE BANNER SAGA. It's a trilogy that told me to not get attached, but of course I did. ${ }^{154}$

152 Auch dies mag bis zu einem gewissen Grad als allgemeines Merkmal von Spielen gelten, die die Entscheidungen der Spielerinnen und Spieler in den Mittelpunkt der Spielerfahrung stellen; allerdings meine ich, dass die Frage nach Gut und Böse in der Fantasy-Interpretation, die THE BANNER SAGA vornimmt, eine durchaus unübliche Dringlichkeit erhält. In diesem Zusammenhang könnte es hilfreich sein, sich mit Julian Reidy darum zu bemühen, Kierkegaards Unterscheidung zwischen „ästhetischen“ und „ethischen“ Entscheidungen aufs Medium Videospiel zu übertragen. Vgl. Julian Reidy: „There are so many choices!“ Zur Entscheidungssituation im Computerspiel. In: Redaktion Paidia (Hg.): „I’ll remember this“, S. 275-290, hier: S. 286.

153 Malindy Hetfeld: The Joy of Meaningful Death in THE BANNER SAGA. In: www.rockpapershot gun.com, 16. Oktober 2018, https://www.rockpapershotgun.com/2018/10/16/the-joy-of-mea ningful-death-in-the-banner-saga/ [letzter Zugriff: 12.05.2020].

154 Hetfeld: The Joy of Meaningful Death in THE BANNER SAGA. 
Nun ergibt sich freilich ein weiteres Problem. Denn wenn es zutreffen sollte, dass die Entscheidungen, die die Spielerinnen und Spieler in THE BANNER SAGA zu treffen haben, darum so „meaningful“ sind, weil sie sich im Einklang mit den grundlegenden Gesetzmäßigkeiten oder Bauprinzipien der Spielwelt vollziehen, muss ja noch geklärt werden, was in diesem Fall überhaupt unter einer „Welt“ $\mathrm{zu}$ verstehen ist.

Bei einem 3D-Open-World-Spiel wie SKYRIM ist das offensichtlich: Man kann die Welt ja durchwandern und durchreiten; sie bietet sich dar in weiten, sonnigen Ebenen, nebelverhangenen Wäldern und Berggipfeln, die von Schnee und Wind umtost werden; und sofern ihnen der Sinn danach steht, können die Spielerinnen und Spieler hunderte von Stunden damit verbringen, noch die entlegensten Ecken von Tamriels Nordprovinz zu erkunden; zahllose Häuser, Dungeons, Festungen, Türme und Schiffswracks warten darauf, dass sich verwegene Abenteurer (mehr oder weniger legal) Zutritt zu ihnen verschaffen.

Nichts von all dem gibt es in THE BANNER SAGA. Stattdessen vollzieht sich das ganze Spiel - von ein paar wenigen filmischen Szenen abgesehen - im Rückgriff auf eine Handvoll Bildtypen, die einander abwechseln. Es gibt die Bilder von dem Lager, das man unterwegs aufschlagen kann, beziehungsweise von ausgewählten Orten, die man im Verlauf des Marsches erreicht: zumeist mehr oder weniger elende Dörfer und Städtchen. Auf diesen Bildern lassen sich einzelne, besonders markierte Lokalitäten anklicken, und mitunter auch bestimmte Figuren, was dann weitere Handlungsoptionen eröffnet: Beispielsweise erlaubt es ein Besuch des Marktes, unter anderem Vorräte für die Reise zu kaufen. Dann gibt es die Darstellung von Gesprächssituationen, die die Figuren in halbnahen Einstellungen zeigen und mehr oder weniger nach dem Schuss-Gegenschuss-Prinzip organisiert sind, also verschiedene Perspektiven in einem homogenen Dialograum zusammenfügen. Wobei das, was etwa Rook oder Alette sagen, wohlgemerkt nicht vertont wird: Stattdessen lesen wir die einzelnen Repliken und klicken die von uns bevorzugte Entgegnung oder Reaktion an (die mitunter darin bestehen kann, zu schweigen). Des Weiteren gibt es Bilder, die die Karawanen während ihres Marsches zeigen: Von links nach rechts oder von rechts nach links scrollt das Bild, und wir erblicken verschwindend kleine Züge von Menschen, Varl wie THE BANNER SAGA die gehörnten Riesen nennt, die sich mehr oder weniger freiwillig mit ihnen verbündet haben - und vereinzelten Viehkarren, die unter einem langen, im Wind sich streckenden Banner durch die weite, einsame Winterwelt ziehen. Schließlich gibt es die eingangs erwähnte Karte, die sich jederzeit aufrufen lässt; hier erkennt man - anhand eines Ikons, welches das Gesicht des jeweiligen Anführers zeigt (oder, ab dem zweiten Teil, möglicherweise der Anführerin) -, wo man sich gerade befindet und kann wiederum bestimmte Örtlichkeiten anklicken (Städte, Gebirgszüge, Wälder, Sümpfe, Buchten, Inseln ...), 
woraufhin eine Texttafel erscheint, die einiges an Hintergrundwissen $\mathrm{zu}$ der entsprechenden Lokalität bereithält. ${ }^{155}$ Und dann gibt es natürlich die rundentaktischen Kämpfe: Von schräg oben erblickt man isometrisch gestaltete Schlachtfelder, auf denen sich Menschen, Varl und Dredge gegenüberstehen (und späterhin obendrein die zentaurenhaften Horseborn sowie andere, weniger humanoide Kreaturen ${ }^{156}$ ); Zug um Zug wird hier gekämpft, wobei jede Figur über bestimmte Eigenschaften, Stärken und Schwächen verfügt, sodass sich die Spieler sehr gut überlegen müssen, wer in welcher Reihenfolge was tun soll.

Von den isometrischen Schlachtfeldern und namentlich den Kämpfern beider Seiten abgesehen, sind all diese Bildtypen spärlich oder gar nicht animiert in den Gesprächssituationen verfügen die Figuren etwa nur über minimale Gestik oder Mimik; jemand blinzelt, Finger trappeln auf dem Rand eines Schildes, Augenbrauen werden hochgezogen - und bieten den Spielern und Spielerinnen wenige und vor allem eindeutig festgelegte Handlungsoptionen. Während wir den Zug der Karawanen beobachten, steht es uns beispielsweise nicht frei, das Marschtempo oder die Route festzulegen. Wir können zwar jederzeit ein Lager aufschlagen lassen, das bringt aber nur etwas, wenn wir die Karawane einen Tag pausieren lassen und über genügend Nahrungsmittel verfügen, damit sich die erschöpften Wanderer während der Rast stärken können. Ansonsten bleibt uns wenig übrig, als der Reise durch die kalte Einsamkeit zuzuschauen, zu beobachten, wie die Vorräte schwinden, die Stimmung immer schlechter wird - und zu warten, bis der nächste Zielpunkt erreicht ist, oder THE BANNER SAGA ein Textfenster öffnet, um ein Zufallsereignis anzukündigen, das häufig genug (wenn etwa ein jäher Schneesturm aufzieht oder eine Gruppe halbverhungerter Fremder um Nahrung bittet) erneut zum Verlust von Vorräten oder Zuversicht (oder beidem) führen wird.

Also noch einmal: Ist es am Platze, von einer Spielwelt zu sprechen, in Anbetracht der Art und Weise, wie THE BANNER SAGA die Spielerinnen und Spieler an kurzer Leine durch verschiedene streng reglementierte Bildtypen führt? Und kann man sagen, dass die Spieler hier einen Genuss an der Exploration der

155 Das Beispiel von tHE BANNER SAGA legt nahe, dass sich die Ergebnisse, die Stefan Ekmans Lektüre von Karten aus Fantasy- beziehungsweise Fantastik-Romanen hervorgebracht hat, auf andere Medien als das Buch übertragen lassen: „More surprisingly, the topofocal readings revealed much about fundamental aspects of the works, such as their underlying attitudes and central concerns. These readings turned out to be useful in clarifying the roles and nature of certain characters, and they helped demonstrate how plot, character, and setting are interwoven.“ Ekman: Here be Dragons, S. 219.

156 Im dritten Teil der BANNER SAGA, wenn man schließlich in die erwähnte metaphysische Dunkelheit vorrückt, kommen noch dämonisch verwandelte Variationen der Bewohner der Spielwelt hinzu. 
Spielwelt finden, gar gestalterisch auf die Rhythmik, Zeitlichkeit und Moralität dieser Welt einwirken, wenn sie sich nicht einmal eigenständig bewegen dürfen?

Die erste dieser Fragen ist leicht zu beantworten. So, wie die Diskussion in der Fachforschung geführt wird, gibt es keinerlei Grund, davon auszugehen, dass eine Spielwelt, um als solche gelten zu dürfen, über dreidimensionale Grafik verfügen oder den Spielerinnen und Spielern die Freiheit der Erkundung gewähren müsste. ${ }^{157}$

Nach der Definition von Mark J. P. Wolf könnte man sogar sagen, dass die „Welt“ im Videospiel eine grundlegende Funktionalität des Mediums bezeichnet, die völlig losgelöst von jedweder sinnlichen Konkretion besteht:

As the action of most video games takes place in a virtual space over time and features some sort of causality, the settings in which games take place are often referred to as "game worlds", and as such, they have a place in the history of imaginary worlds. Video game worlds are necessarily composed of several things: some kind of geography, inhabitants, action, and logical consequences that are the outcome of actions. Every game world has some kind of space in which the game's action takes place, from simple blank playing fields that are a single screen in size (as in many early arcade games), or a verbal description (in the case of text adventures), to vast, elaborately detailed worlds with hundreds of thousands of players (as in massively multiplayer online role-playing games (MMORPGs)). These areas are displayed on-screen, and many games, especially adventure games, require exploration of the game world, where other characters are encountered, objects are found, and quests are completed..$^{158}$

Vielleicht ist Wolf diese Definition ein bisschen zu weit geraten, da sie - auf theoretischer ebenso wie auf poetologischer Ebene - eigentlich keine Binnendifferenzierung zwischen „simple blank playing fields“ und „vast, elaborately detailed worlds“ erlaubt. In ihrer Allgemeinheit haben seine Bestimmungen jedoch einen großen Vorteil, insofern sie nämlich unzweifelhaft deutlich machen, dass die Spielerinnen und Spieler einen digitalen Raum keineswegs nur dann als „Welt“ erleben, wenn dieser Raum in irgendeiner Form repräsentational auf die Alltagswelt bezogen werden kann.

Wie aber wird ein Spielraum zur Spielwelt in einem engeren Sinn? Ich denke, die folgenden Ausführungen geben eine Antwort auf diese Frage:

157 Marc Bonner etwa stellt rein deskriptiv fest, dass die Welten der Videospiele „heute meist dreidimensional“ ausgestaltet sind, ohne daraus irgendeine normative Festsetzung abzuleiten. Vgl. Bonner: Welt, S. 129.

158 Mark J. P. Wolf: Worlds. In: ders./Perron (Hg.): The Routledge Companion to Video Game Studies, S. 125-131, hier: S. 125. 
[...] a game world will operate according to some logic that it uses to assign consequences to actions taken by the game world's characters. These consequences usually are consistent and can be expected in advance once the player learns how the world works. Through knowledge of these consequences, players can make gameplay choices that move the game world's state in a desired direction. The game world's logic determines much of the gameplay experience, and may also shape the look and feel of the game world itself, suggesting guidelines for design aesthetics. ${ }^{159}$

Wolfs Überlegungen lassen sich ohne Weiteres rückbinden an die Begriffe, die ich auf den vorangegangenen Seiten verwendet habe. Man könnte also sagen: Die Spielwelt wird zur Welt, insoweit sie sich den Spielerinnen und Spielern als ein Raum darbietet, dessen Gesetzmäßigkeiten im Spielprozess erfahren, ergründet und reflektiert werden können - sodass also eine Art poetologische Kommunikation zwischen Spieler und Spielwelt entsteht, eine wechselseitige Bezugnahme in Aktion und Reaktion, wobei aus der Perspektive der Spielerfahrung keineswegs immer klar ist, wem das Handlungsprimat zukommt. So gesehen verfügen sehr viele, aber nicht alle Spiele über „Welten“, da es ja - zum Beispiel bei Sportspielen - durchaus möglich ist, dass ein Raum vorwiegend als Kulisse, Hintergrund oder Staffage fungiert.

In einem, wie mir scheint, durchaus vergleichbaren Sinn schlägt Stephan Günzel vor, Videospiele „als bereits ,gelebte‘ Räume“ zu verstehen, „insofern sie das Resultat eines Prozesses sind, bei dem bestimmte Weltvorstellungen sich mit einer Praxis des Spielens verschränken, die von Regelsystemen bereitgestellt werden““. ${ }^{160}$ Und er erläutert, dass diese Betrachtungsweise ,eine neue philosophische Sichtweise auf Computerspiele“ eröffne, „nämlich: sie selbst als Elemente einer Menge zu verstehen, die durch sie exemplifiziert werden“. ${ }^{161}$

Günzel führt aus:

So repräsentieren Egoshooter freilich den Raum (auch) als ,Schachtel', aber das ist kein Ausdruck einer ontologischen Minderwertigkeit, sondern die spezifische Darstellungsleistung. Computerspiele machen solcherart Raumkonzepte erfahrbar, die anderweitig nur über theoretische Konstrukte [...] zugänglich sind. ${ }^{162}$

Auf der Grundlage dieser Ansätze sollte es nun möglich sein, das „Raumkonzept“ zu bestimmen, welches als ästhetische Idee die Weltgestaltung von THE BANNER SAGA anleitet - und also eine Antwort auf die Frage zu geben, wie sich

159 Wolf: Worlds, S. 126.

160 Stephan Günzel: Raum. In: Feige/Ostritsch/Rautzenberg (Hg.): Philosophie des Computerspiels, S. 221-240, hier: S. 235.

161 Günzel: Raum, S. 235.

162 Günzel: Raum, S. 235. 
dieses Spiel das Verhältnis zwischen Spieler und Spielwelt denkt. Ich möchte mich hierzu auf einen Vorschlag von Thomas Hensel beziehen, der John L. Austins Sprechakt-Theorie auf das Medium Videospiel zu übertragen sucht. ${ }^{163}$ Das „Computerbild“ gilt Hensel als „sichtbare Manifestation eines digitalen, operativen Codes, der die Trennung von Ausführung (Aktion) und Darstellung (Repräsentation) unterläuft“. ${ }^{164}$ Das heißt: „Man vollzieht etwas im Gebrauch dieser Bilder, die sich damit als Bildakte erweisen.“ Und weiter: „Wie das Computerbild ist auch das Computerspielbild ein Bildakt (= Performativität erster Ordnung), sozusagen ein momenthaft erspieltes Bild, das nur im Augenblick seines Vollzugs existiert."165 Hensel geht noch einen Schritt weiter, und schreibt dem Videospiel (oder Computerspiel) die Möglichkeit zu, sich als „doppelter Bildakt“ zu realisieren; dies geschieht stets dann, wenn Bildakte „auf der Oberfläche des Computerspiels“ auftreten, wenn dieses „also selbstreflexiv oder opak wird, sprich seine Bildlichkeit als eine seiner Bedingungen bildlich thematisiert. “166

Er erläutert:

Das Computerspiel zeichnet sich auf diese Weise nicht nur durch seine Narrativität oder Ludizität aus, sondern auch und gerade durch seine Ikonizität, die jene anderen Eigenschaften von Fall zu Fall in sich zu integrieren vermag. Es gilt somit, das Bild nicht nur als eine Funktion des Narrativen oder Ludischen zu verstehen, sondern umgekehrt das Narrative oder Ludische auch als eine Funktion des Bildes. Eine Pointe dieses Ansatzes ist es, dass das Medium Bild die Spielherausforderungen entgegen dem gängigen Klischee nicht nur konturiert und kontextualisiert, sprich rahmt, sondern vielmehr das Bild selbst die Spielherausforderung ist. Und mehr noch: Nicht nur wird das Bild gespielt das Bild spielt auch. ${ }^{167}$

Es scheint mir vielversprechend, THE BANNER SAGA in dieser Perspektive zu betrachten. Das Spiel von Stoic Studio gestaltet fortwährend doppelte Bildakte zumindest dann, wenn man Hensels Überlegungen nicht nur auf das einzelne Bild bezieht, sondern desgleichen auf den Erfahrungsmodus, den die audiovisuelle Bildlichkeit eines Spiels in ihrer Gesamtheit hervorbringt -, insofern es nach allen Regeln der Kunst (beziehungsweise der im ersten Kapitel entwickelten Poetik) eine Fantasy-Welt ins Werk setzt, die Möglichkeiten, mit dieser Welt $\mathrm{zu}$ interagieren, jedoch zugleich entschieden reduziert. THE BANNER SAGA „spielt“ also gleichsam mit den Wünschen der Spielerinnen und Spieler, mit

163 Vgl. John L. Austin: How to do Things with Words. Cambridge, Mass. 1962.

164 Thomas Hensel: Bild. In: Beil/Hensel/Rauscher (Hg.): Game Studies, S. 47-62, hier: S. 56.

165 Hensel: Bild, S. 56 [Herv. i. O.].

166 Hensel: Bild, S. 58.

167 Hensel: Bild, S. 58. 
ihrem Verlangen danach, sich einzusenken in die Welt des Spiels, ihre Geschichte ebenso wie die unzähligen, in ihren Weiten verborgenen Geschichten zu ergründen. Doch das, was wir faktisch tun können, läuft oft genug auf EntwederOder-Entscheidungen heraus, die als Form der Bezugnahme an sich schon die Distanzierung des Spielers verlangen, in ihrer konkreten Ausgestaltung aber immer wieder harsche und obendrein vergebliche Kalkulationen verlangen, die geradezu eine lieblose Haltung von den Spielerinnen und Spielern zu erzwingen suchen. Denn was THE BANNER SAGA von uns wissen will, läuft strukturell immer wieder auf die Frage hinaus, ob wir einen friedlichen Bauern einschüchtern und bedrohen wollen, um seines Viehs habhaft zu werden, oder Gerechtigkeit walten lassen - und damit riskieren, dass unsere eigenen Leute verhungern.

Das ist die eine Seite von THE BANNER SAGA; die andere Seite hat etwas damit zu tun, dass die Entwickler von Stoic Games ein bezaubernd schönes Spiel gestaltet haben. Dabei haben sie sich offenbar von dem Stil Eyvind Earles inspirieren lassen, der in den fünfziger Jahren als Zeichner für Disney gearbeitet und unter anderem an PETER PAN (Clyde Geronimi/Wilfred Jackson/Hamilton Luske, USA 1953) und SLEEPING BEAUTY (Clyde Geronimi/Les Clark/Eric Larson/Wolfgang Reitherman, USA 1959) mitgewirkt hat. ${ }^{168}$ Das Ergebnis ist ein ,edler Zeichenstrickstil‘ ${ }^{169}$ der manch einem ,die Augen übergehen' lässt. ${ }^{170}$

Die Spielerfahrung von THE BANNER SAGA lässt solcherart zwei Gestaltungsprinzipien kollidieren, die in jeweils gegenläufigen affektpoetischen Bewegungen sich ausdrücken: Die Augen gehen über, wollen sozusagen hineinfliegen in die melancholische Poesie einer dem Untergang geweihten Winterwelt, und das Herz gleich mitziehen; doch das unerbittliche Gesetz, das ebenjener Welt eingeformt ist, macht immer wieder eine Ent-Äußerung der Spielerinnen und Spieler erforderlich; sie müssen auf ihren Wunsch verzichten, sich der Welt von THE BANNER SAGA in träumerischer Sehnsucht zu überlassen, weil das Spiel ihnen auferlegt, mit und gegen diese Welt zu kämpfen, Spielminute für Spielminute, in immer neuen unmöglichen und unumgänglichen Entscheidungen. ${ }^{171}$

168 Vgl. Harman: THE BANNER SAGA review.

169 Jörg Luibl: Zwischen Verlust und Verantwortung. Test: THE BANNER SAGA 2. In: www.4play ers.de, 28. April 2016, https://www.4players.de/4players.php/dispbericht/Allgemein/Test/ 36238/81809/0/The_Banner_Saga_2.html [letzter Zugriff: 12.05.2020].

170 Alexander Bohn-Elias: THE BANNER SAGA-Test. Neulich im Wörterbuch unter „episch“. In: www.eurogamer.de, 17. Januar 2014, https://www.eurogamer.de/articles/2014-01-17-the-ban ner-saga-test [letzter Zugriff: 12.05.2020].

171 Bis zu einem gewissen Grad ähnelt die Weltkonzeption von THE BANNER SAGA hierin, wie wir sehen werden, jener von SHADOW OF THE COLOSSUS. 
Man kann also durchaus sagen, dass in THE BANNER SAGA eine Gestaltung der Spielwelt durch den Spieler stattfindet; nur geschieht das auf sehr besondere Weise, man muss sie dem Spiel nämlich abtrotzen, jene Spiel-Räume der Freiheit und Verzauberung. Das gilt in jeder Hinsicht. Ja, auch in THE BANNER SAGA bestimmt man (mindestens ein Stück weit) die Moralität, die der Spielwelt in ihrer Entfaltung zukommt. Aber man tut es in einem Prozess fortlaufenden Scheiterns, der darauf abzielt, eine Möglichkeit des Guten zu schaffen, die in der Welt eigentlich nicht - oder nicht mehr - vorgesehen ist. Oder aber in die Kapitulation der Spielerinnen und Spieler mündet. All die kleinen und großen Entscheidungen, die wir treffen müssen, summieren sich $\mathrm{zu}$ einer Grundentscheidung, die zwar, da es sich bei THE BANNER SAGA schließlich immer noch um ein Spiel handelt, verhältnismäßig leicht revidierbar ist, die Spielerfahrung aber nichtsdestoweniger mit großem Ernst erfüllt: Willst du jemand sein, so fragt uns das Spiel, der Fremden, die in Not geraten sind, zu helfen sucht - und dabei riskiert, enttäuscht und betrogen zu werden? Oder ziehst du dich zurück in die Verhärtung einer kalten und selbstsüchtigen Rationalität - die übrigens ihrerseits droht, an der Unwägbarkeit des eigenen Kalküls zu scheitern ${ }^{172}$ Kurzum: Ob sie wollen oder nicht, die Spielerinnen und Spieler sind stets auch Agenten

172 Unter Bezugnahme auf die Überlegungen von Tobias Unterhuber und Marcel Schellong könnte man sagen, dass THE BANNER SAGA zu jenen Entscheidungsspielen zählt, die einen Bruch zumindest mit einem neoliberalen Ökonomismus vollziehen. Die beiden schreiben: „Damit kann Ökonomismus eben nicht mehr die Funktion einer handlungsleitenden Strategie übernehmen, da die präsentierten Möglichkeiten keinen höheren Pay-off versprechen. Erst hier scheint die Entscheidung zur Entscheidung von Gewicht, zur schwerwiegenden Entscheidung zu werden. Die Vorstellung der Alternativlosigkeit von ökonomischen Lösungswegen wird als Alternative oder gar als richtige Wahl nun weitgehend ausgeschlossen. Damit mögen Spiele des Decision Turns sich von neoliberalen Denkarten ablösen, aber ob sie sich auch vom ökonomischen Denken ablösen können, bleibt einstweilen ungewiss. Denn gibt es nicht vielleicht auch so etwas wie einen ,emotional pay-off'? Und wird das ,richtige Handeln' nicht einfach nur von der Ebene des Spiels auf die Ebene der Spielerin verlegt, die für sich immer noch eine Idee des richtigen Handelns kennt?“ Unterhuber/Schellong: Wovon wir sprechen, wenn wir vom Decision Turn sprechen, S. 22.

Allerdings würde ich behaupten, dass THE BANNER SAGA einiges daransetzt, vermeintliche Gewissheiten auf Seiten der Spielerinnen und Spieler, was eine Vorstellung vom „richtigen Handeln“ betrifft, zumindest zu erschüttern. Vgl. zu der Frage, inwieweit die Entscheidungen im Videospiel bezogen auf die Spieler eine gewissermaßen pädagogische Funktion haben können: Nina Köberer/Patrick Maisenhölder/Matthias Rath: Der homo narrans und ethisch-moralische Entscheidungssituationen in digitalen Spielen. In: Redaktion Paidia (Hg.): „I'll remember this“, S. 193-213, v. a. S. 201 u. S. 209 f. 
sowohl der Katastrophe als auch der Eukatastrophe, ${ }^{173}$ mal so, mal so, wiewohl in den seltensten Fällen klar ist, welche Konsequenzen ein bestimmtes Vorgehen nach sich zieht.

Und ja, man prägt auch die Zeitlichkeit der Spielwelt. Manches darf man tatsächlich entscheiden: Es gibt vermeidbare Kämpfe und Begegnungen, und wer genug Nahrungsmittel besitzt, kann immer wieder Rasten einlegen, um die Seinen zu schonen. Tatsächlich hält das Spiel vier Trophäen bereit, die sich unmittelbar auf die Möglichkeiten der Zeitgestaltung beziehen, die den Spielerinnen und Spielern gegeben sind: Man bekommt sie, wenn man besonders schnell marschiert oder besonders viele Schlachten schlägt - oder, wenn man dafür sorgt, dass niemand in der Karawane verhungert und die Moral nicht ins Bodenlose sinkt. Wohlgemerkt ist es unmöglich, diese Trophäen allesamt in einem Spieldurchgang zu erringen, weil jemand, der einen Gewaltmarsch initiiert, damit eben letztlich das Elend der eigenen Leute in Kauf nimmt.

Vor allem aber erfüllt sich im Explorationsdrang der Spielerinnen und Spieler eine bestimmte Zeitlichkeit. Wer in THE BANNER SAGA die Spielwelt erkunden will, tut dies einmal, indem er die Karte studiert; vor allem aber, indem er den Zug der Menschen und Varl mit wachen Augen begleitet, auf die Schönheit der Winterwelt achtet, sich an den Einzelheiten der Gestaltung erfreut, an den gewaltigen Göttersteinen, die, als monumentale und traurige Überbleibsel einer vergangenen Epoche, über die Spielwelt verstreut sind; an den Hügeln und Bergen, die sich fern im dunstigen Hintergrund erheben; an den schattenhaften Silhouetten von Bäumen und Gesträuch (und lauernden Dredge), die die Gestaltung des Bildvordergrunds prägen.

Aber, wie gesagt, all das muss man dem Spiel abringen. Und zweifellos setzt THE BANNER SAGA auch auf Spielerinnen und Spieler, die dergleichen zu tun versuchen. Dann nämlich gestaltet sich die Spielerfahrung als konflikthafte Auseinandersetzung mit dem Gesetz der Spielwelt. Es ist ein fortwährendes Aufbegehren gegen die Erbarmungslosigkeit dieser Welt, das im Wechsel mit der zähneknirschenden Anerkennung all der Überlebenszwänge, die das Spiel verhängt, einen ganz eigenen Rhythmus taktet.

173 Für den letzten Teil der Trilogie gilt diese Einschätzung freilich nur mit Abstrichen. Je nachdem, welche Entscheidungen die Spielerinnen und Spieler im dritten Akt treffen und wie gut sie sich in den rundentaktischen Kämpfen des Finales schlagen, kann die BANNER SAGA durchaus in einer Eukatastrophe enden, die im strengen Sinn dem entspricht, was sich Tolkien unter dem Begriff vorgestellt hat: d.h., der Welt von Rook und Alette erwachsen Hoffnung und Zukunft, auch wenn einzelne Figuren einen hohen Preis dafür zahlen, dass dieses Ende möglich wird. 
So werden sich die Spielerinnen und Spieler hinreichend an der Spielwelt aufgerieben haben, wenn das Finale von THE BANNER SAGA beginnt, dass die exemplarische Eukatastrophe, die dieses Finale gestaltet, sie mit aller Wucht zu berühren vermag. Exemplarisch ist die Eukastastrophe, mit welcher der erste Teil der Trilogie endet, nämlich sowohl in dem jähen Aufstrahlen einer unmöglichen Hoffnung, als auch in dem bitteren Schmerz, der den Schattenwurf jenes Lichtes bildet. Zwar gelingt es den Menschen und den Varl, gegen eine schier unüberwindliche Dredge-Armee zu bestehen und den Anführer der Steinwesen, den unsterblichen Bellower in die Knie zu zwingen, doch ist der Preis dieses Sieges das Leben von entweder Rook oder Alette. ${ }^{174}$ Vielleicht wissen das die Spielerinnen und Spieler sogar - nachdem sie im Verlauf von THE BANNER SAGA reichlich Lehrgeld haben zahlen müssen -, wenn sie die Entscheidung treffen, ob Vater oder Tochter gegen Bellower anzutreten hat.

Auf diese Weise gestaltet THE BANNER SAGA ein Konzept von Heldentum, das, als Spielerfahrung, eine große Ähnlichkeit aufweist mit der tragischen und ausweglosen Tapferkeit, die, folgt man Tolkien, auch Beowulf besingt. Es ist ein Heroismus der Ohnmacht; er verlangt, die Ohnmacht, das Scheitern und das Versagen auszuhalten. Immer wieder mit anzusehen, wie der Versuch, das Gute zu tun, etwas Schlechtes herbeiführt - und dennoch nicht aufzugeben. Sie nicht mitzumachen, die Verwandlung in eine Rechenmaschine, die nur noch ein Kalkül von Gewinn und Verlust kennt. Der Pessimismus, der dieser Idee von Heldentum eingeschrieben ist, rührt daher, dass eine Veränderung der Umstände, die das heroische Handeln notwendig machen, ausgeschlossen scheint. Die Regeln des Spiels, das heißt die Gesetzmäßigkeiten der Spielwelt, lassen sich nicht ändern - oder aber die Entstehung der Möglichkeit, sie zu ändern, ist an das Heraufdämmern einer Zukunft nach dem Ende der Trilogie gebunden. Was bleibt, ist der Eigen-Sinn, an Werten und Haltungen festzuhalten, obzwar sie die eigenen Gewinnchancen nicht erhöhen, sondern vielleicht sogar minder: ein Heroismus, den die Spieler etwa Rook und Alette angedeihen lassen können, wenn sie ihre eigene Haltung zu dem Spiel gefunden haben.

Zwar werden die Figuren von THE BANNER SAGA nur deshalb zu Helden, weil sie keine andere Wahl haben. Die meiste Zeit sind sie auf der Flucht und sehen voll ratlosem Schrecken dabei zu, wie ihr Leben und ihre Welt in Stücke brechen. Und die allerwenigsten von ihnen kämpfen, weil sie sich stark und

174 Bellower ist übrigens tatsächlich unsterblich; in einer merkwürdigen Volte zielt das Finale von THE BANNER SAGA darauf, dem Dredge-Krieger mithilfe eines magischen Pfeils vorzugaukeln, dass er gestorben sei - merkwürdig ist diese Volte vor allem, wenn man Bellowers Schein-Tod mit dem sehr endgültigen und, wenn man so sagen kann, poetologisch realen Tod von Rook oder Alette kontrastiert. 
mächtig fühlen. Doch kämpfen tun sie. Irgendwann kommt - und das ist in der BANNER-SAGA-Trilogie eben wörtlich zu nehmen - eine allesverschlingende Dunkelheit, die die Herdfeuer zum Erlöschen und die Lieder zum Verstummen bringt. Die Heldinnen und Helden des Spiels stellen sich dieser Dunkelheit entgegen. Es ist nicht so, dass sie an den Sieg glauben. Aber sie fügen sich auch nicht in die Niederlage. Denn in ihnen stößt, wie es über einen alten, todkranken Mann heißt, der Rook und Alette in seinem unwahrscheinlichen Heroismus verschwistert ist, „ein unbändiger Trotz hoch, in dieser Welt zu bestehen und für eine andere, bessere, zu kämpfen“. ${ }^{175}$

\section{Polare Positionen der Spielerfahrung - Resümee und Ausblick}

Vermutlich könnte man mit einigem Recht sagen, dass SKYRIM und THE BANNER SAGA polare Positionen der Spielerfahrung markieren, wenn es um das Verhältnis zwischen Spieler und Spielwelt geht. SKYRIM lässt den Spielerinnen und Spielern stets die Wahl zwischen einer Fülle von verschiedenen Möglichkeiten; das betrifft die Welterkundung ebenso wie die Entwicklung des Avatars und die Moralität seines Handelns. Die Entscheidungen, die man hier zu treffen hat, sind selten unwiderruflich, schließen kaum je andere Seinsmöglichkeiten aus. Es gibt Ausnahmen: Beispielsweise kann man nicht gleichzeitig für die Stormcloaks und die Imperial Legion kämpfen. Aber das Verwerfungspotenzial, das in solchen Exklusionen zum Tragen kommt, ist ziemlich gering. Noch das Folgenreichste bleibt in SKYRIM seltsam folgenlos; das Spiel perfektioniert die Kunst, den Pelz zu waschen, ohne ihn nass zu machen. Mit dieser Feststellung ist indessen keine Kritik an Bethesdas Weltentwurf verbunden. Die ELDER SCROLLS-Reihe verwirklicht, ungeachtet alles Düsteren und Blutigen, den kindlichen Wunsch nach der FantasyWelt als einem Abenteuerspielplatz mit schier unendlich vielen Klettergerüsten.

Hingegen gibt sich THE BANNER SAGA alle Mühe, den Spielerinnen und Spielern von Anfang an klarzumachen, dass Wasser eine ziemlich feuchte und obendrein oftmals kalte Angelegenheit ist. Wir haben es hier mit einem Spiel zu tun, das nicht einfach, wie vielleicht alle Spiele, auf der Ebene des Programmcodes aus Entscheidungen gemacht ist, sondern sich in der Spielerfahrung als einzige Folge von harten, folgenschweren und oftmals eigentlich unentscheidbaren Entscheidungen darstellt. Während SKYRIM den Modus des Sowohl-als-auch perfektioniert, zielt THE BANNER SAGA auf ein glasklares Entweder-Oder. Entweder gebe

175 Friedrich Dürrenmatt: Der Richter und sein Henker/Der Verdacht [1951/52]. Zürich 1998, S. 164 . 
ich diese oder jene Antwort; entweder ziehe ich meine Bogenschützin auf dieses oder auf jenes Feld; entweder nutze ich meinen „Renown“ (die einzige Währung des Spiels) um Vorräte zu kaufen oder um die Fähigkeiten eines meiner Helden weiterzuentwickeln; entweder raste ich, damit mein verwundeter Varl-Krieger wieder zu Kräften kommt, und riskiere, dass mir vor der nächsten Stadt die Nahrungsmittel ausgehen, oder ich setze den Marsch fort und finde mich vielleicht in einem Kampf wieder, wo mein Varl gerade dann fehlt, wenn er am Nötigsten gebraucht wird.

Noch der Umstand, dass der Zug der Karawanen, im Spielerleben und der grafischen Konfiguration, immer nur in eine Richtung führt, trägt zur Herausbildung einer Zeitlichkeit bei, die das Entweder-Oder als unerbittliche Serialität unwiderruflicher Entscheidungen realisiert. Ob diese Entscheidungen tatsächlich, was den Handlungsverlauf und das Schicksal einzelner Figuren betrifft, in jedem Fall so weitreichende Folgen haben, wie THE BANNER SAGA suggeriert, ist dabei zweitrangig: Sie haben ihre Wahrheit und Wirklichkeit in der Erfahrung der Spielerinnen und Spieler. ${ }^{176}$ Dazu passt es denn auch, dass die Erkundung der mythopoetischen Welt, der Genuss ihrer zeitlichen und räumlichen Weiten, die Gestaltung ihrer Zeitlichkeit und Moralität bis zu einem gewissen Grad, wie ich zu zeigen versucht habe, gegen das Spiel, im Widerstand gegen seinen Weltentwurf, sich vollziehen müssen.

In diesem Sinn also bezeichnen SKYRIM und THE BANNER SAGA polare Positionen der Spielerfahrung. Vielleicht entsprechen diese Positionen spezifischen Formen des Spielgenusses. Sie mögen eine Ähnlichkeit aufweisen zu den von Christopher Goetz so bezeichneten Modi der „tether fantasy“ und der „accretions fantasy““. ${ }^{177}$ Goetz betont, dass er nicht etwa ein literarisches Genre im Blick

176 Ich stimme Tobias Unterhuber und Marcel Schellong zu, wenn sie schreiben, die Kritik, in Videospielen seien Entscheidungsmöglichkeiten „nur Illusion“, würde zwei „wichtige Dinge“ übersehen: „Erstens macht es sehr wohl einen Unterschied, wie die Spielerin sich entscheidet, egal wie klein die Entscheidung ist, da ihre Entscheidung durch die Handlungen ihrer Spielfigur Teil der Narration wird. Ob sie nun ,Hey` oder ,Hallo‘ wählt, macht mindestens den Unterschied, dass sie ,Hey“ oder ,Hallo“ sagt. Prozessual geht jede dieser Entscheidungen in die Erzählung ein und verändert sie und das Spielerleben und - wie Jody Macgregor anmerkt auch den Ton der Erzählung. Zweitens ist es nicht so wichtig, ob die Entscheidungen Auswirkungen haben, sondern dass die Spielerin annimmt, sie hätten welche. Die Inszenierung der Entscheidung macht die Momente der Entscheidung aus. Wir nehmen sie anders wahr. Wenn wir den Entscheidungsmomenten im heiligen Ernst des Spiels entgegentreten, werden sie erlebbar als Simulationen von realer Erfahrung. “ Unterhuber/Schellong: Wovon wir sprechen, wenn wir vom Decision Turn sprechen, S. 26.

177 Vgl. Christopher Goetz: Tether and Accretions. Fantasy as Form in Videogames. In: Games and Culture 7 (2012), H. 6, S. 419-440. 
hat, wenn er von „fantasy“ spricht, sondern vielmehr abhebt auf „a dynamic psychological concept related to play“178 - und er führt aus:

Let me provisionally define a tether fantasy as the pleasurable process of oscillating between feeling safe and feeling exposed, dwelling on the boundaries that separate the two. In turn, an accretions fantasy can be defined as the pleasurable process of correcting a weak or vulnerable body by accruing objects from the world of gameplay. ${ }^{179}$

Nun geht es Goetz weder um SKYRIM noch um THE BANNER SAGA - und genaugenommen, wie er ja selbst hervorhebt, überhaupt nicht um Fantasy-Spiele und Welten -, sondern um MINECRAFT (Mojang, 2009/2011) und TERRARIA (Re-Logic, 2011). Insofern wäre es vielleicht naheliegender, zunächst andere Bestimmungen heranzuziehen. Die in den Game Studies überaus beliebte, auf Roger Caillois' Spieltheorie zurückgehende Unterscheidung zwischen paidia und ludus bietet sich hier an. ${ }^{180}$ Im Anschluss an Caillois definiert Thomas Hensel paidia als das „freiere, unkontrollierte Spiel“; mit ludus hingegen ist für ihn das „stärker geregelte, eher zielgerichtete, mithin das disziplinierende Komplement von paidia“ bezeichnet. ${ }^{181}$ Sicherlich ist es unmittelbar einsichtig, wenn man SKYRIM in dieser Spanne auf paidia, THE BANNER SAGA hingegen auf ludus bezieht. Wichtig ist aber, dass Hensel zwischen paidia und ludus ein dialektisches Verhältnis ausmacht; wie er an THE LAST OF US (Naughty Dog, 2013) und LEFT BEHIND (Naughty Dog, 2014), der Erweiterung des Spiels, vorführt, lässt sich in dieser Dialektik durchaus eine (Selbst-)Reflexion des Mediums an sich erkennen. Für Hensel zeichnet sich die Dialektik von paidia und ludus unter anderem dadurch aus, dass in ihr „Geschichten blanken, aufreibenden Nah-

178 Goetz: Tether and Accretions, S. 419.

179 Goetz: Tether and Accretions, S. 419.

180 „An dem einen Ende herrscht fast uneingeschränkt das gemeinsame Prinzip des Vergnügens, der Ausgelassenheit, der freien Improvisation und der unbekümmerten Lebensfreude, wodurch sich eine gewisse unkontrollierte Stimmung, die man mit dem Namen paidia bezeichnen könnte, Ausdruck verschafft. Am anderen Ende wird diese fröhliche und impulsive Überschwänglichkeit durch eine komplementäre, ihrer anarchischen und launenhaften Natur in mancher Hinsicht zuwiderlaufende Tendenz fast völlig absorbiert bzw. diszipliniert: durch ein wachsendes Bedürfnis, sie willkürlichen, bindenden und absichtlich hemmenden Konventionen zu unterwerfen, sie durch die Errichtung zunehmend schwierigerer Hindernisse immer mehr zurückzudrängen und so den Weg zu dem ersehnten Ergebnis möglichst beschwerlich zu gestalten. Dieses Ergebnis ist ohne jeden Nutzen, obwohl es ein ständig zunehmendes Maß an Anstrengungen, Geduld, Geschicklichkeit oder Erfindungsgabe erfordert. Diese zweite Komponente nenne ich ludus.“ Roger Caillois: Die Spiele und die Menschen. Maske und Rausch [1958]. Berlin 2017, S. 34.

181 Thomas Hensel: Zwischen ludus und paidia. THE LAST OF US als Reflexion des Computerspiels. In: Beil/Freyermuth/Gotto (Hg.): New Game Plus, S. 145-183, hier: S. 154. 
kampfs und dessen Ästhetik“ mit ihrem „Gegenteil: Ruhe und Fernsicht“, konterkariert werden - und vice versa. ${ }^{182}$

Es scheint mir nicht unangemessen, aus Hensels Bestimmungen den Rückschluss zu ziehen, dass sich im Verhältnis von Spieler und Spielwelt eine Dynamik entfaltet, die die komplementären Konzepte paidia und ludus umschließt und in je eigenen poetologischen Entwürfen aufeinander bezieht. Wenn SKYRIM und THE BANNER SAGA polare Positionen der Spielerfahrung bezeichnen, so sind sie zugleich exemplarisch darin, dass sie in ihren Interpretationen dieser Dynamik auf die grundlegenden Möglichkeiten des Mediums Videospiel verweisen. Auch das ludische Oxymoron und der Modus der Fantasy sind Teil der so umrissenen Dynamik, insofern sie das Potenzial zu einer schier endlosen Kombinatorik in sich tragen, die weit über das hinausreicht, was man im engeren Sinn einer der Tradition Tolkiens verpflichteten Poetik zurechnen würde.

SKYRIM mag also eine besonders entschiedene Ausdeutung der ästhetischen Idee eines Open-World-Spiels (nicht nur) im Genre Fantasy darstellen, wohingegen THE BANNER SAGA in dem eigenwilligen Zeichentrickstil, der Mechanik der Karawanen-Verwaltung und vor allem dem gleichermaßen tieftraurigen wie heroischen, heillosen wie hoffnungsstarken Pathos unter den Videospielen wohl nur sich selbst entspricht. Zugleich jedoch vermessen beide Spiele die Möglichkeiten ihres Mediums, was Konzeptionen des Weltenbaus betrifft - womit ja zugleich ein je spezifisches Verhältnis zwischen Spieler und Spielwelt adressiert ist. Auch in den folgenden Spielanalysen, wenn es um SHADOW OF THE COLOSSUS oder DARK SOULS, um PYRE oder HELLBLADE: SENUA'S SACRIFICE geht, werden derartige Verhältnisse zu ergründen sein: als affektpoetische Kalkulationen, die den Genuss am Videospiel und der Fantasy ausmachen, die zugleich Entwürfe von Historizität, Gemeinschaft und Mensch-Sein (oder eben: Nicht-Mensch-Sein) realisieren.

Dabei sollte man nie vergessen, dass es nicht nur eine Dialektik zwischen paidia und ludus gibt, sondern desgleichen zwischen Erkunden und Verirren. In manchen Spielwelten, etwa jenen der DARK SOULS-Trilogie, kann man sich sehr leicht, sehr gründlich und durchaus buchstäblich verirren; bei anderen ist das strenggenommen nur möglich, wenn man darauf verzichtet, die Weltkarte zu Rate zu ziehen und (falls möglich) die sogenannten Questmarker abschaltet. Es gibt aber in nahezu allen Videospielen die Möglichkeit eines Verirrens, das nicht die räumliche Orientierungslosigkeit, sondern eine Form des ästhetischen Erlebens meint. Sie hat vorderhand mehr mit paidia als mit ludus zu tun, vollzieht sich aber ebenso inmitten der strengen Reglementierung eines rundentak-

182 Hensel: Zwischen ludus und paidia, S. 153. 
tischen Gefechts oder der disziplinierten (und überaus disziplinierenden) Rohstoffakkumulation von Spielen wie CONAN EXILES.

Ein solches Sich-Verirren tritt in Kraft, wenn man nicht mehr weiß, was genau das Auge sieht und das Ohr hört (beziehungsweise was das Ohr sieht und das Auge hört) und was die Hände tun (oder was mit ihnen getan wird). Dann ist es unwichtig, ob das Spiel spielt oder die Spielerinnen und Spieler. Sich verirrend, hat man einen Möglichkeitsraum betreten, der zwar sicherlich nicht grenzenlos, aber doch unerschöpflich ist. In ihm können wir etwas Neues denken und etwas Neues fühlen. Dieses Neue mag gar nicht viel mit dem zu tun haben, was uns das Spiel zu denken und zu fühlen geben will; dennoch hängt sein Entstehen davon ab, dass wir uns spielend auf die jeweiligen poetologischen Vorgaben einlassen. Es gibt also, meine ich, eine Schule des gezielten Sich-Verlaufens, die nicht nur eine Kunst des Lesens, sondern auch eine Kunst des Spielens bezeichnet. ${ }^{183}$

183 Vgl. Daniel Illger: Von der Kunst, sich zu verlaufen. In: Tolkien Times (2015/2016), S. 6. 
